Archive for

Organic Chemistry

Arkivoc 2020, part iv, 86-161

\title{
Hypervalent iodine-mediated synthesis and late-stage functionalization of heterocycles
}

\author{
Samata E. Shetgaonkar and Fateh V. Singh* \\ Chemistry Division, School of Advanced Science, Vellore Institute of Technology (VIT) Chennai, \\ Chennai-600 127, Tamil Nadu, India \\ Email: fatehveer.singh@vit.ac.in
}

\section{Dedicated to Prof Thomas Wirth}

Received 11-16-2020

Accepted 03-27-2021

Published on line $\quad 04-08-2021$

\section{Abstract}

Hypervalent iodine chemistry has witnessed exponential growth in organic synthesis in recent times. Because of the electrophilic and good-leaving nature of hypervalent iodine reagents, they react with different nucleophiles in various synthetic transformations such as rearrangements, $\alpha$-functionalization of carbonyl compounds, alkene difunctionalization and oxidation reactions. Importantly, the application of hypervalent iodine reagents in the construction of heterocycles is of great interest and has been well studied over the years. This review article highlights the recent developments accomplished by hypervalent iodine reagents in the synthesis and functionalization of heterocyclic compounds.

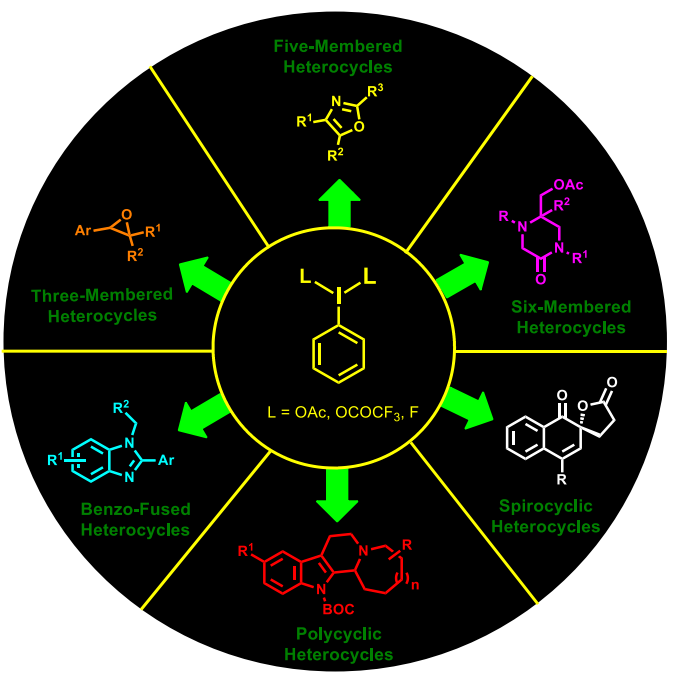

Keywords: Hypervalent iodine, late-stage functionalization, monocyclic, bicyclic, polycyclic, spirocyclic heterocycles 


\section{Table of Contents}

1. Introduction

2. Hypervalent lodine-Mediated Synthesis of Heterocycles

2.1. Synthesis of monocyclic heterocycles

2.1.1 Synthesis of three-membered heterocycles

2.1.1.1. Synthesis of aziridines

2.1.1.2. Synthesis of epoxides

2.1.2. Synthesis of five-membered heterocycles

2.1.2.1. Synthesis of oxazoles and oxazolines

2.1.2.2. Synthesis of isoxazole and isoxazolines

2.1.2.3. Synthesis of oxadiazoles

2.1.2.4. Synthesis of lactones

2.1.2.5. Synthesis of pyrrolidines, dihydropyrroles and pyrroles

2.1.2.6. Synthesis of pyrazolines, imidazolines and imidazoles

2.1.2.7. Synthesis of lactams and imidazolidinones

2.1.2.8. Synthesis of thiazoles and thiadiazoles

2.1.3. Synthesis of six- and seven-membered heterocycles

2.2. Synthesis of bicyclic heterocycles

2.3. Synthesis of polycyclic heterocycles

2.4. Synthesis of spirocyclic heterocycles

3. Hypervalent lodine-Mediated Late-Stage Functionalization of Heterocycles

3.1. Amination and azidation of heterocycles

3.2. Alkylation and alkynylation of heterocyles

3.3. Alkoxylation and acetoxylation of heterocycles

3.4. Halogenation and cyanation of heterocycles

3.5. Ring expansion of heterocycles

4. Conclusions

Acknowledgements

References

\section{Introduction}

Hypervalent iodine chemistry has become a focus of valuable research for designing robust methodologies in synthetic ${ }^{1}$ and natural product chemistry. ${ }^{2}$ Hypervalent iodine reagents are promising alternatives to the heavymetal oxidants due to their ready availability, easy handling, low toxicity and environmentally-benign nature. ${ }^{3}$ Synthetic applications of these reagents have seen exponential growth as realised by several books, ${ }^{4,5}$ chapters in books ${ }^{6,7}$ and comprehensive reviews ${ }^{8-11}$ published in this area. Both iodine(III) and iodine(V) compounds (also known as $\lambda^{3}$-iodane and $\lambda^{5}$-iodane) have been commonly used as reagents in the oxidative transformations of various simple and complex organic molecules. ${ }^{12-14}$ Most importantly, the unique reactivity and oxidizing ability of $\lambda^{3}$ - and $\lambda^{5}$-iodanes has prompted their use as efficient oxidants in variety of synthetic transformations including $\alpha$-functionalization of carbonyl compounds, ${ }^{15,16}$ oxidative rearrangements, ${ }^{17,18}$ alkene defunctionalization $^{19,20}$ and cyclization reactions. ${ }^{21,22}$ However, most of this transformation requires stoichiometric 
amounts of these reagents, generating the same molar quantity of iodoarenes as by-product thus limiting their scope. In order to combat this issue, several catalytic protocols including stereoselective variants have been developed extensively by employing various chiral/achiral iodoarenes as organocatalysts. ${ }^{6,23}$

Heterocycles constitute one of the widely studied classes of organic compounds as they are key to the structural units in various biologically and medicinally-important natural and synthetic products. ${ }^{24-26}$ Moreover, importance of heterocyclic compounds in material science 27,28 and pharmaceutical ${ }^{29}$ applications led their preparation and functionalization as topic of interest in organic synthesis. Owing to their wide scale importance, several researchers have devoted their studies in developing novel methodologies to access oxygen-, nitrogenand sulphur-containing heterocyclic scaffolds. Along these lines, hypervalent iodine chemistry has evolved as a powerful green strategy for their synthesis. Significant achievements have been accomplished in various hypervalent iodine-mediated or catalysed synthesis of heterocycles as discussed in the review articles by Sun et al., ${ }^{30}$ Kandimalla et al., ${ }^{31}$ and Singh et al. ${ }^{32}$ Further, late-stage functionalization constitute a powerful strategy for the manipulation of $\mathrm{X}-\mathrm{H}(\mathrm{X}=\mathrm{C}, \mathrm{N})$ bonds of a complex molecule into novel carbon-carbon and carbonheteroatom bond. ${ }^{33}$ Within this context, hypervalent iodine reagents have emerged as promising alternate candidates for transition metals in the direct $\mathrm{C}-\mathrm{H}$ functionalizations of diverse heterocycles via various synthetic transformations such as oxidative amination, alkylation, arylation, acetoxylation, halogenation, etc. ${ }^{33}$ This review article gives brief overview of the recent development of hypervalent iodine reagents in the synthesis and reactions of heterocyclic compounds. This review article is broadly divided into two categories i.e. synthesis of heterocycles and late-stage functionalization of heterocycles.

\section{Hypervalent lodine-Mediated Synthesis of Heterocycles}

Synthesis of heterocycles using hypervalent iodine reagents has seen dramatic progress in the last couple of decades. Having excellent electrophilic and oxidizing properties, iodine(III)/(V) compounds are choice of reagents to substitute toxic heavy metals. The use of these reagents in the construction of heterocyclic systems via $\mathrm{C}-\mathrm{C}, \mathrm{C}-\mathrm{O}, \mathrm{C}-\mathrm{N}, \mathrm{C}-\mathrm{S}, \mathrm{N}-\mathrm{N}, \mathrm{N}-\mathrm{S}$ or $\mathrm{N}-\mathrm{O}$ bond formation reactions have been well explored by several researchers. ${ }^{30-32}$ Most of these reactions employs iodine(III)/(V) reagents as stoichiometric oxidants. Apart from this, several catalytic systems involving in situ generation of hypervalent iodine species from aryl iodides in the presence of suitable terminal oxidant are well explored. Further this section is classified based on synthesis of monocyclic, bicyclic, polycyclic and spirocyclic heterocycles using different hypervalent iodine reagents.

\subsection{Synthesis of monocyclic heterocycles}

Various cyclization reactions employing hypervalent iodine reagents as oxidants are developed for the synthesis of monocyclic heterocycles in distinguish yields. Most of the approaches are free from metal catalyst while few require presence of copper or palladium species as catalysts. Moreover, enantioselective synthesis of heterocycles has been was achieved using chiral iodoarenes as precatalyst. In this section, hypervalent iodinemediated synthesis of three-, five-, six- and seven-membered heterocycles will be covered.

\subsubsection{Synthesis of three-membered heterocycles}

2.1.1.1. Synthesis of aziridines. In 2018, Jacobsen and co-workers demonstrated an elegant method for the diastereospecific synthesis of syn-6-fluoroaziridines $\mathbf{3}$ from cinnamylamine derivatives $\mathbf{1}$ using a catalytic amount of chiral aryl iodide 2 (Scheme 1). ${ }^{34}$ Reaction was hypothesized to proceed via trapping of key intermediate 4 by internal nitrogen nucleophile. The present fluoroaziridination reaction employs HF-pyridine 
as the nucleophilic fluoride source in the presence of stoichiometric oxidant mCPBA. Substrates with electronwithdrawing substituents yielded the desired products as single diastereoisomers with high enantioselectivity. Additionally, the scope of the reaction was extended to the synthesis of the five-membered heterocycle anti- 6 fluoropyrrolidine in $82 \%$ yield with ee up to $86 \%$.

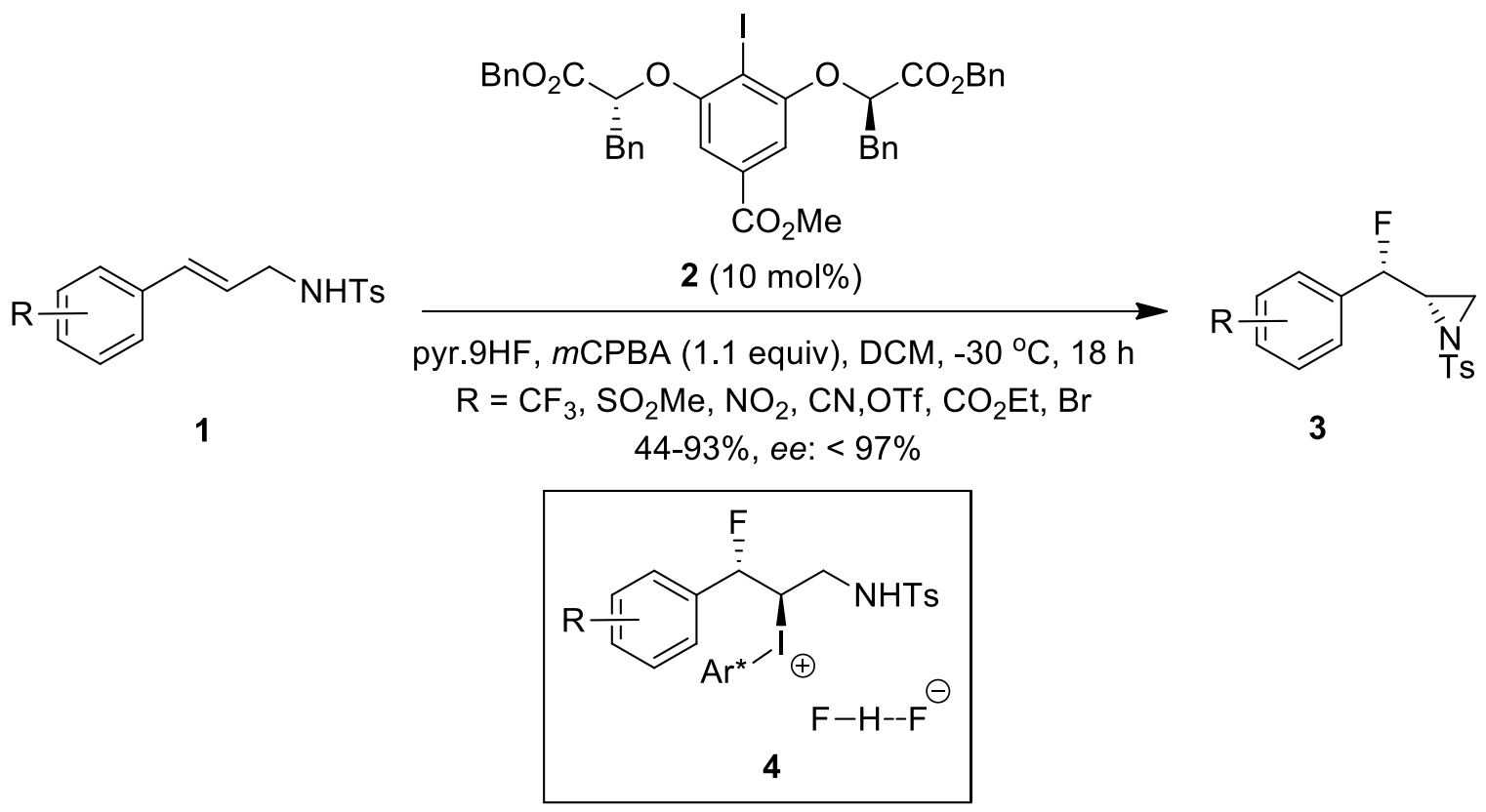

Scheme 1. Synthesis of syn-6-fluoroaziridines $\mathbf{3}$ using chiral iodide $\mathbf{2}$ as precatalyst.

Later, Reboul's team developed an unprecedented approach towards the synthesis of terminal diazirines 7 from amino acids 5 using ammonia as the nitrogen source (Scheme 2). ${ }^{35}$ This one-pot reaction involves PIDAmediated decarboxylation of amino acid $\mathbf{5}$ giving an imine intermediate, followed by insertion of the iodonitrene (formed in situ from the reaction of PIDA 6 and $\mathrm{NH}_{3}$ ) to form a diaziridine 8 and final oxidation to provide the desired diazirines 7. Several functional groups such as arene, heteroarene, ester, carboxylic acid, amide, sulfide, sulfoxide, etc. present in the amino acid side chain were well tolerated. Additionally, synthesis of terminal ${ }^{15} \mathrm{~N}_{2}-$ diazirines was achieved from unlabelled amino acids using ${ }^{15} \mathrm{NH}_{3}$ as a nitrogen source. Finally, hyperpolarization of ${ }^{15} \mathrm{~N}_{2}$-diazirine derivative was investigated using the SABRE-SHEATH method, demonstrating its potential application as hyperpolarized molecular tag.
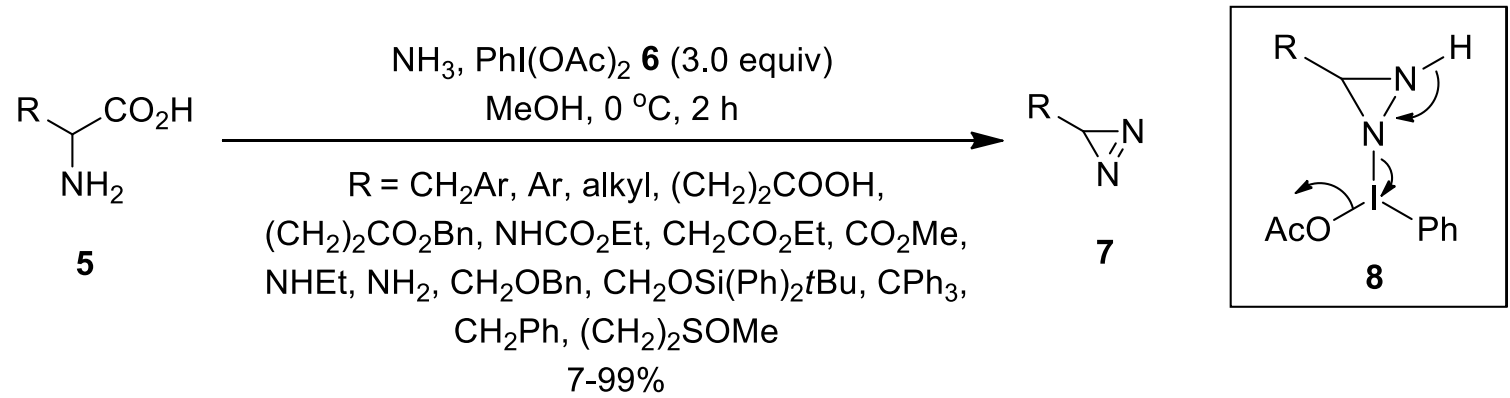

Scheme 2. Synthesis of terminal diazirines 5 using PIDA 6 as an oxidant. 
Very recently, Du and co-workers reported the synthesis of first novel hypervalent iodine reagent 10 bearing both iodine(III) and iodine(V) moieties through $m$ CPBA-mediated oxidation of $o$-nitroiodobenzene (Scheme 3). ${ }^{36}$ The synthesized iodine(III/V) compound $\mathbf{1 0}$ proved to be effective oxidant in preparing $2 \mathrm{H}$-azirines $\mathbf{1 1}$ from $\alpha$ substituted enamines 9 via intramolecular oxidative azirination process. Reaction tolerated variety of substituents at the ortho-, meta- or para-position of the phenyl ring affording the desired products in moderate to good yields.

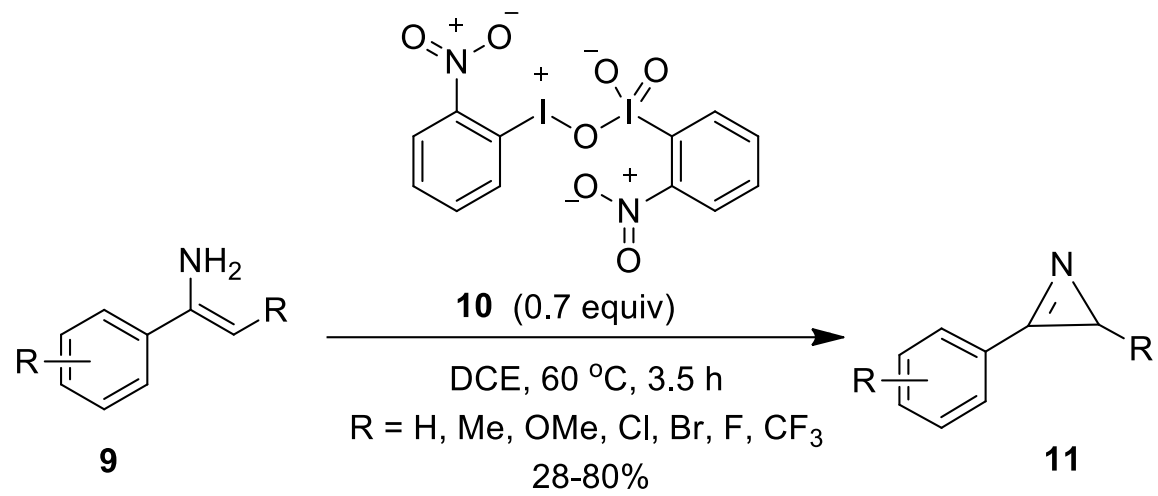

Scheme 3. Synthesis of $2 H$-azirines 11 using iodine(III/V) compound 10 as an oxidant.

2.1.1.2. Synthesis of epoxides. Mangaonkar and Singh developed a convenient ultrasound-assisted catalytic route to access $\beta$-cyanoepoxides 14 through epoxidation of $\beta$-cyanostyrenes 12 using iodobenzene 13 as precatalyst at room temperature (Scheme 4). ${ }^{37}$ The presence of oxone as terminal oxidant and TFA as an additive are crucial for the in situ generation of active iodine(III) species which reacts with alkene $\mathbf{1 2}$ and generates threemembered iodonium intermediate 15 followed by ring opening and cyclization to give anticipated product 14 . Reaction featured excellent functional group compatibility, high product yields and shorter reaction time. Previously, the same group also described epoxidation of $\beta$-cyanostyrenes with stoichiometric PIDA 6 under ultrasound irradiation conditions. ${ }^{38}$

$\mathrm{Phl} 13(10 \mathrm{~mol} \%)$

Oxone (2.0 equiv), TFA, $\mathrm{CHCl}_{3}, \mathrm{rt}, 60-90$

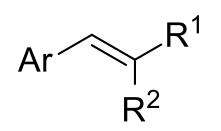

12

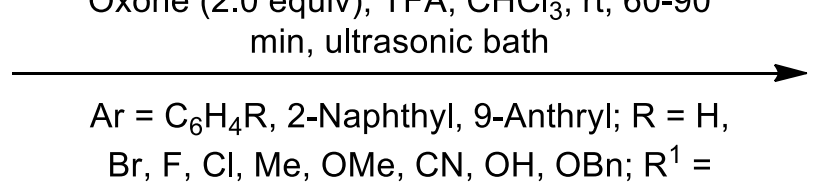

$\mathrm{CN}, \mathrm{CO}_{2} \mathrm{Et} ; \mathrm{R}^{2}=\mathrm{H}, \mathrm{CN}$ $65-94 \%$
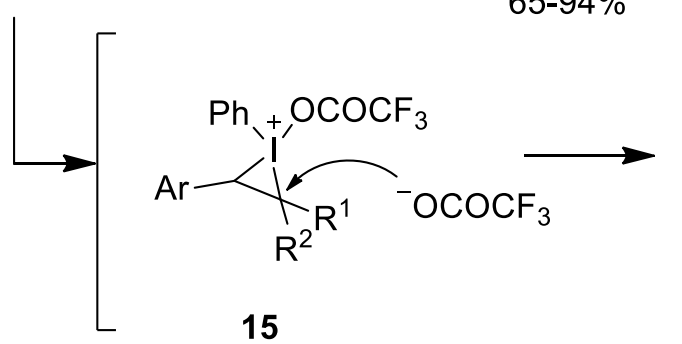

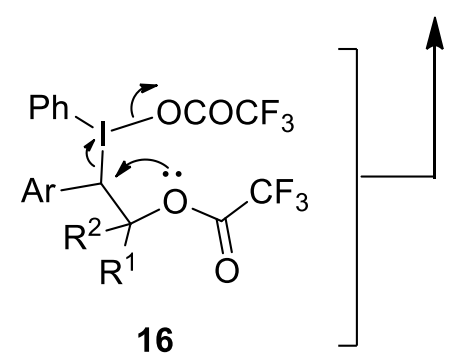

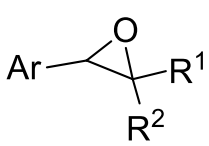

14

Scheme 4. lodine(III)-catalyzed epoxidation of $\beta$-cyanostyrenes 12 using iodobenzene 13 as precatalyst. 


\subsubsection{Synthesis of five-membered heterocycles.}

2.1.2.1. Synthesis of oxazoles and oxazolines. In 2016, Saito's research group reported synthesis of fluorinated oxazoles 20 through hypervalent iodine(III)-induced activation of N-propargyl amides 17 via a cycloisomerization-fluorination sequence (Scheme 5). ${ }^{39}$ This reaction occurs by employing either catalytic 4iodoanisole 18/HF-pyridine/Selectfluor system (Method A) or stoichiometric $p$-TollF 2 19/HF-Py system (Method B). Notably, stoichiometric method was found more effective for halogenated substrates. Later, the same group prepared 5-[( $N, N$-disulfonylamino)methyl]-oxazoles 22 by reacting $N$-propargyl carboxamides 17 with bisulfonyl(imides) 21 promoted by $\mathrm{PhI}(\mathrm{OAc})_{2} 6$ via cycloisomerization-amination sequence. ${ }^{40}$ The catalytic version of this method was developed using $\mathrm{Phl} 13$ as precatalyst with oxone as oxidant and $\mathrm{TBAHSO}_{4}$ as phase transfer reagent. Furthermore, Yi et al. ${ }^{41}$ combined iodocyclization and oxidative deiodination process for the conversion of $N$-propargylamides 17 into oxazole-5-carbaldehydes 23 using PIDA 6 (10 mol \%)/Lil/visible light system under oxygen atmosphere (Scheme 5).

Method A: 4-MeOC ${ }_{6} \mathrm{H}_{4} \mathrm{I} 18$ (20

mol\%), Selectfluor (2 equiv), HF.
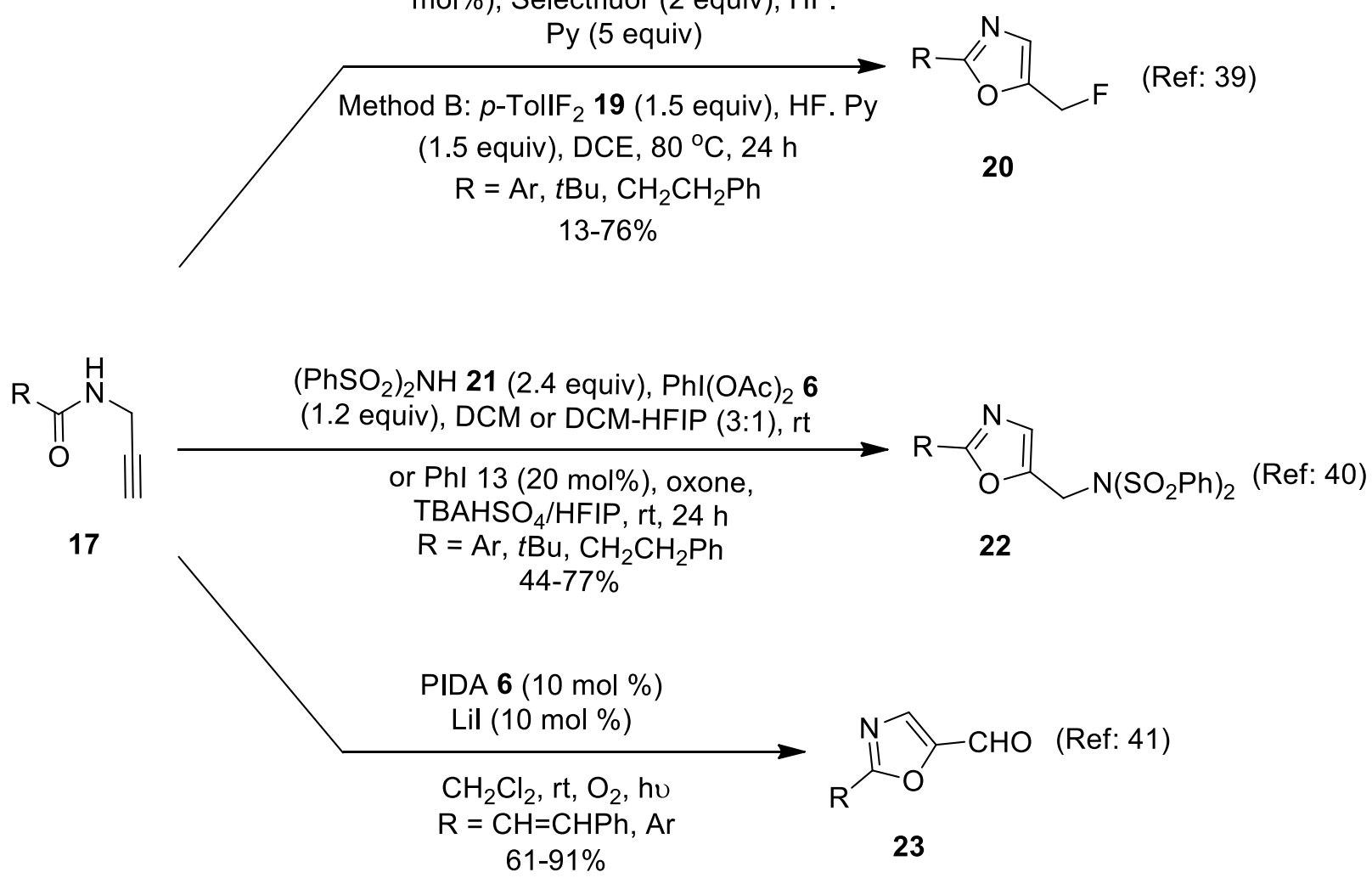

Scheme 5. Hypervalent iodine(III)-mediated synthesis of oxazoles 20, 22 and 23.

In continuation, Saito's research group reported [ $2+2+1]$ cycloaddition-type reaction of internal/terminal alkynes 24, nitriles 25 and oxygen atom from $\operatorname{Arl}(\mathrm{OH}) \mathrm{NTf}_{2}$, which is generated in situ from $\mathrm{Arl} / \mathrm{mCPBA} / \mathrm{Tf}_{2} \mathrm{NH}$ catalytic system (Scheme 6). ${ }^{42}$ This oxidative annulation represents the first example of iodine catalysis in multicomponent reactions enabling facile synthesis of 2,4-disubstituted and 2,4,5-trisubstituted oxazoles 28 . The reaction employed either $\mathrm{PhI} 13$ (Condition $\mathrm{A}$ ) or $4-\mathrm{ClC}_{6} \mathrm{H}_{4} \mathrm{l} 26$ (Condition $\mathrm{B}$ ) as precatalysts along with $m \mathrm{mCPA}$ and $\mathrm{Tf}_{2} \mathrm{NH}$. Additionally, reaction scope was also administered using iodosylbenzene 27 (1.8 equiv) and $\mathrm{Tf}_{2} \mathrm{NH}$ (Condition C). Notably, catalytic conditions A or B provided almost same results as stoichiometric condition $\mathbf{C}$. 
Further active iodine(III) species $\mathrm{Phl}(\mathrm{OH}) \mathrm{NTf}_{2}$ involved was isolated as an aquo-[18C6] complex in $43 \%$ yield under the optimized conditions.

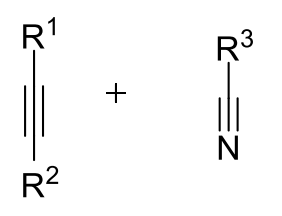

24
25

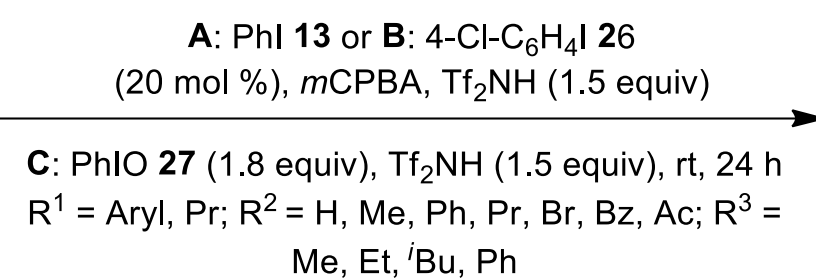

A: $24-75 \%$;<smiles>[R]c1nc([R])c([R])o1</smiles>

28

Scheme 6. lodine(III)-mediated synthesis of 2,4-disubstituted and 2,4,5-trisubstituted oxazoles 28.

Ding's group recently published a divergent protocol to access 2,5-disubstituted oxazole derivatives 30 via $\mathrm{Phl}(\mathrm{OAc})_{2}$-induced oxidative rearrangement of several allylic amides $29 .{ }^{43}$ Reaction was carried out in the presence of $\mathrm{BF}_{3} \cdot \mathrm{OEt}_{2}$ as an additive in $\mathrm{THF}$ at room temperature. Both aromatic and heteroaromatic substituents were well tolerated under optimized reaction conditions and products were obtained in variable yields (Scheme 7). Possible mechanistic approach initiates with the reaction of allylic amide 29 with Phl(OAc) 6 to form iodinated intermediate $\mathbf{3 1}$ which further gives intermediate 32. Next, nucleophilic attack by hydroxyl group at the carbocation of $\mathbf{3 2}$ generates cyclic intermediate $\mathbf{3 3}$ followed by subsequent aryl migration with the loss of Phl 13 gives species 35, which later gets converted into desired product $\mathbf{3 0 .}$
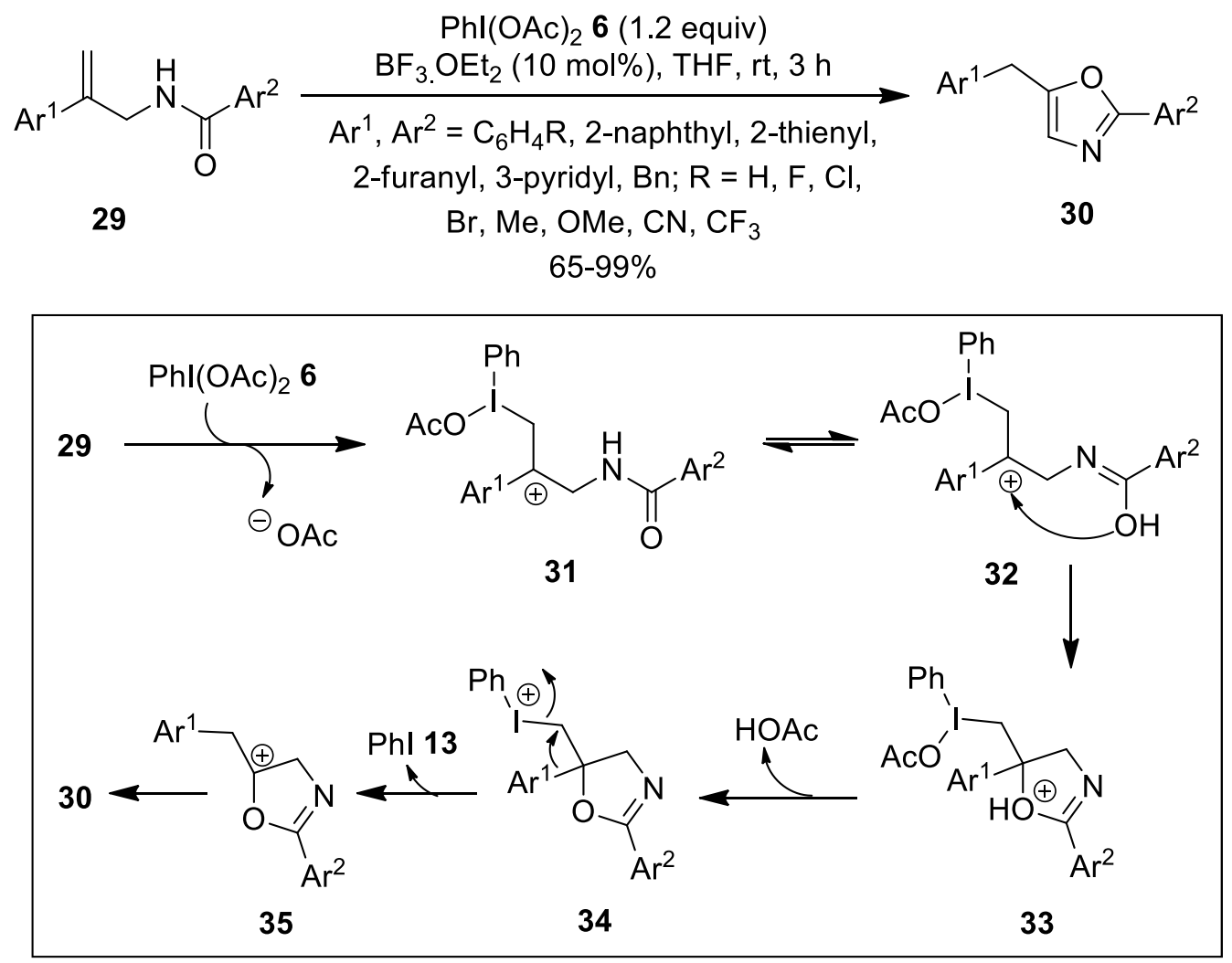

Scheme 7. PIDA-mediated oxidative rearrangements of amides 29 to oxazoles $\mathbf{3 0 .}$ 
Another interesting heterocycle, oxazoline 37, was synthesized by Ranjith et al. via PIDA-mediated intramolecular oxyacetoxylation of substituted $N$-allylamides 36 using HF.py as the promoter (Scheme 8). ${ }^{44}$ Reaction mechanism initiates with the conversion of PIDA 6 into aryliodinium ion 38 influenced by HF.py, which further interacts with the alkene to form cyclic iodonium ion 39. Next, exo attack by the amide moiety transforms cyclic iodonium ion 39 into alkyl iodane 40. Finally, nucleophilic attack by acetyl group liberates PhI 13 and delivers oxazolines $\mathbf{3 7}$ following $\mathrm{S}_{\mathrm{N}} 2$-like bimolecular reductive elimination.
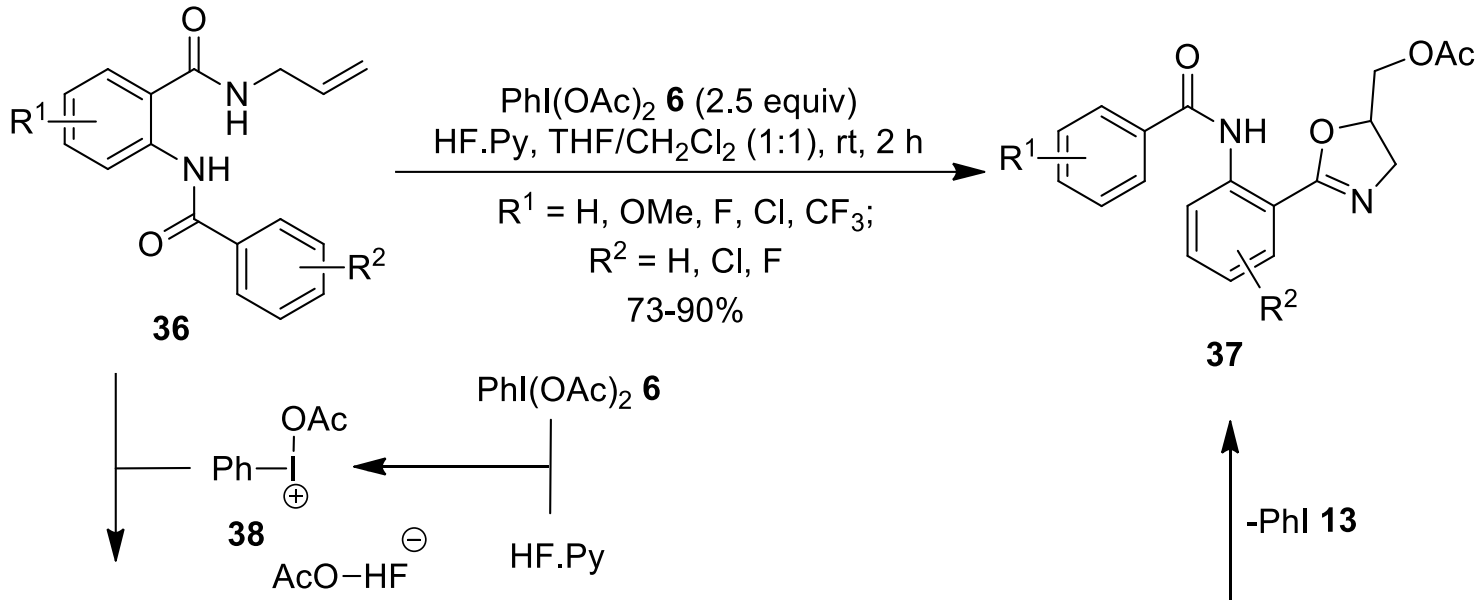<smiles>[R]c1cccc(NC(=O)c2ccccc2)c1C(=O)NCC1([R]([H])([H])[H])CCCCC1</smiles>

39

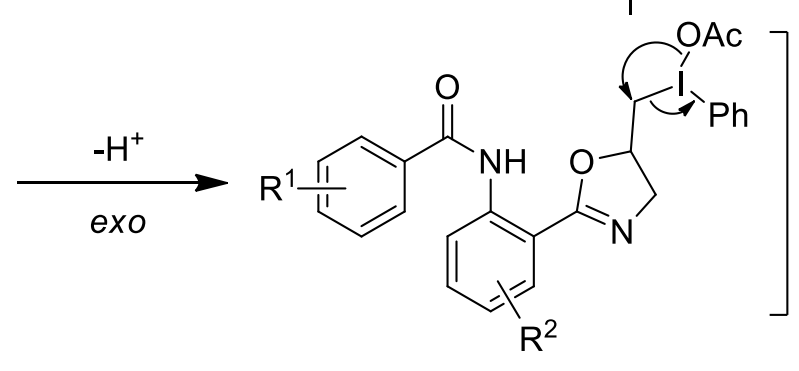

40

Scheme 8. Synthesis of substituted oxazolines 37 using PIDA 6 as an oxidant.

Later, two catalytic methods to prepare 2-oxazolines $\mathbf{4 1}$ and $\mathbf{4 3}$ were developed by Kamouka and Moran. ${ }^{45}$ The first method involves intramolecular cyclization of $N$-propargylamides 17 while other involves cyclization of $\beta$-amidoketones 42 using 2-iodoanisole 18 as precatayst with $m C P B A$ and $\mathrm{TsOH} .2 \mathrm{H}_{2} \mathrm{O}$ (Scheme 9). Both these approaches employ easily available starting materials, tolerates wide range of functional groups and operate under mild reaction conditions. The same group previously synthesized oxazolines through iodoarene-catalyzed cyclization of $\mathrm{N}$-alkenylamides using selectfluor as an oxidant. ${ }^{46}$ 
<smiles>[R]C#CCNC(=O)Br</smiles>

17

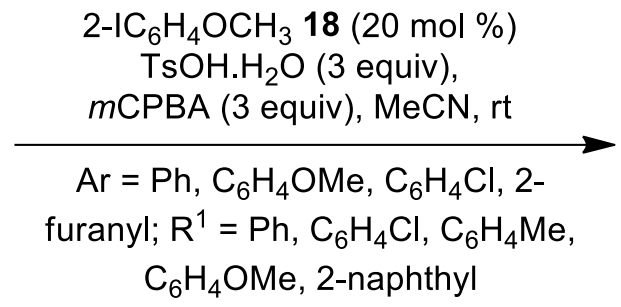

$35-82 \%$<smiles>[R]C(=O)C1CN=C(Br)O1</smiles>

41<smiles>[R]CC([R])C([R])CNC(=O)[Al]</smiles>

42
2- $-\mathrm{IC}_{6} \mathrm{H}_{4} \mathrm{OCH}_{3} 18(20 \mathrm{~mol} \%)$

TsOH. $\mathrm{H}_{2} \mathrm{O}$ (3 equiv), $m$ CPBA ( 3 equiv), $\mathrm{MeCN}$, rt

$\mathrm{Ar}=\mathrm{Ph}, \mathrm{C}_{6} \mathrm{H}_{4} \mathrm{OMe} ; \mathrm{R}^{1}=$ Aryl, $\mathrm{Me}, \mathrm{Et} ; \mathrm{R}^{2}=\mathrm{Me}, \mathrm{Ph}$

$46-95 \%$<smiles>[R]C(=O)C1CN=C([Al])O1</smiles>

43

Scheme 9. 2-lodoanisole-catalyzed cyclization of $\mathrm{N}$-propargylamides $\mathbf{1 7}$ and $\mathbf{B}$-amidoketones $\mathbf{4 2 .}$

In 2018, Liu and co-workers designed a simple and efficient method for the preparation of oxazolines 44 via 5-exo-dig process (Scheme 10). ${ }^{41}$ In the presence of PIDA 6 (1.0 equiv) and Lil (1.0 equiv), iodocyclization of various $N$-propargylamides 17 was performed, providing iodomethylene-2-oxazolines 44 in significant yields. Synthesis of 5-halomethyloxazolines was previously accomplished by the same group through PIDA-promoted cyclization of $\mathrm{N}$-allylamides. ${ }^{47}$

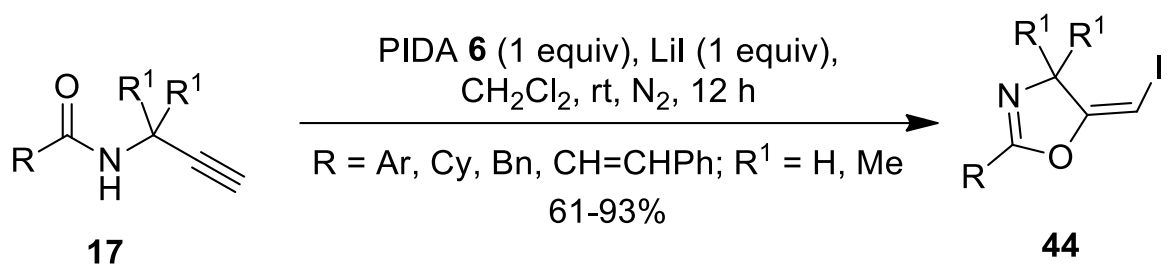

Scheme 10. PIDA-induced preparation of oxazolines 44.

Later, another iodine(III)-mediated route to prepare oxazolines $\mathbf{4 7}$ was developed by Hong and co-workers by treating $N$-allylamides $\mathbf{4 5}$ with bis(sulfonyl)imides $\mathbf{4 6}$ as the nitrogen source (Scheme 11). ${ }^{48}$ The proposed mechanism for this inter-/intra aminohydroxylation reaction involves in situ generation of PhI(OAC)(NR $\left.{ }^{1} R^{2}\right)$ or $\mathrm{PhI}\left(N R^{1} R^{2}\right)_{2} \mathbf{4 8}$ which activates double bond of $\mathbf{4 5}$ followed by subsequent cyclization and substitution to yield heterocyclic products $\mathbf{4 7}$. Several electron deficient amines $\mathbf{4 6}$ were evaluated and validated that the desired reactivity originates from attached (benzene)sulfonyl group. 


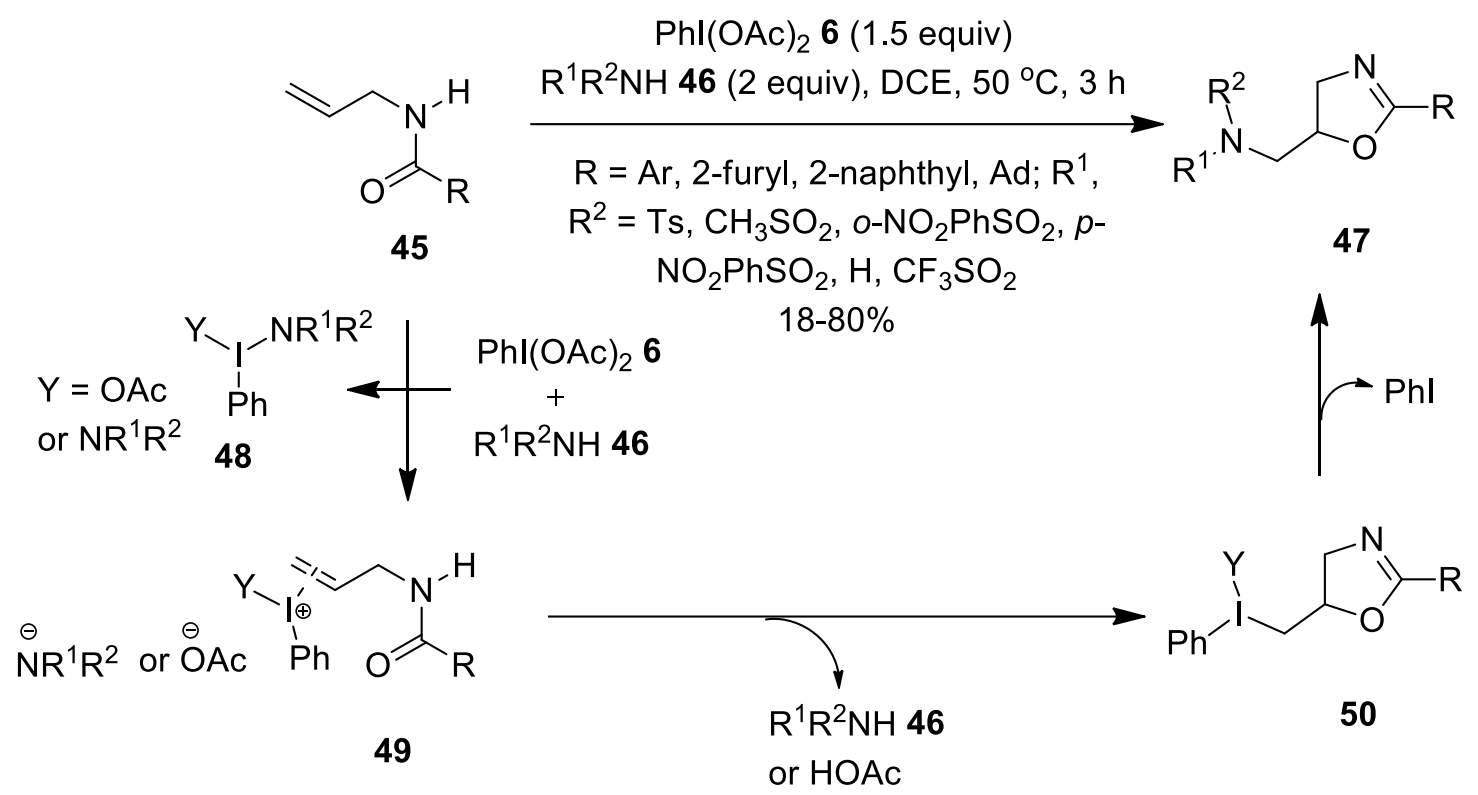

Scheme 11. Synthesis of oxazolines 47 using PIDA 6 as an oxidant.

Further, Scheidt et al. provided a direct route to 2-oxazolines $\mathbf{5 2}$ incorporating a fluoromethyl group from $\mathrm{N}$-allylcarboxamides $\mathbf{4 5}$ following I(I)/I(III) catalysis (Scheme 12). ${ }^{49}$ The success of this fluorooxygenation reaction lies in its efficient generation of active iodine(III) species, $p$-TolIF 219 in situ from precatalyst 4-iodotoluene $\mathbf{5 1}$ using Selectfluor as an oxidant. The amine/HF ratio of 1:4.5 was obtained by combining $\mathrm{Et}_{3} \mathrm{~N} \cdot 3 \mathrm{HF}$ and Olah's reagent (Pyr·HF).<smiles>[R]C(=O)NCC=C</smiles>

45

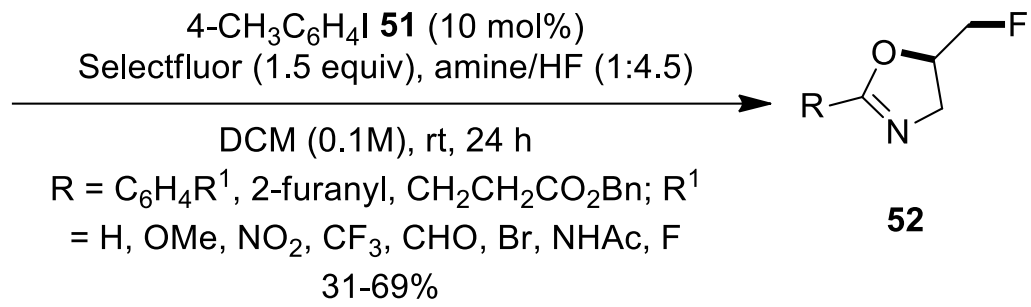

Scheme 12. 4-iodotoluene-catalyzed fluorocyclization of $\mathrm{N}$-allylcarboxamides $\mathbf{4 5}$ to form 2-oxazolines $\mathbf{5 2 .}$

In the same year, a convenient synthesis of 2-oxazolines 56 via PIDA-promoted cyclization of imine intermediate 55 was demonstrated by Carlucci et al. (Scheme 13). ${ }^{50}$ The imines $\mathbf{5 5}$ were obtained subsequently by treating amino alcohols $\mathbf{5 3}$ with aldehydes $\mathbf{5 4}$ in methanol solution. Also, synthesis of 3-oxazolines was accomplished albeit in lower yields under similar reaction conditions. 


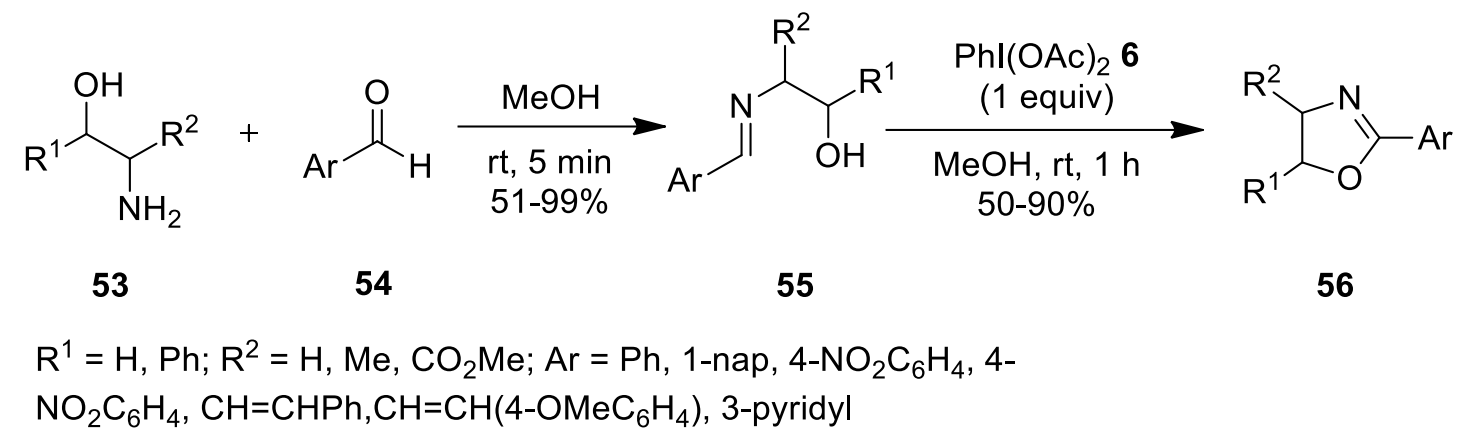

Scheme 13. PIDA-promoted synthesis of 2-oxazolines 56.

2.1.2.2. Synthesis of Isoxazole and Isoxazolines. In 2016, Peddinti and co-workers developed a strategy to access isoxazole derivatives $\mathbf{6 0}$ via PIDA-mediated [3+2] cycloaddition of in situ formed nitrile oxides $\mathbf{5 8}$ from aldoximes 57 with alkynes 59 (Scheme 14). ${ }^{51}$ Scope of the reaction was explored using dimethyl acetylenedicarboxylate (DMAD), diethyl acetylenedicarboxylate (DEAD) and methyl propiolate as dipolarophiles. Different substituents on aryl moiety of aldoximes $\mathbf{5 7}$ were well tolerated and anticipated products were isolated in shorter reaction time.

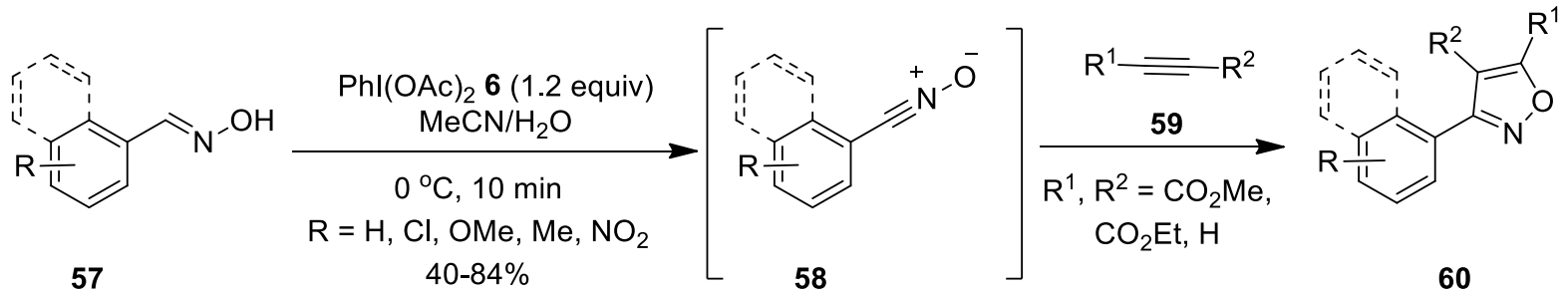

Scheme 14. Synthesis of isoxazole derivatives 60 using PIDA 6 as an oxidant.

Later in 2019, Kobayashi and Togo reported one-pot synthesis of 3-aryl- or 3-alkylisoxazoles 63 through the reaction of primary alcohols 61 with $\mathrm{PhI}(\mathrm{OAc})_{2} 6$ and then sequential reactions with $\mathrm{NH}_{2} \mathrm{OH}, \mathrm{NCS}$ and alkynes 59 (Scheme 15). ${ }^{52}$ This reaction involves PIDA-mediated oxidation of primary alcohols 61 to aldehydes which reacts with hydroxylamine $\mathbf{6 2}$ to form oximes $\mathbf{5 7}$ (Step 1 and 2). Further, reaction of oximes $\mathbf{5 7}$ with NCS generates nitrile $N$-oxides in situ which then reacts with alkynes 59 to yield isoxazoles 63 via 1,3-dipolar cycloaddition (Step 3 and 4). Additionally, synthesis of 3-aryl-/3-alkylpyrazoles was achieved by replacing $\mathrm{NH}_{2} \mathrm{OH} \cdot \mathrm{HCl}$ with $\mathrm{NH}_{2} \mathrm{NHPh}$ in the second step under similar conditions.

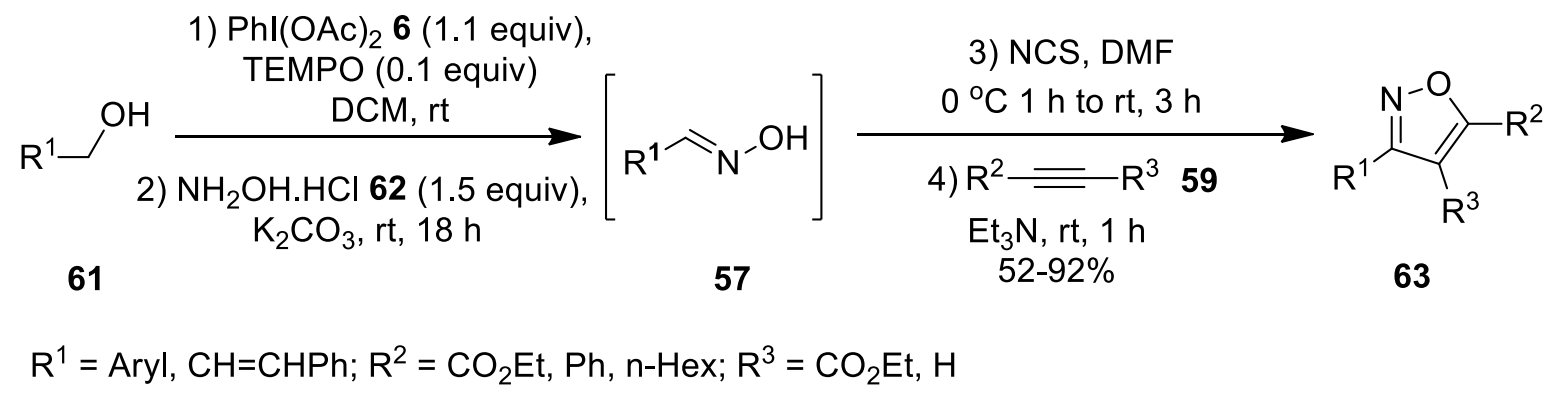

Scheme 15. PIDA-mediated synthesis of 3-aryl- and 3-alkylisoxazoles 63 from primary alcohols 61. 
Subsequently, Mukthar and co-workers described synthesis of flavone- and coumarin-based isoxazoles 65 and 67 through one-pot reaction of aryl aldehydes 54, hydroxylamine 62 and 3-O-propargylflavones 64/3-Opropargylcoumarin 66 via PIDA-mediated sequential oxidative cyclization and [3+2] cycloaddition reaction (Scheme 16). ${ }^{53}$ Further synthesis of tri-substituted isoxazoles 68 was accomplished by using dimethyl acetylenedicarboxylate (DMAD) $\mathbf{5 9}$ as an alkyne source. High product yields, excellent functional group tolerance, shorter reaction time, easy-workup and purification are key advantages of developed protocol. Further, synthesized compounds have been tested for the antibacterial activity.

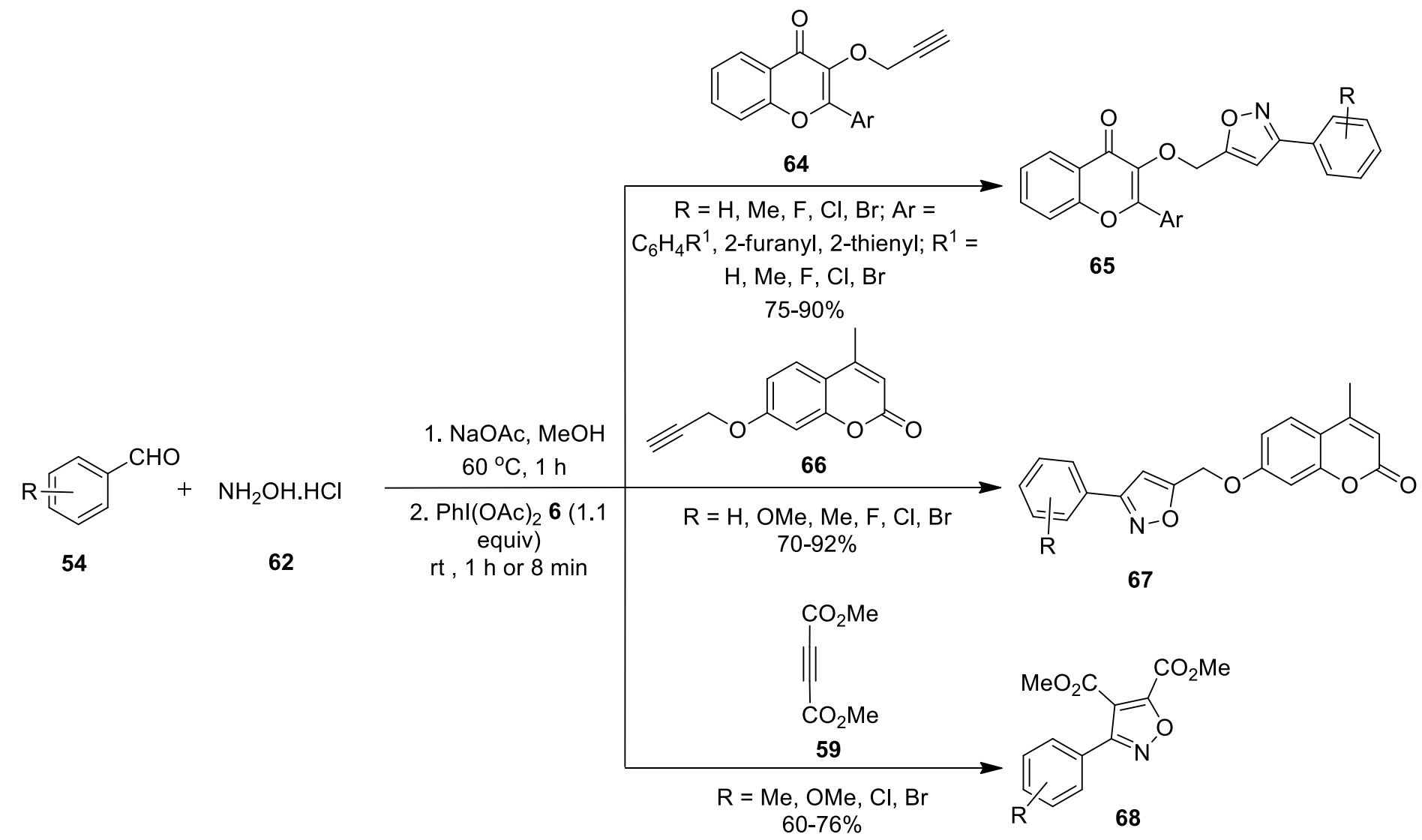

Scheme 16. PIDA-induced one-pot synthesis of isoxazoles 65,67 and 68.

Park et al. constructed isoxazolines $\mathbf{7 0}$ through PIDA-induced Ritter-type amidation of terminal olefins 69. Acetonitrile plays dual role of solvent and the amine source (Scheme 17). ${ }^{54}$ Notably, activation of PhI(OAc) 26 by $\mathrm{BF}_{3} \cdot \mathrm{OEt}_{2}$ generates active iodine(III) species in situ, that reacts with 69 to form electrophilic iodonium intermediate $\mathbf{7 1}$ which could give desired product $\mathbf{7 0}$ through sequential 5-exo-type cyclization and Ritter-typesubstitution using excess acetonitrile as the nucleophile. A variety of ketone oximes with aryl, heteroaryl and alkyl substituents were well tolerated. Specifically, electron-deficient aryl ketone oximes displayed robust reactivity thereby giving corresponding products in moderate yields while electron-rich ones gave inferior results. Further a similar method for the construction of heteroatom-containing isoxazolines 73 was demonstrated by Cai and co-workers. ${ }^{55}$ This cascade reaction featured PIDA-mediated sulfeno-/seleno/functionalization of several $\beta, \gamma$-unsaturated oximes 69 using substituted disulfides/diselenides 72 as $S / S e-$ sources. 


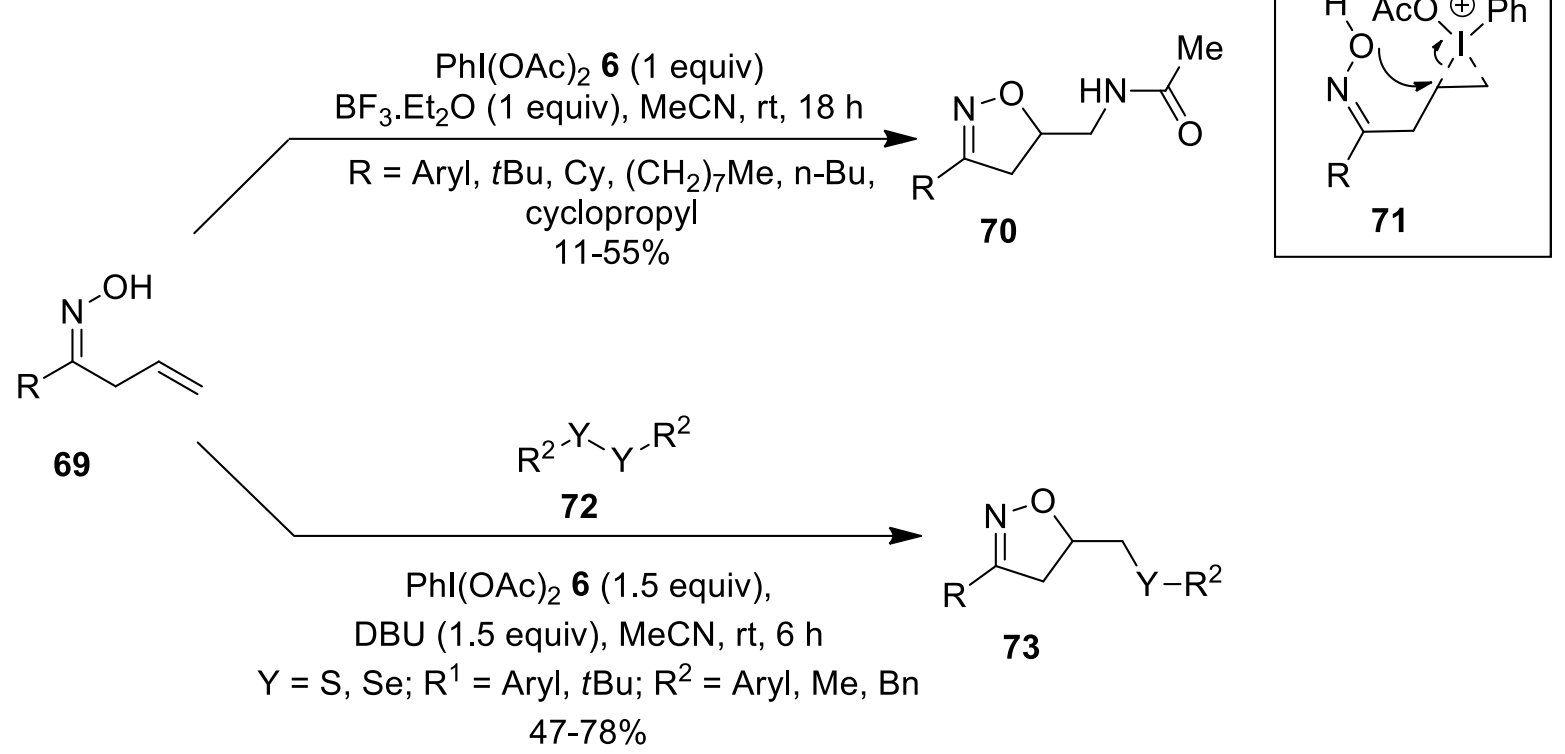

Scheme 17. Preparation of isoxazolines $\mathbf{7 0}$ and $\mathbf{7 3}$ from $\beta, \gamma$-unsaturated oximes 69 using PIDA 6.

2.1.2.3. Synthesis of oxadiazoles. Meanwhile, Zhdankin's research group reported convenient synthesis of 1,2,4-oxadiazoles 77 via oxidative cycloaddition of substituted aldoximes $\mathbf{5 7}$ with nitriles $\mathbf{7 4}$ using 2-iodosylbenzoic acid triflate (IBA-OTf) 75 as stoichiometric oxidant (Scheme 18). ${ }^{56}$ The reagent IBA-OTf 75 was previously prepared by the same group from iodosylbenzoic and trifluoromethanesulfonic acid. ${ }^{57}$ Further a catalytic system comprising 2-iodobenzoic acid 76 (5 mol \%) as precatalyst in the presence of $m$-CPBA and TfOH was also developed for the cyclization of aldoximes $\mathbf{5 7}$ and nitriles 74. Both stoichiometric and catalytic conditions gave desired products in moderate to high yields and electron-rich and -deficient substituents were well tolerated.

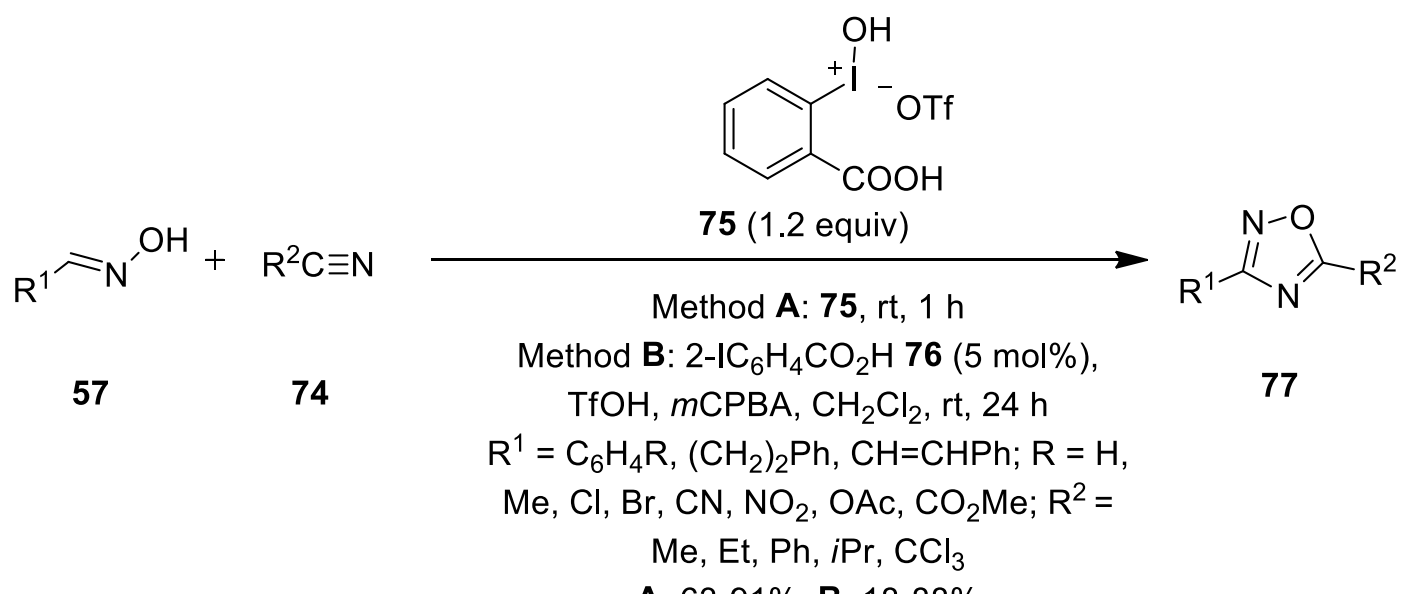

A: $63-91 \%$; B: $18-88 \%$

Scheme 18. Hypervalent iodine(III)-mediated oxidative cyclization of aldoximes 57 with nitriles $\mathbf{7 4}$ to yield 1,2,4oxadiazoles 77.

The mechanism for the catalytic reaction is depicted in Scheme 19. Cationic species $\mathbf{7 5}$ is formed in situ through oxidation of 2-iodobenzoic acid 76 by $m$-CPBA in the presence of TfOH. This active iodine(III) species 75 
reacts with aldoximes $\mathbf{5 7}$ via ligand exchange and generates nitrile oxides $\mathbf{7 9}$, which further react with nitriles $\mathbf{7 4}$ to deliver desired product $\mathbf{7 7}$. The regenerated precatalyst $\mathbf{7 6}$ is reoxidized by $m$-CPBA to continue the catalytic cycle.

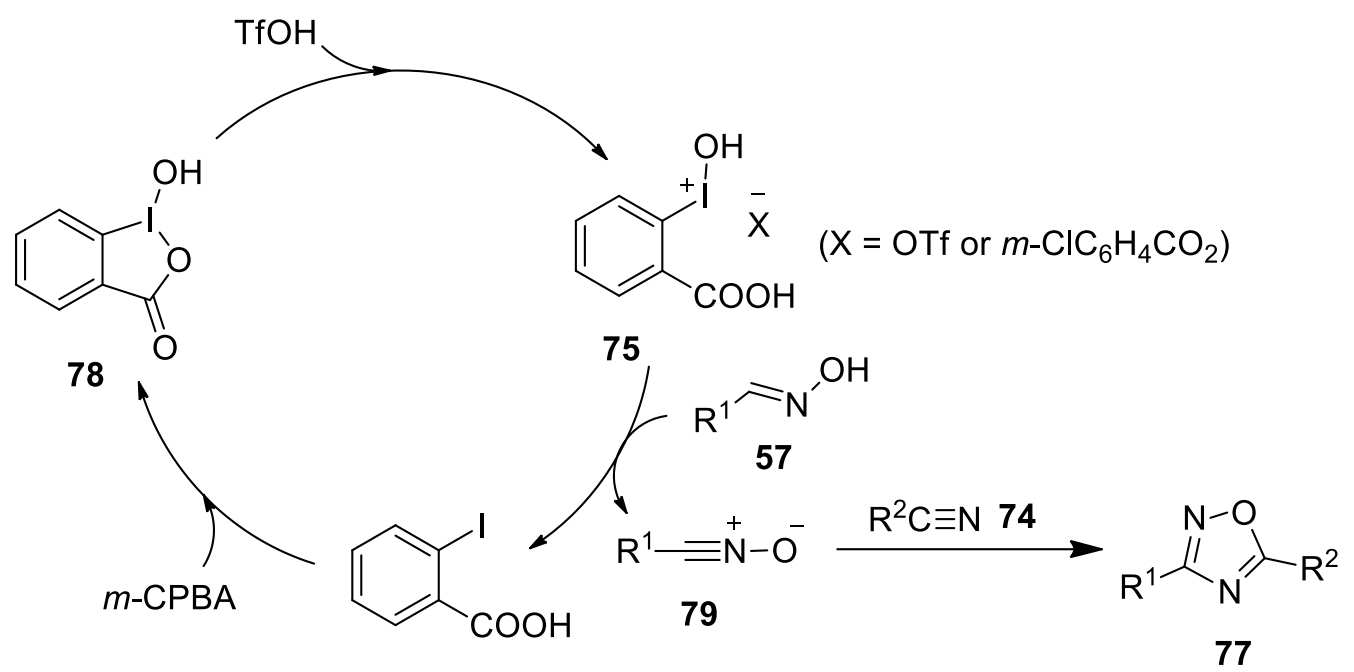

76

Scheme 19. The proposed catalytic cycle for the oxidative cyclization of aldoximes $\mathbf{5 7}$ using $\mathbf{7 6}$ as precatalyst.

In 2019, Sen and coworkers prepared 1,3,4-oxadiazoles 84 from variety of $N^{\prime}$-arylidene acetohydrazides $\mathbf{8 2}$ in the presence of isobutyraldehyde 83 and $p$-anisolyl iodide 18 . In this reaction, autoxidation of isobutyraldehyde $\mathbf{8 3}$ forms acyloxy radical that oxidizes $p$-anisolyl iodide $\mathbf{1 8}$ into active hypervalent iodine species in situ which promotes the cyclization reaction (Scheme 20). ${ }^{58}$ The precursors 82 were synthesized through condensation of variety of aromatic or heteroaromatic aldehydes $\mathbf{8 0}$ with acetyl, $p$-chlorobenzoyl, or tolyl hydrazides $\mathbf{8 1}$ in ethanol at room temperature. This method exhibits broad substrates scope and amenable for scale up reaction.

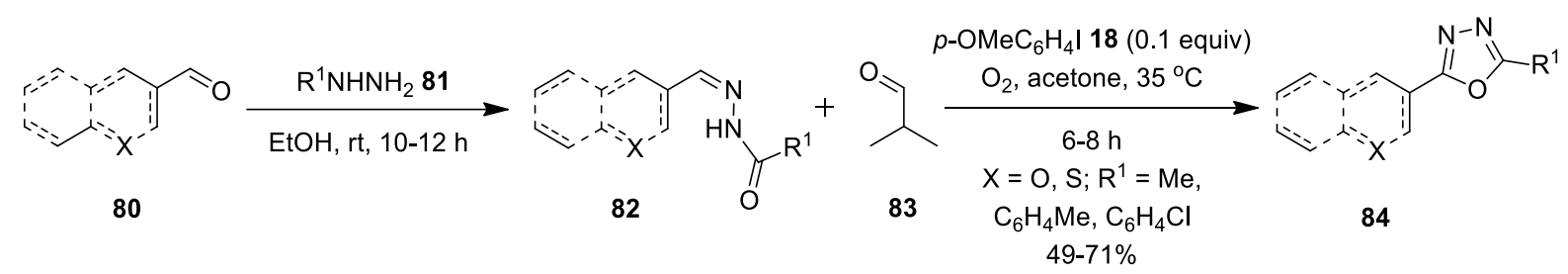

Scheme 20. Synthesis of 1,3,4-oxadiazole 84 using $p$-anisolyl iodide $\mathbf{1 8}$ as precatalyst.

The postulated mechanism for this transformation follows two main stages as shown in Scheme 21. In the preliminary stage, autoxidation of isobutyraldehyde $\mathbf{8 3}$ forms per acid $\mathbf{8 7}$ through acyl radical $\mathbf{8 5}$ and acyl peroxy radical $\mathbf{8 6}$ respectively. Further acyloxy radical $\mathbf{8 8}$ generated from acyl peroxy radical $\mathbf{8 6}$, enacts as oxidant for the oxidation of $p$-anisolyl iodide $\mathbf{1 8}$ leading to the in situ formation of hypervalent iodines(III) species $\mathbf{8 9 .}$. Finally, $\mathbf{8 9}$ react with substrate $\mathbf{8 2}$ to afford $\mathbf{9 0}$, which cyclizes to give $\mathbf{9 1}$ and aromatizes to afford $\mathbf{8 4}$. The regenerated $p$-anisolyl iodide $\mathbf{1 8}$ later continues the catalytic cycle. 


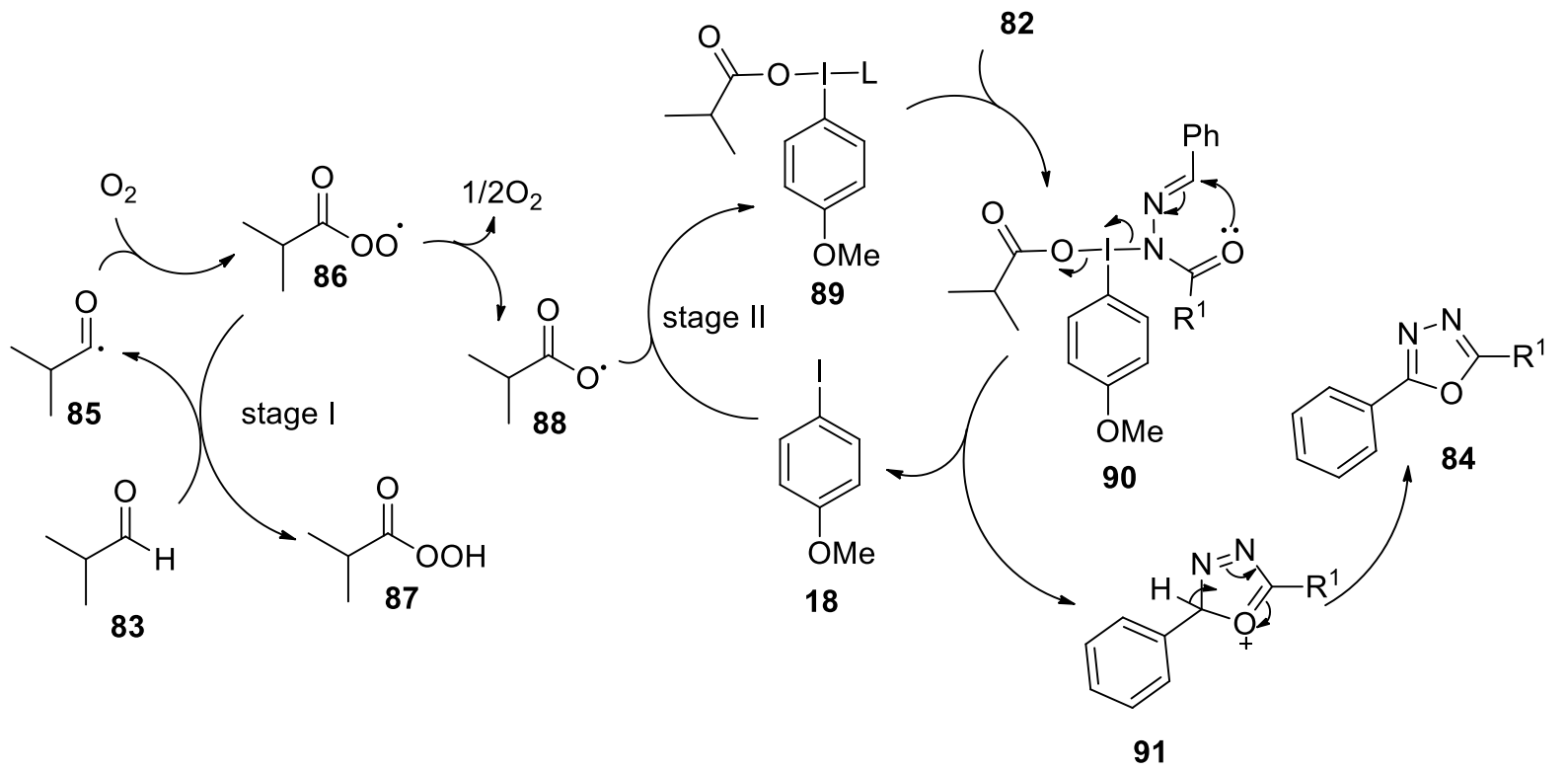

Scheme 21. The proposed catalytic cycle for the cyclization of $N^{\prime}$-arylidene acetohydrazides 82 using using $p$ anisolyl iodide 18 as the precatalyst.

2.1.2.4. Synthesis of Lactones. Liu and Shi have reported straightforward routes to access $\gamma$-lactones 93 via palladium (II)-catalyzed intramolecular dehydrogenative lactonization between carboxylic acids and $\mathrm{Y}-\mathrm{C}\left(\mathrm{sp}^{3}\right)-\mathrm{H}$ bond of substrates 92 using Phl(OAc) 6 as the oxidant and (pyridin-2-yl)isopropyl amine (PIP) as the directing group (Scheme 22). ${ }^{59}$ Among the various solvent and inorganic salts screened, toluene and Nal provided the best results. A variety of substituents on the alkyl chain of $\mathbf{9 2}$ were well tolerated. The proposed mechanism initiates with the $\mathrm{Pd}$-catalyzed activation of methylene $\mathrm{C}\left(\mathrm{sp}^{3}\right)-\mathrm{H}$ assisted by PIP-auxillary to form palladacycle 94, followed by PIDA-induced oxidation to give $\mathrm{Pd}(\mathrm{IV})$ intermediate 95 which undergoes ligand exchange to provide 96. Finally, $\mathbf{9 6}$ upon reductive elimination releases target product $\mathbf{9 3}$ and regenerates $\mathrm{Pd}(\mathrm{II})$ catalyst to complete the catalytic cycle. Also, lactone $\mathbf{9 3}$ could be generated via direct intramolecular $\mathrm{S}_{\mathrm{N}} 2$-type attack of carboxylate group onto the $\mathrm{Pd}(\mathrm{IV})-\mathrm{C}$ bond of intermediate 95 . 
<smiles>[R]CC(CC(=O)O)CC(=O)NC(C)(C)c1ccccn1</smiles>

92

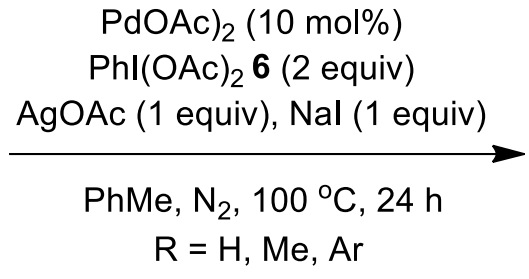

$32-77 \%$<smiles>[R]CC(=O)OC(C[R])CC(=O)NC(C)(C)c1ccccn1</smiles>

93

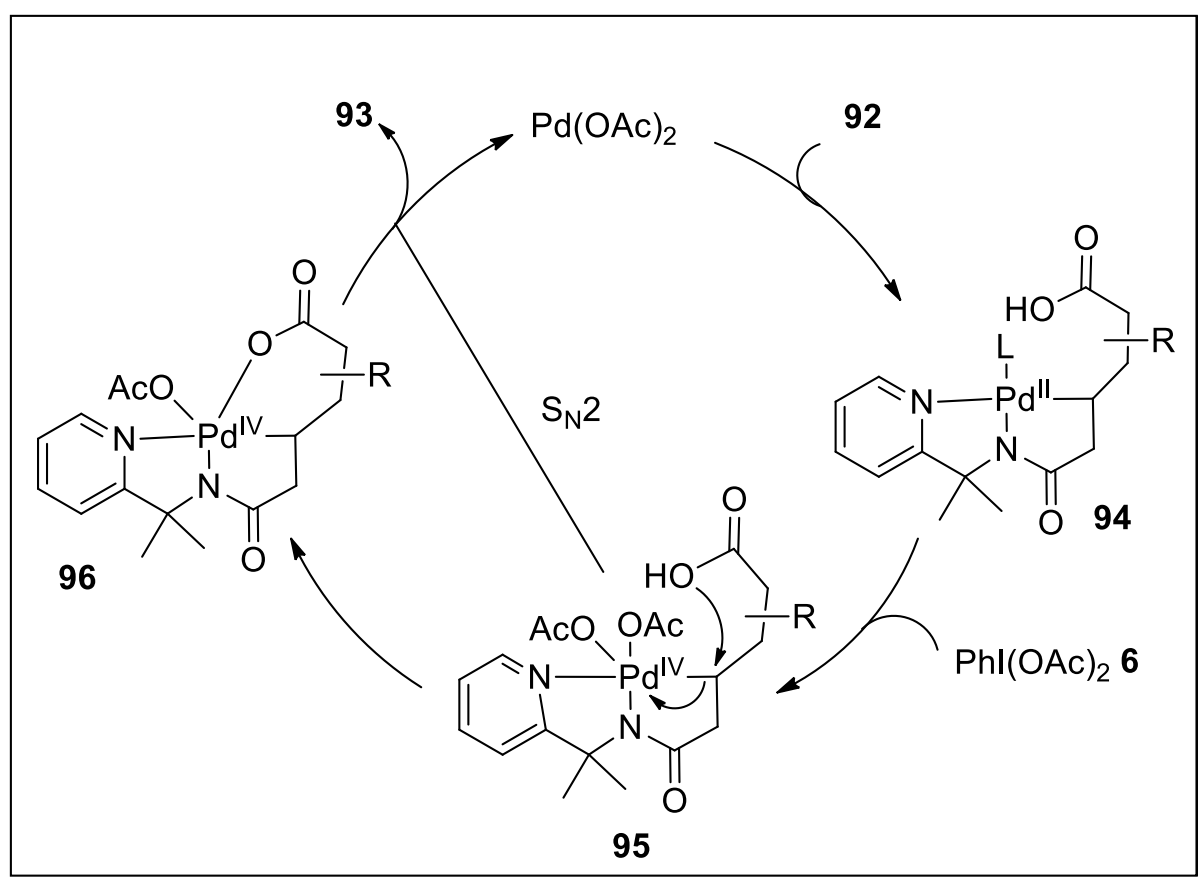

Scheme 22. Synthesis of $\gamma$-lactones 93 via intramolecular lactonization of 92 using $\mathrm{Phl}(\mathrm{OAc})_{2} 6$.

Further, Fujiwara and co-workers reported direct conversion of tetrahydrofuran-2-methanols 97 into $\gamma$ lactones 99 via oxidative cleavage by employing precatalyst, 2-iodobenzamide 98 in the presence of co-oxidant oxone (Scheme 23). ${ }^{60}$ Reaction proceeds through in situ generation of active hypervalent iodine(V) species 100 which facilitates the oxidation of substrates $\mathbf{9 7}$. This reaction occurs at room temperature under mild conditions without using any toxic heavy metals and desired products were isolated in significant yields. Later, the same group developed a new catalyst, [4-lodo-3-(isopropylcarbamoyl)phenoxy]acetic acid for the oxidation of tetrahydrofuran-2-methanols $\mathbf{9 7}$ and showed its reactivity greater than the previously employed catalyst $\mathbf{9 8}{ }^{61}$
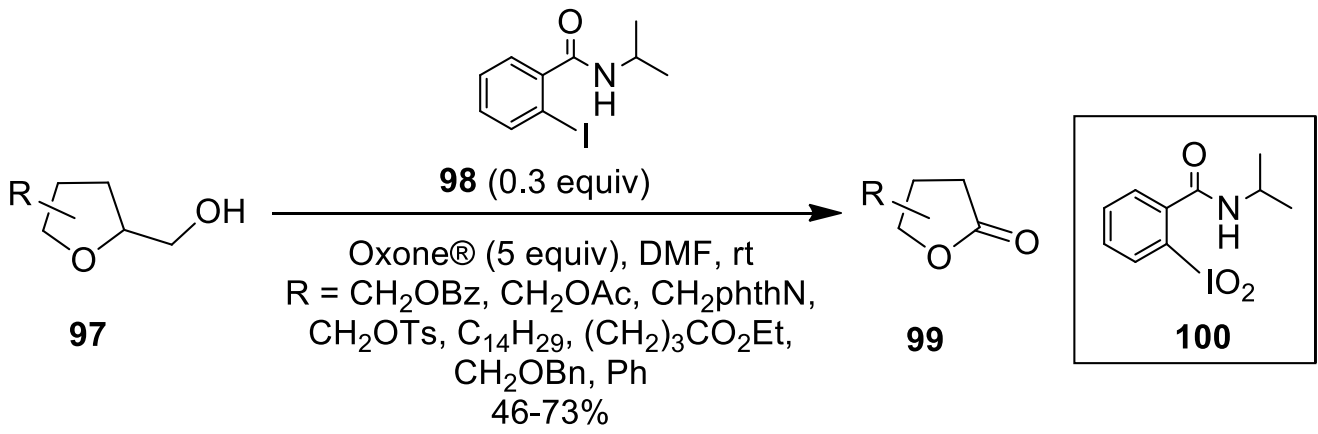

Scheme 23. Oxidation of tetrahydrofuran-2-methanols 97 to 99 using 100 as precatalyst. 
Another interesting strategy to prepare $y$-butyrolactones was reported by Gelis et al., featuring enantioselective $\gamma$-sulfonyl- and $\gamma$-phosphoryloxylactonatization of 4-pentenoic acid derivatives 101 (Scheme 24). ${ }^{62}$ Using stoichiometric or catalytic amount of $C_{2}$ symmetric chiral iodoarene 102, a facile synthesis of sulfonyloxy- $\gamma$-butyrolactones 104 or phosphoryloxy- $\psi$-butyrolactones 106 were achieved in variable yields. Notably, higher enantioselectivity was observed for phosphoryl-oxylactonatization (ee up to 93\%) as compared to the sulfonyloxylactonization of 4-pentenoic acids 101 (ee up to 84\%). Interestingly, gem-disubstituted-and spiro-lactones were obtained in high yields with good enantioselectivities.

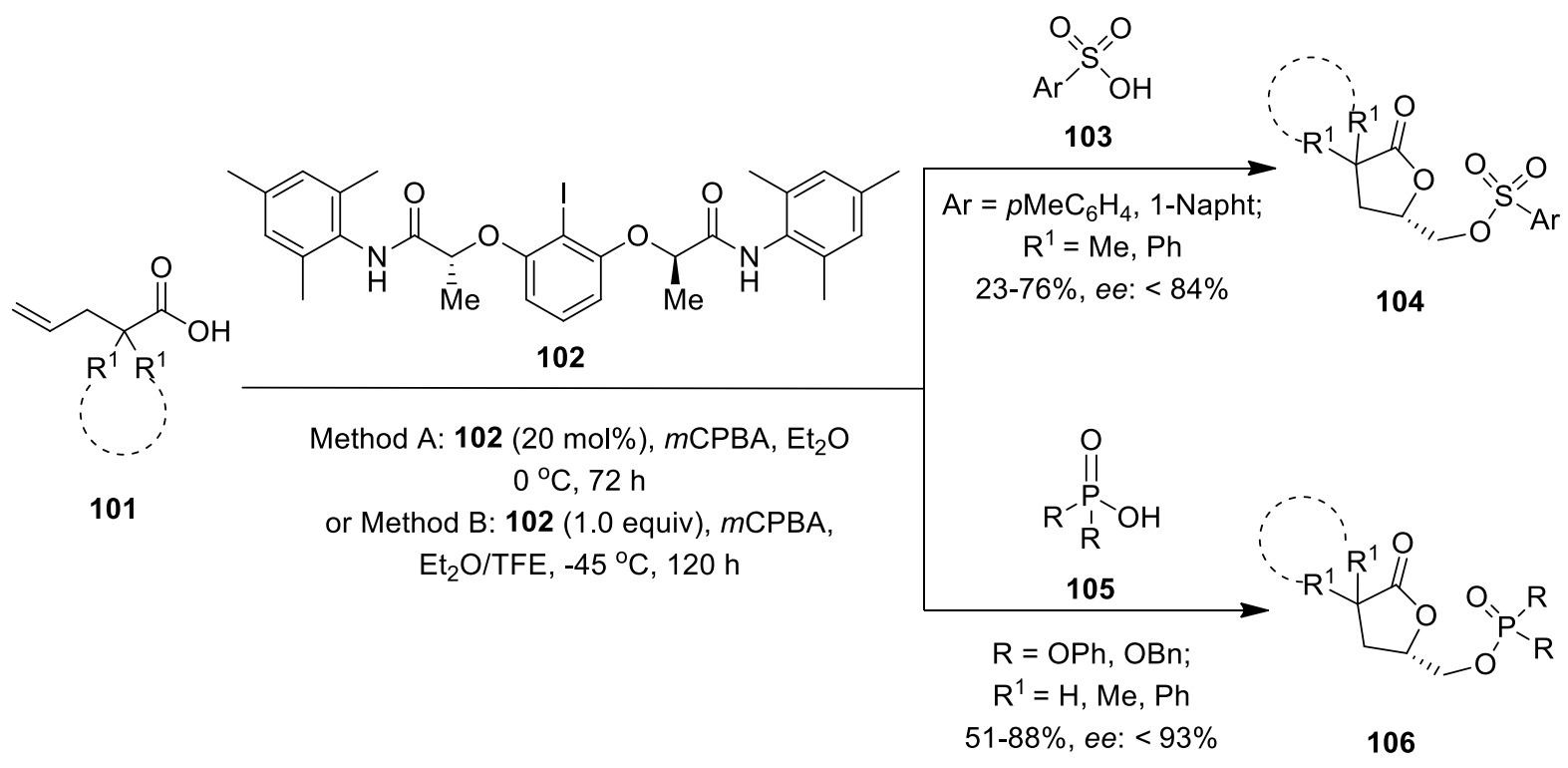

Scheme 24. Synthesis of $\gamma$-butyrolactones using chiral iodoarene 102 as precatalyst.

In 2017, Waser's research group synthesised (1,2)-azidolactones 109 through azidation and cyclization of unsaturated carboxylic acids 107 by employing azidobenziodoxolone (ABX) 108 as azide-transfer reagent with $0.5 \mathrm{~mol} \%$ of the $\mathrm{Cu}(\mathrm{dap}){ }_{2} \mathrm{Cl}$ as photoredox catalyst under blue LED irradiation (Scheme 25$) .{ }^{63}$ Both electron-rich and -deficient arenes as well as thiophene heterocycles were well tolerated. Furthermore, replacing ABX 108 with azidodimethylbenziodoxole (ADBX) $\mathbf{1 1 0}$ resulted in the formation of $(1,1)$-azidolactones 111 in the presence of Lewis acid catalyst, $\mathrm{Pd}(\mathrm{hfacac})_{2}(2 \mathrm{~mol} \%)$. Reactions were performed at room temperature, require low catalyst loading and exhibit broad substrate scope. 


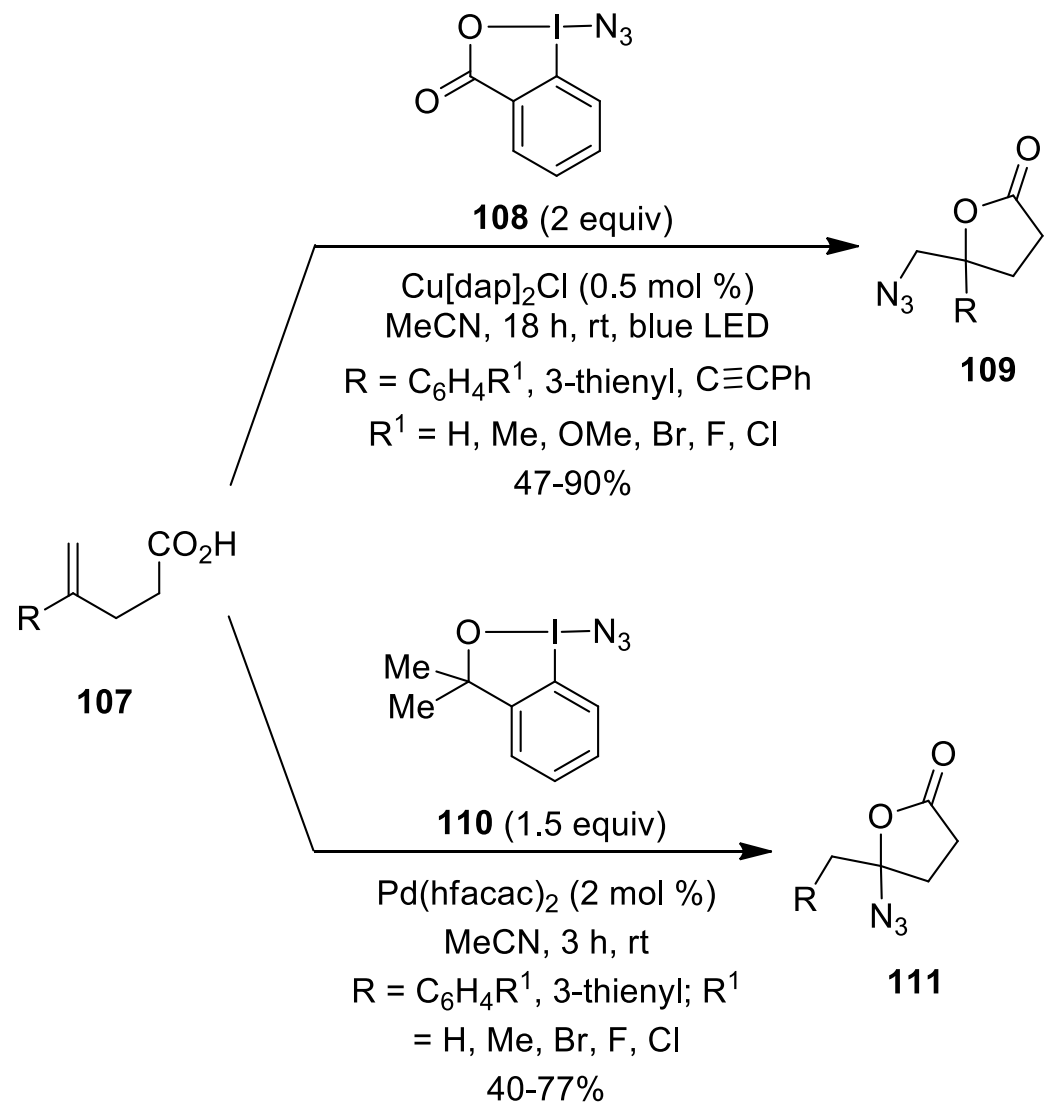

Scheme 25. Synthesis of azidolactones 109 and 111 using benziodoxol(on)e reagents 108 and 110 as azide source.

Minakata and co-workers investigated intramolecular lactonization of tertiary carbon containing carboxylic acids employing iodic acid $\left(\mathrm{HIO}_{3}\right) \mathbf{1 1 3}$ as an oxidant in the presence of catalyst $\mathrm{N}$-hydroxyphthalimide (NHPI) as hydrogen-atom transfer mediator (Scheme 26). ${ }^{64}$ Notably, NHPI is oxidized to the phthalimide N-oxyl radical which facilitates the site selective $\mathrm{C}-\mathrm{H}$ bond cleavage. The present oxidation system was found very effective for the preparation of $\gamma$-lactones $\mathbf{1 1 4}$ in respectable yields under metal-free conditions.

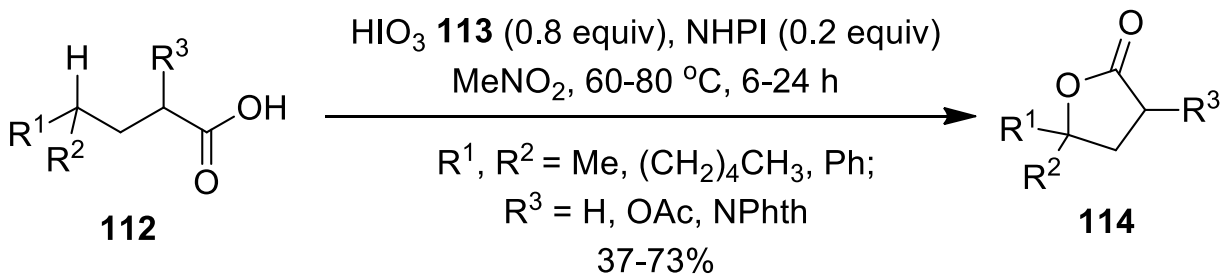

Scheme 26. Synthesis of $\gamma$-lactones 112 using iodic acid 114 as an oxidant.

The same team prepared furan-2(5H)-ones 116 via oxidative cyclization of various $\beta, \gamma$-unsaturated carboxylic acid derivatives 115 induced by hypervalent iodine reagent (Scheme 27). ${ }^{65}$ In this reaction, highly electrophilic species $\mathrm{Phl}(\mathrm{OTf})_{2}$ generated in situ from $\mathrm{Phl}(\mathrm{OAc})_{2} 6$ and $\mathrm{Me}_{3} \mathrm{SiOTf}$, plays crucial role of oxidant. Both aromatic and aliphatic substituents at the $\beta$-position were well tolerated. Further cyclization of 3-aryl-2,2- 
dimethylbut-3-enoic acids 117 in the presence of Phl(OTf) $)_{2}$ furnished furan-2(3H)-one products 118 in $54-68 \%$ yields.

$$
\begin{aligned}
& \mathrm{R}_{\mathrm{R}^{2}}^{\mathrm{CO}_{2} \mathrm{H}} \underset{\substack{\mathrm{R}^{1}=\mathrm{C}_{6} \mathrm{H}_{4} \mathrm{R}, \text { 1-naphthyl, 2-naphthyl, } \mathrm{Bn}, n-\mathrm{Bu} ; \mathrm{R}=\mathrm{H}, \mathrm{F}, \mathrm{Cl}, \mathrm{Br}, \mathrm{CF}_{3}, \mathrm{OMe}, \mathrm{Me} ;}}{\mathrm{Mhl}(\mathrm{OAc})_{2} 6 \text { (1 equiv) }} \\
& \mathrm{R}^{2}=\mathrm{H}, i \mathrm{Pr}
\end{aligned}
$$<smiles>[X]c1ccc(C(=C)C(C)(C)C(=O)O)cc1</smiles>

117

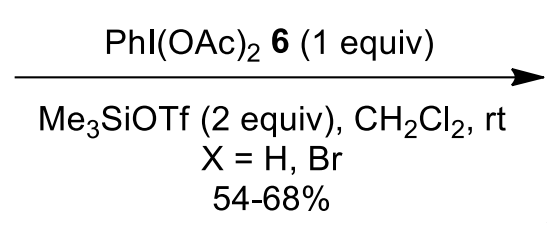

$54-68 \%$<smiles>[X]c1ccc(C2=COC(=O)C2(C)C)cc1</smiles>

118

Scheme 27. PIDA-induced synthesis of furan-2(5H)-ones 116 and furan-2(3H)-one 118.

2.1.2.5. Synthesis of pyrrolidines, dihydropyrroles and pyrroles. Wirth's research group disclosed thioamination of terminal alkenes 119 with [bis(trifluoroacetoxy)iodo]benzene $\left(\mathrm{Phl}\left(\mathrm{OCOCF}_{3}\right)_{2}\right) \mathbf{1 2 0}$ using thiolates 121 as an external nucleophile. This protocol provides flexible synthesis of pyrrolidines 123 in significant yields (Scheme 28). ${ }^{66}$ Further a stereoselective version of this reaction was developed by employing lactate-based chiral iodine(III) reagent 122, and thioamination products 123 were isolated in useful yields with up to $61 \%$ enantiomeric excess. Additionally, synthesis of indolines from corresponding $\mathrm{N}$-(2-allylphenyl)-4-methyl benzene sulfonamides was achieved under both conditions.

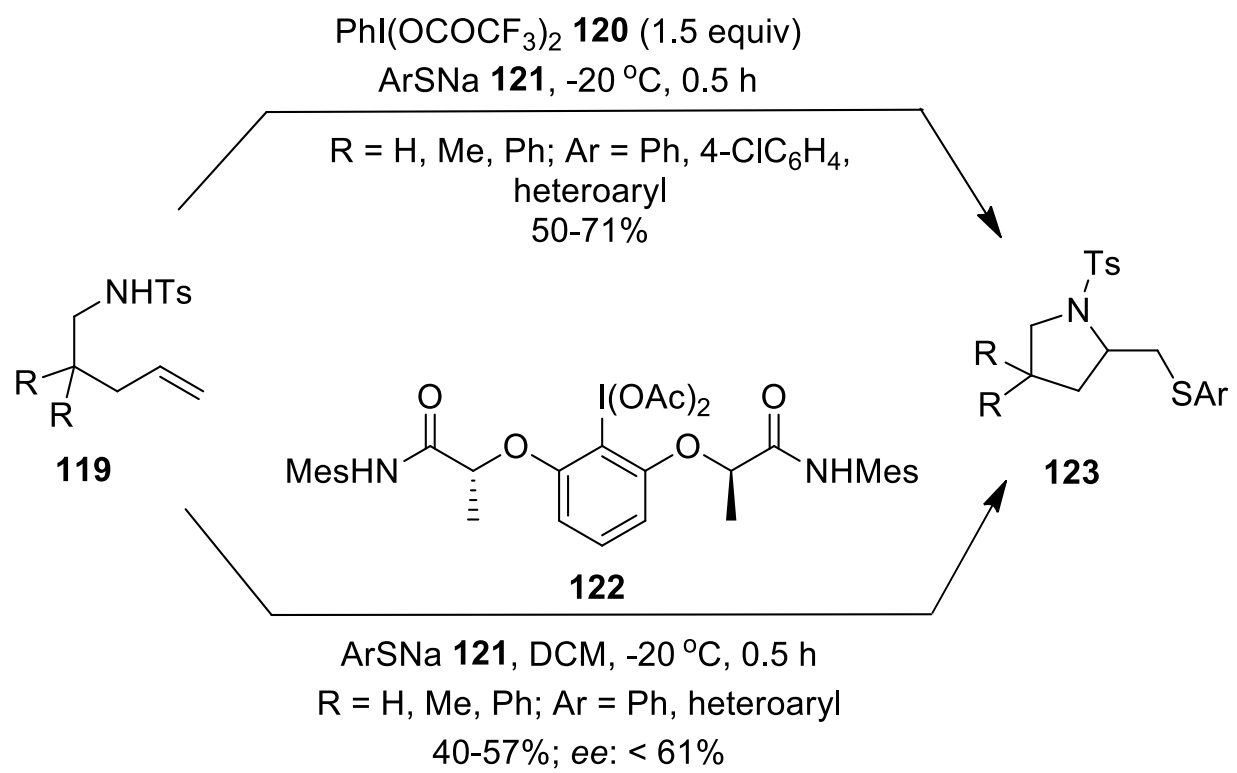

Scheme 28. lodine(III)-mediated synthesis of pyrrolidines 123. 
Later, Kitamura et al. synthesized $\mathbf{N}$-tosyl-3-fluoropyrrolidines $\mathbf{1 2 5}$ through intramolecular aminofluorination reaction of homoallylamines 124 using stoichiometric $\mathrm{PhI}(\mathrm{OAc})_{2} \mathbf{6}$ and pyridine.HF complex as a fluorine source (Scheme 29). ${ }^{67}$ Further catalytic aminofluorination was achieved using $p$-iodotoluene $\mathbf{5 1}$ as the catalyst in the presence of $\mathrm{Py} \cdot \mathrm{HF}$ and $m \mathrm{mCPBA}$. Moreover, synthesis of $\mathrm{N}$-tosyl-3-fluoropiperidine from $\mathrm{N}$-tosyl-4-pentenylamine was accomplished in $89 \%$ yield under identical conditions.

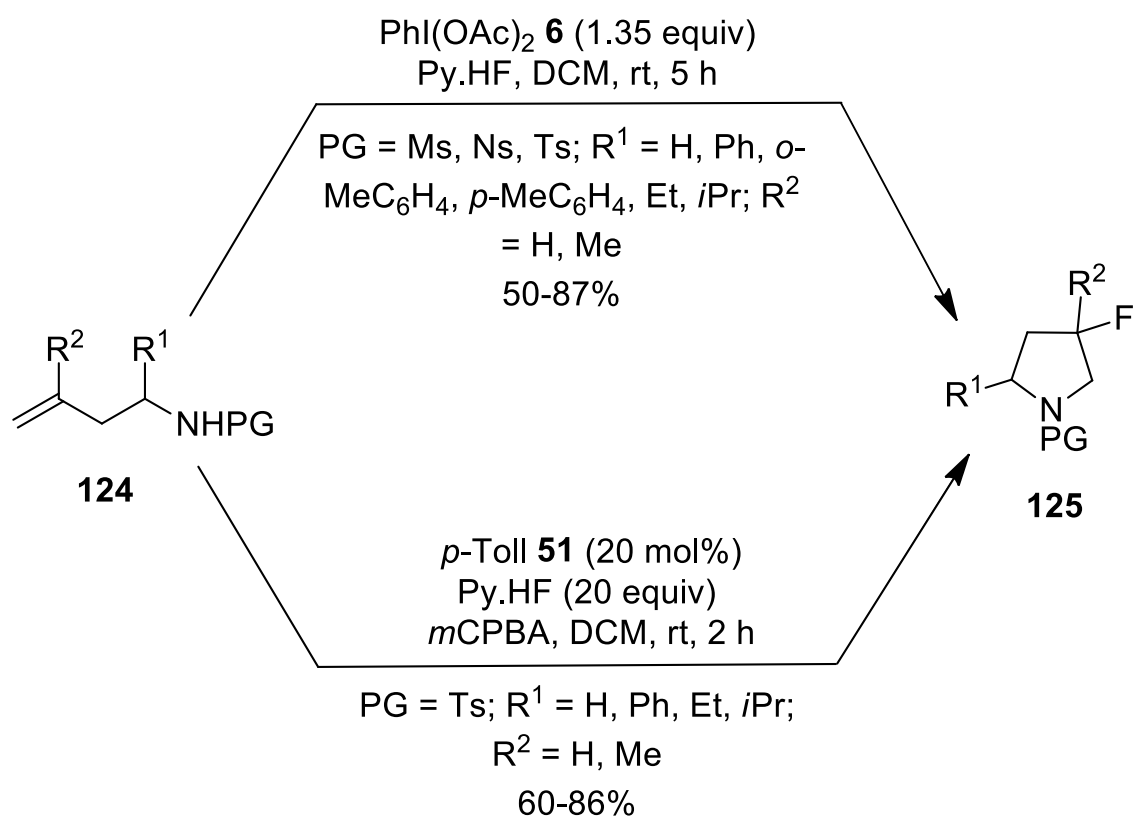

Scheme 29. Synthesis of $N$-tosyl-3-fluoropyrrolidines $\mathbf{1 2 5}$ from homoallylamines $\mathbf{1 2 4}$ using hypervalent iodine reagent.

Chang and co-workers prepared 2-aryl-1-pyrrolines 127 by treating 1-arylcyclobutanecarboxamides 126 with $\left(\mathrm{Phl}\left(\mathrm{OCOCF}_{3}\right)_{2}\right) 120$ via Hofmann rearrangement-ring expansion cascade reaction (Scheme 30). ${ }^{68}$ Substrates 126 with aromatic ring bearing ortho-, meta- and para substituents reacted cleanly under mild conditions to deliver products in 35-95\% yields. However, substrates with electron withdrawing groups delivered products in lower yields. Further this method has been adapted for the synthesis of 2,3-dihydro- $1 \mathrm{H}$ pyrrolo[2,1-a]isoquinolinium salts through cyclization of synthesized bromophenylpyrroline with alkynes using nickel catalyst.

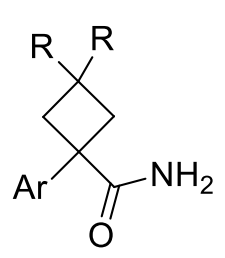

126

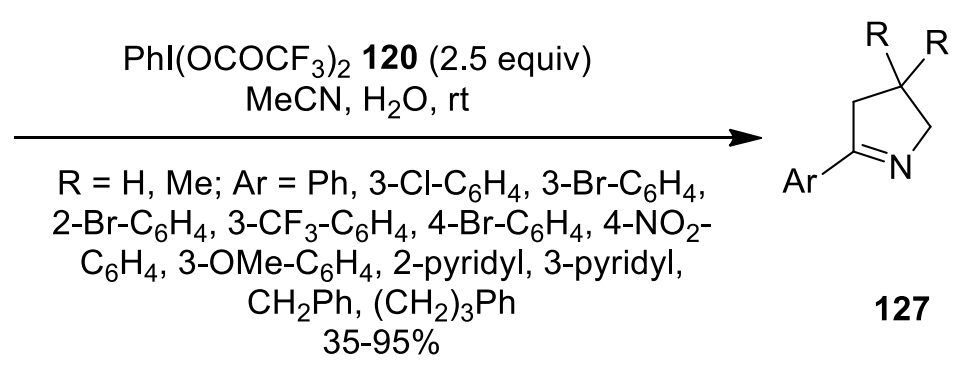

Scheme 30. Synthesis of 2-aryl-1-pyrroline derivatives 127 using Phl(OCOCF$)_{2} 120$ as an oxidant. 
Wang and co-workers demonstrated $\mathrm{I}_{2}$ /PIDA-promoted one-pot synthesis of polysubstituted trans-2,3dihydropyrroles $\mathbf{1 3 0}$ through multi-component reaction of aryl/alkyl amines $\mathbf{1 2 8}$ with alkyne esters $\mathbf{5 9}$ and chalcone derivatives 129 under ball-milling conditions (Scheme 31). ${ }^{69}$ Further using DDQ as an oxidant, one potthree step synthesis of multi-substituted pyrroles 131 were achieved under similar conditions. The present reaction featured broad substrates scope, shorter reaction time, and provides feasibility for larger-scale preparation.
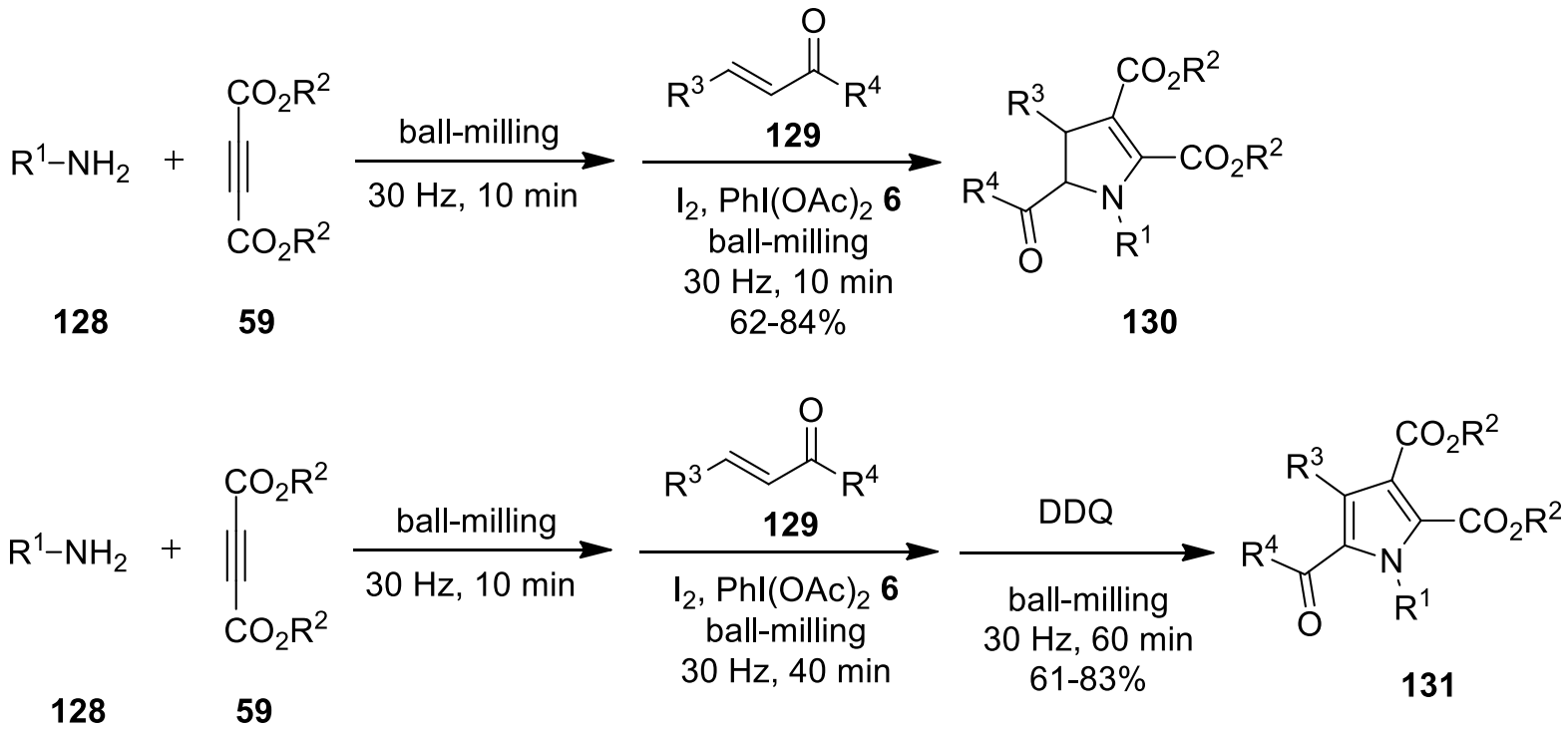

$\mathrm{R}^{1}=$ Aryl, $n$ Butyl, $n$ pent, $n \mathrm{Hex}, \mathrm{CH}_{2} i \mathrm{Pr}, \mathrm{Bn} ; \mathrm{R}^{2}=\mathrm{Et}, \mathrm{Me} ; \mathrm{R}^{3}, \mathrm{R}^{4}=$

$\mathrm{Ph}, \mathrm{C}_{6} \mathrm{H}_{4} \mathrm{Me}, \mathrm{C}_{6} \mathrm{H}_{4} \mathrm{OMe}, \mathrm{C}_{6} \mathrm{H}_{4} \mathrm{Cl}, \mathrm{C}_{6} \mathrm{H}_{4} \mathrm{Br}$, Me, 2-naphthyl

Scheme 31. Synthesis of dihydropyrroles 130 and pyrroles 131 using PIDA 6 as an oxidant.

The proposed reaction mechanism is shown in scheme 32. Initially, amine $\mathbf{1 2 8}$ reacts with alkyne ester $\mathbf{5 9}$ to give 8 -enamino ester 132, followed by Michael addition between 132 and 129 to give intermediate 133. Next, intermediate 133 reacts with $\mathrm{I}_{2}$ or in situ generated AcOI 134 from I $\mathrm{I}_{2}$ and PIDA 6 to yield iodide 135, which upon subsequent intramolecular $S_{N} 2$-type nucleophilic substitution affords polysubstituted trans-2,3-dihydropyrrole 130 with the elimination of HI. Finally, DDQ mediated dehydrogenation aromatization of 130 gives corresponding pyrrole 131. 


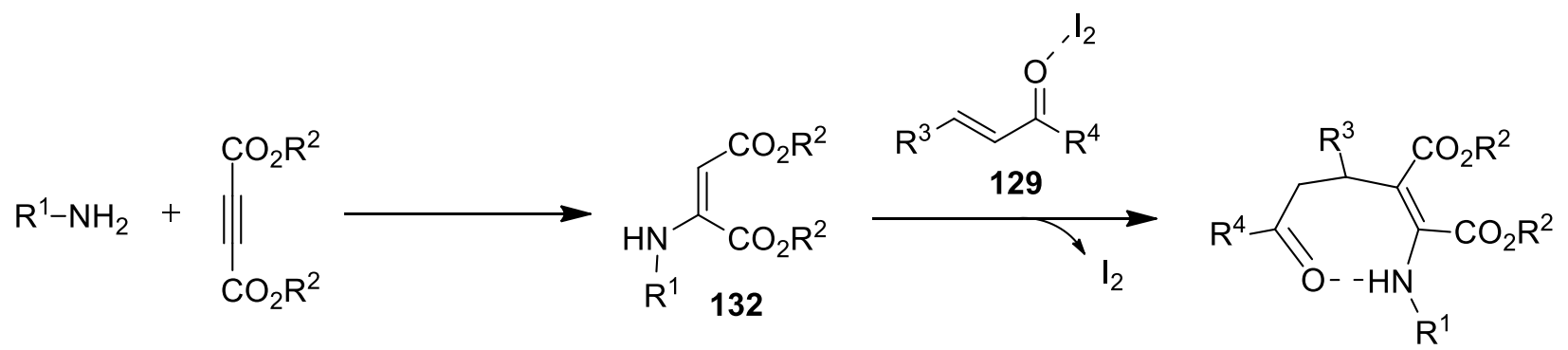

128

$\mathrm{I}_{2}+\mathrm{Phl}(\mathrm{OAc})_{2} 6$

DDQ

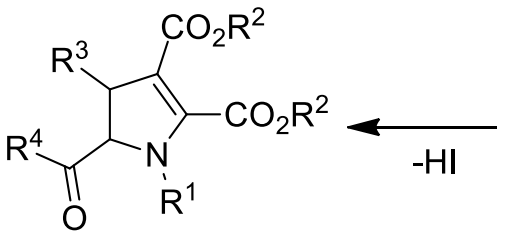

130
133

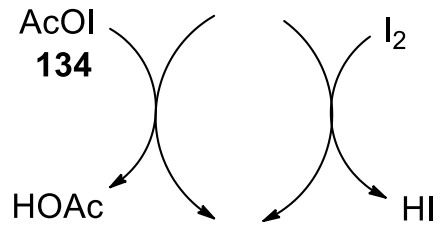

131

Scheme 32. The proposed mechanism for the synthesis of dihydropyrroles 130 and pyrroles 131 using PIDA 6 as an oxidant.

2.1.2.6. Synthesis of pyrazolines, imidazolines and imidazoles. In 2018, Park et al. explored Ritter-type amidoamidation of allyl ketone tosylhydrazones 136 by employing PIDA 6 as an oxidant and $\mathrm{BF}_{3} . \mathrm{OEt}_{2}$ as the promoter (Scheme 33). ${ }^{54}$ This reagent system in combination with acetonitrile as the solvent and the amine source has led to the synthesis of pyrazoline scaffolds $\mathbf{1 3 7}$ at ambient temperature. Proposed mechanism involved activation of PIDA 6 by the Lewis acid generating active hypervalent iodine(III) species in situ that forms cyclic iodonium intermediate $\mathbf{1 3 8}$ with the alkene, which subsequently undergoes 5-exo-type cyclization and Rittertype-substitution to deliver product 137.
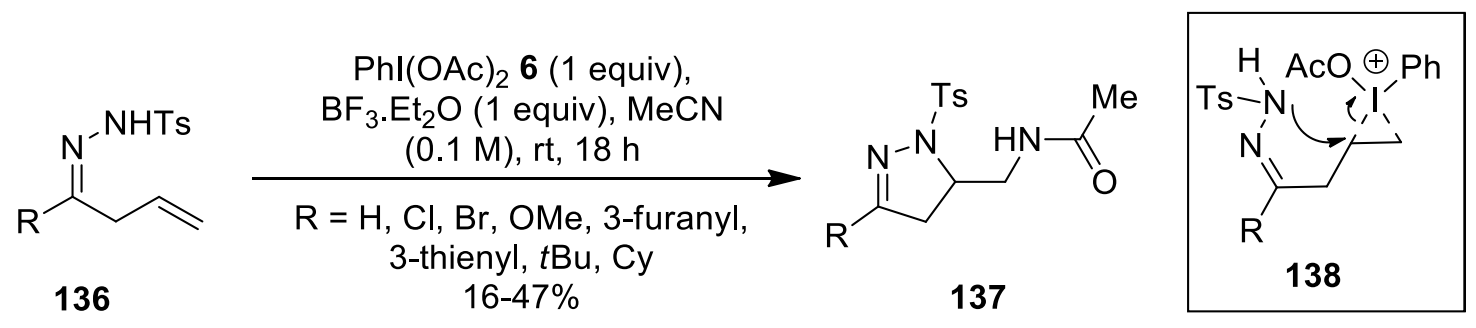

Scheme 33. Preparation of pyrazolines 137 using PIDA 6 as an oxidant.

Later, a similar methodology was developed for the construction of heteroatom-containing pyrazolines 139 from $\beta, \gamma$-unsaturated tosyl hydrazones 136 using PIDA 6 as the sole oxidant (Scheme 34). ${ }^{55}$ This cascade reaction proceeds through the generation of $\mathrm{N}$-centered radical $\mathbf{1 4 0}$ from corresponding $\mathrm{N}-\mathrm{H}$ bond, that undergoes sequential radical cyclization and sulfenylation/selenylation using disulfides/diselenides $\mathbf{7 2}$ as the S/Se- 
nucleophiles to form desired product 139. Aliphatic, aromatic and heteroaromatic disulfides/diselenides were well tolerated.

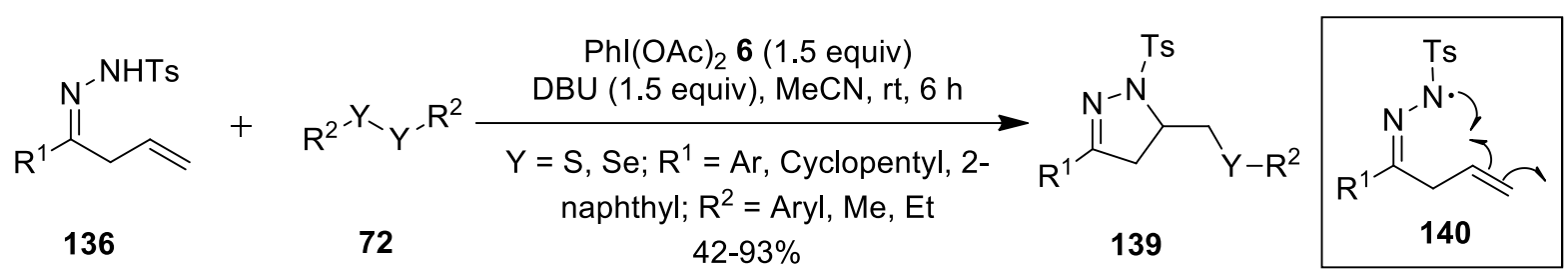

Scheme 34. PIDA-mediated synthesis of pyrazolines 139 using PIDA 6 as the sole oxidant.

In 2016, Chiba and co-workers reported iodine(III)-mediated intramolecular aminofluorination of $\mathrm{N}$-allylamidines 141 using $\mathrm{Et}_{3} \mathrm{~N} .3 \mathrm{HF}$ as the fluoride nucleophile (Scheme 35). ${ }^{70}$ This reaction enabled anti-selective preparation of 4-fluoroalkyl-2-imidazolines 143 from a series of di-, tri- and tetra-substituted $E$ - or Z-alkenes 141. Moreover, synthesized 2 -imidazoline moiety were subjected to reductive ring-opening to deliver 3fluoropropane-1,2-diamines in significant yields.

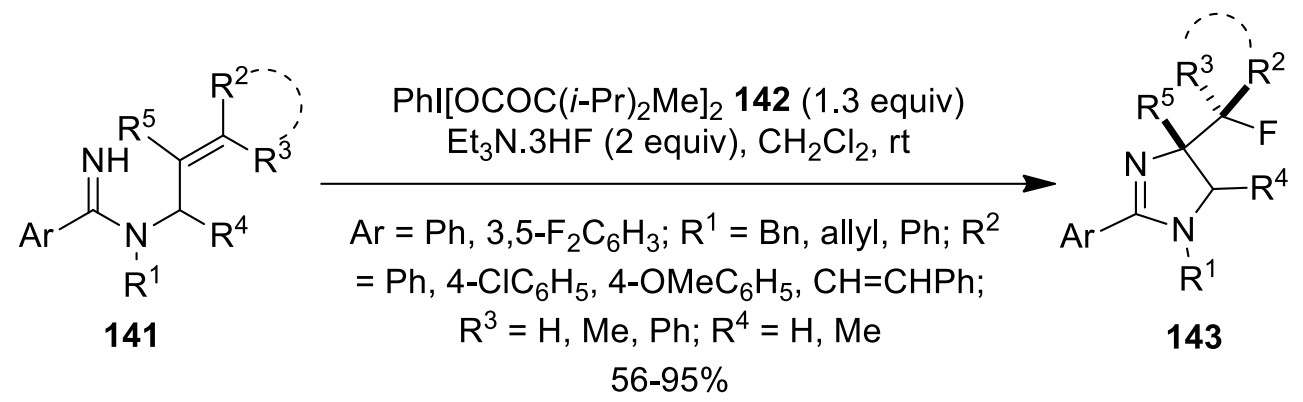

Scheme 35. Synthesis of 4-fluoroalkyl-2-imidazolines 143 using $\mathrm{Phl}\left[\mathrm{OCOC}(i-\mathrm{Pr})_{2} \mathrm{Me}\right]_{2} 142$ as an oxidant.

In the following year Yu's research group designed a domino azidation/C(sp $\left.{ }^{3}\right)-\mathrm{H}$ amination strategy for the transformation of $\mathrm{N}$-alkyl enamines 144 into 2,4,5-trisubsituted imidazoles 146 by reacting with TMSN 145 as an azide source in the presence of PIDA 6 under copper catalysis (Scheme 36). ${ }^{71}$ Presence of tetrabutyl ammonium iodide (TBAI) was essential for obtaining higher yields.

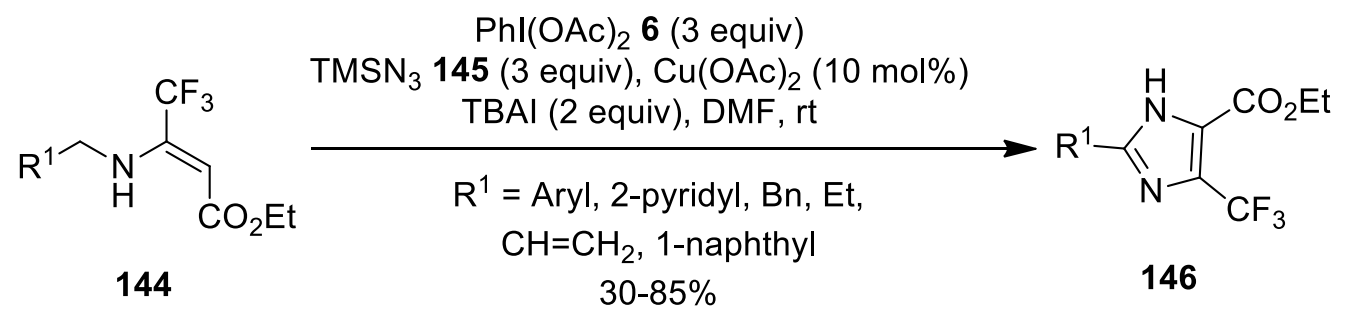

Scheme 36. Synthesis of 2,4,5-trisubsituted imidazoles 146 using PIDA 6 as oxidant.

2.1.2.7. Synthesis of lactam and imidazolidinones. Shen and Wang reported the first example of the introduction of a $\mathrm{CF}_{3}$-group onto the lactam ring 149 via $\mathrm{Cu}$-catalyzed intramolecular aminotrifluoromethylation 
of unsaturated amides 147 (Scheme 37). ${ }^{72}$ A series of $N$-methoxyamides 147 smoothly underwent 5-exo cyclization followed by $\mathrm{C}-\mathrm{N}$ bond formation by using Togni's reagent 148 to provide desired $\mathrm{CF}_{3}$-containing $\gamma^{-}$ lactam 149 in 48-82\% yields. Later, the same team demonstrated aminoazidation of several unactivated alkenes 147 by employing azidoiodinane 108 as an azide precursor. ${ }^{73}$ This diamination reaction enabled the installation of two distinct amino groups onto the alkenes with excellent regio- and stereoselectivity.

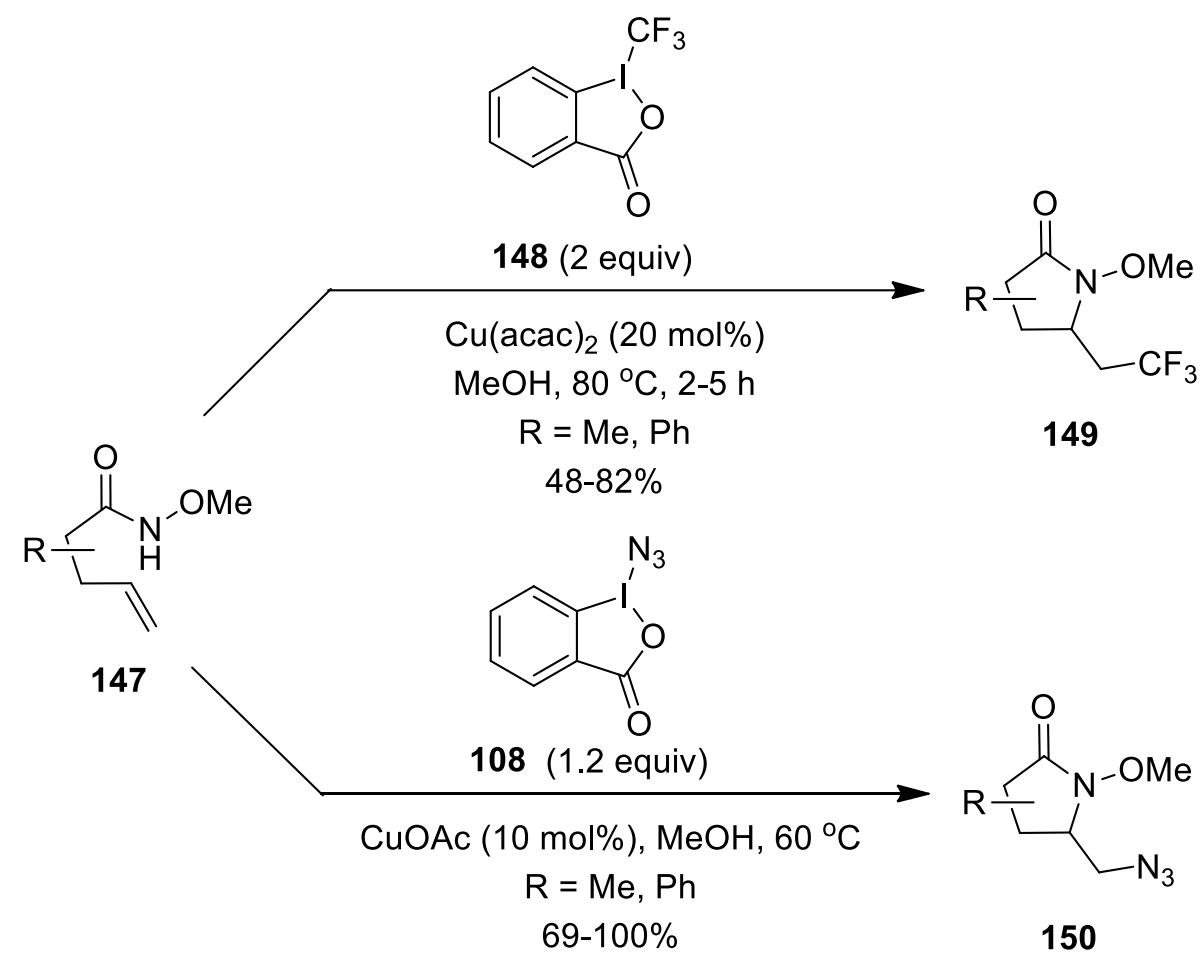

Scheme 37. Hypervalent iodine-mediated synthesis of lactams 149 and 150.

Further, Borelli et al. employed PIFA 120 as an oxidant in the cyclization of allyl or crotyl $N$-sulfonyl-amides 151 to yield 2-propenylimidazolidinones 153 (Scheme 38). ${ }^{74}$ The proposed reaction mechanism possibly involves oxidation of $\mathrm{Pd}(\mathrm{OAc})_{2}$ by PIFA 120 to generate $\mathrm{Pd}\left(\mathrm{O}_{2} \mathrm{CCF}_{3}\right)_{4}$ in situ, which initiates allylic $\mathrm{C}-\mathrm{H}$ activation to form $\eta^{3}$-allylcomplex 152 and subsequent intramolecular cyclization gives cyclic product 153. 


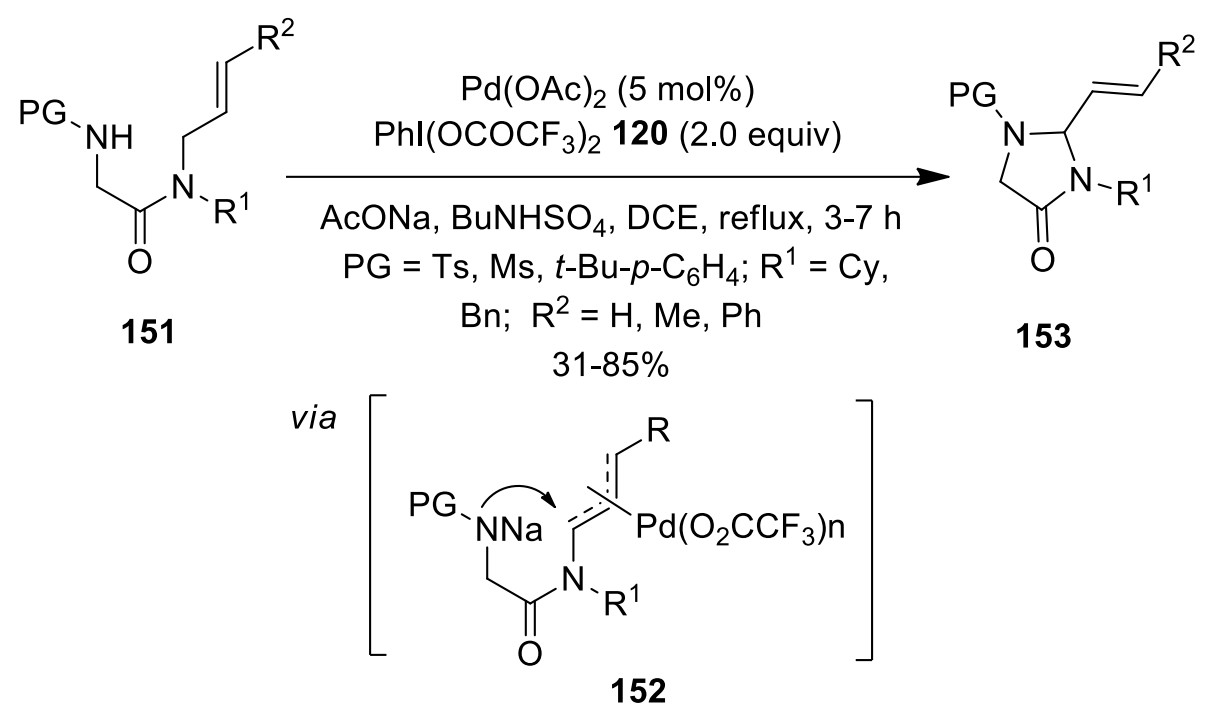

Scheme 38. Synthesis of 2-propenylimidazolidinones 153 using PIFA 120 as an oxidant.

2.1.2.8. Synthesis of thiazoles and thiadiazoles. An inter-/intra-molecular thioamination of $N$-allylthioamides 154 has been demonstrated by Hong and co-workers (Scheme 39). This reaction system featured the use of bistosylimide 46 as the nitrogen source and PIDA 6 as the oxidant to deliver 5-amino-thiazolines 155 via 5-exo cyclization in useful yields. ${ }^{48}$

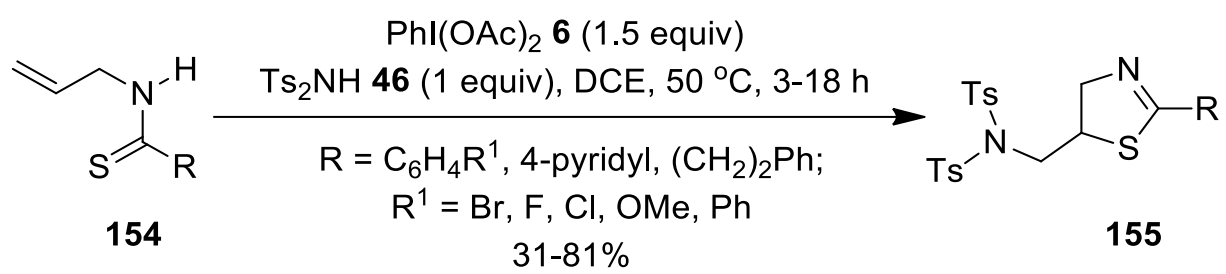

Scheme 39. PIDA-mediated synthesis of oxazolines 155 from $N$-allylthioamides 154.

Further, Han et al. reported catalytic oxidative coupling of thiosemicarbazide $\mathbf{1 5 7}$ mediated by in situ generated PIDA 6 from PhI 13 in the presence of external oxidant $\mathrm{H}_{2} \mathrm{O}_{2}$ (Scheme 40). ${ }^{75}$ This protocol provides an easy access to the biologically important 2-amino-1,3,4-thiadizoles 159 in moderate to high yields. Aromatic, heteroaromatic and alkyl aldehydes were well tolerated.

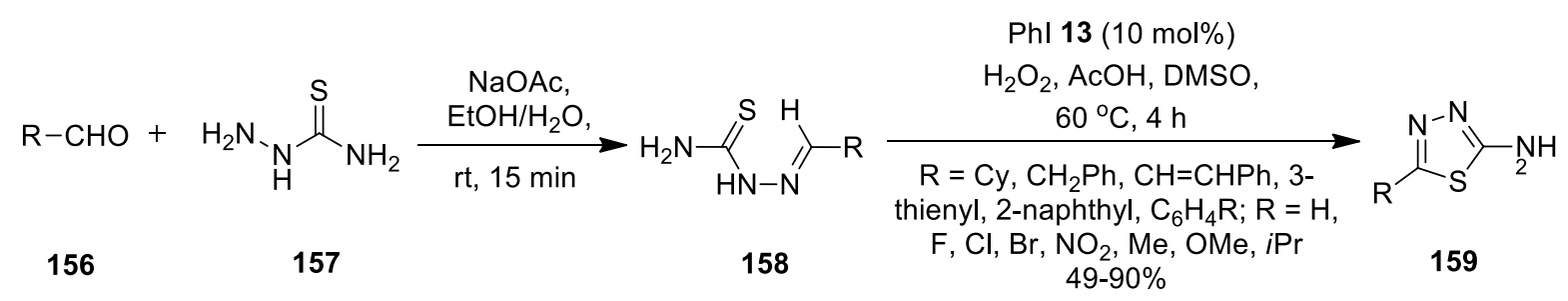

Scheme 40. Synthesis of 2-amino-1,3,4-thiadizoles 159 using iodobenzene 13 as precatalyst. 
A plausible mechanism for the synthesis of 2-amino-1,3,4-thiadizoles 159 is depicted in scheme 41. Initially, $\mathrm{H}_{2} \mathrm{O}_{2}$ oxidizes $\mathrm{Phl} 13$ to generate $\mathrm{Phl}(\mathrm{OAc})_{2} 6$ in situ, which reacts with 158 to give intermediate 160. Cationic species 161 formed through intramolecular cyclization of intermediate 160, losses hydrogen ions to deliver desired product 159.

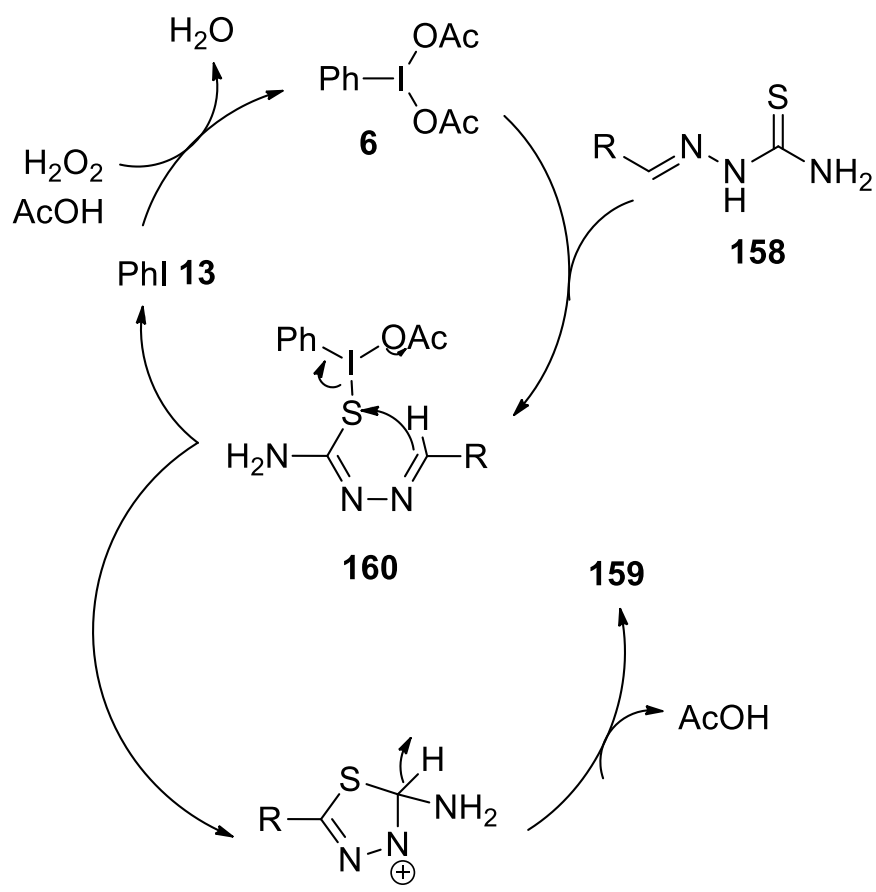

161

Scheme 41. The proposed mechanism for the synthesis of 2-amino-1,3,4-thiadizoles 159 using iodobenzene 13 as precatalyst.

2.1.3. Synthesis of six/seven-membered heterocycles. In 2015, Broggini et al. performed Pd(II)-catalyzed interintramolecular aminoacetoxylation of glycine allylamides 162 to prepare 5-acetoxymethyl-substituted piperazinones 163 employing $\mathrm{Phl}(\mathrm{OAc})_{2} 6$ as the oxidizing agent (Scheme 42). ${ }^{76}$ Reaction initiates with the formation of $\sigma$-alkyl Pd-complex 164 through $\mathrm{Pd}(\mathrm{II})$-mediated aminopalladation process, which is further oxidized to alkyl-Pd(IV) intermediate 165 by $\mathrm{PhI}(\mathrm{OAc})_{2}$ 6. Finally, intermediate 165 undergoes reductive elimination via $\mathrm{C}-\mathrm{O}$ bond formation to yield desired product $\mathbf{1 6 3}$ with the regeneration of catalyst. 


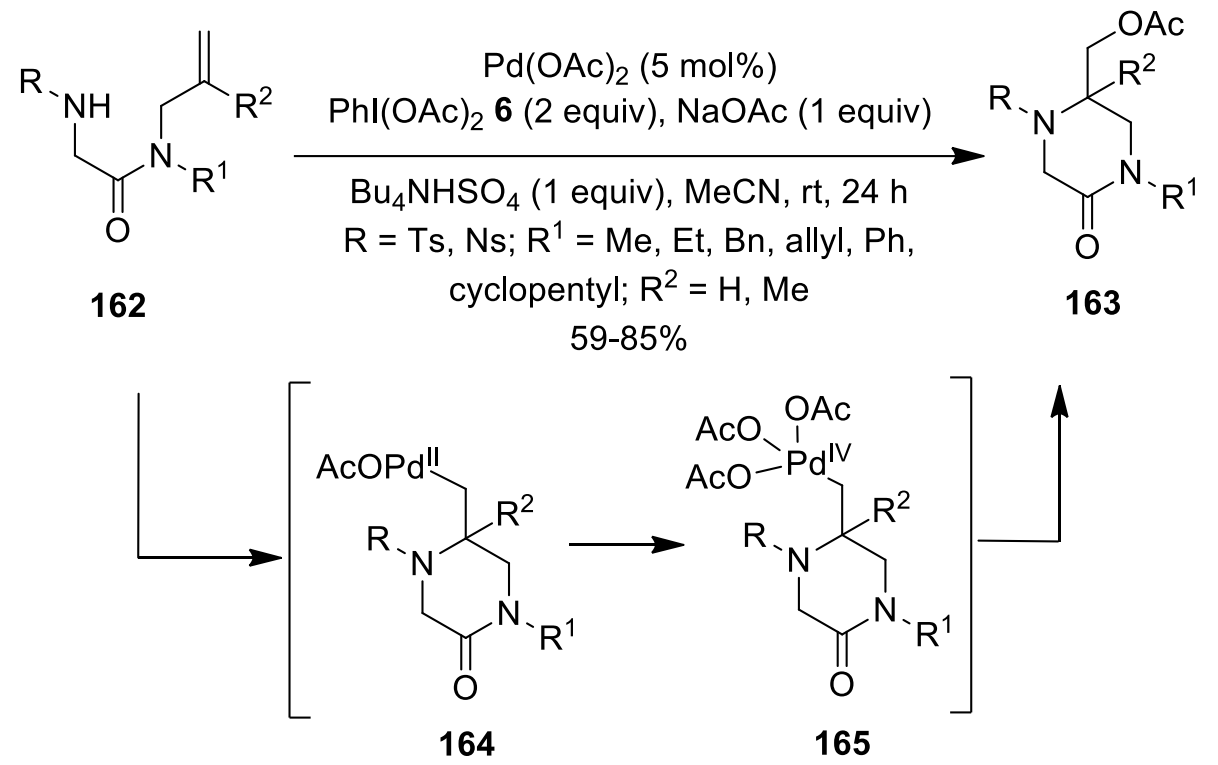

Scheme 42. Synthesis of 5-acetoxymethyl-substituted piperazinones 163 using $\mathrm{Phl}(\mathrm{OAc})_{2} 6$ as an oxidant.

An elegant method featuring PIDA-mediated halocyclization of $S$-alkenylsulfoximines $\mathbf{1 6 6}$ was developed by Bolm's research group (Scheme 43). ${ }^{77}$ Among the various iodine sources screened, potassium iodide provided the best result. The present intramolecular iodoamination process enabled synthesis of tetrahydro-1,2-thiazine1-oxides 167 in variable yields with remarkable regioselectivities and diastereoselectivities. However, substrates with tri-substituted double bonds were unsuitable for this cyclization reaction. Additionally, preparation of dihydro isothiazoles was achieved in 69-90\% yields with high $d r$ (71:29-80:20) under identical conditions.

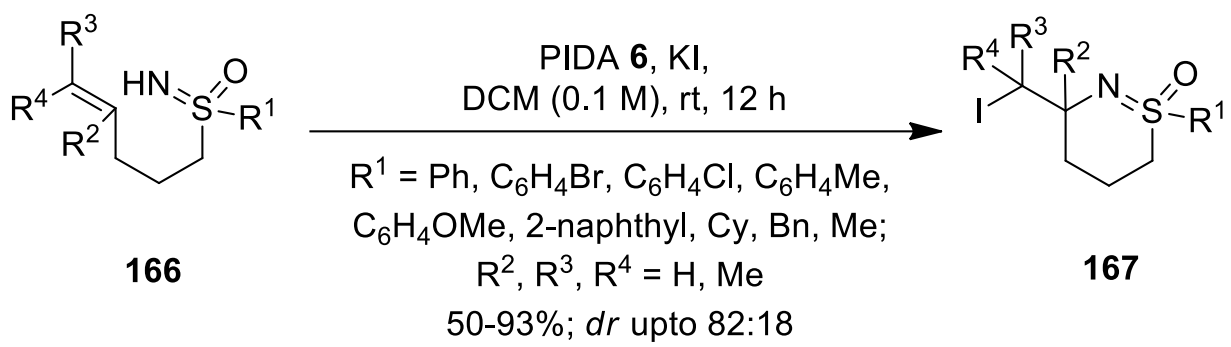

Scheme 43. Synthesis of tetrahydro-1,2-thiazine-1-oxides 167 using oxidant PIDA 6.

In 2016, an intramolecular cyclization of $N$-(E)-alkenylamides 168 to the corresponding 6-aryl-5-acetoxy-2oxazines 169 induced by PIDA 6 was described by Ranjith et al. (Scheme 44). ${ }^{44}$ In the proposed mechanism, aryliodinium ion $\mathbf{3 8}$ formed from PIDA 6 and HF.py interacts with the alkene $\mathbf{1 6 8}$ and generates cyclic iodonium ion $\mathbf{1 7 0}$ which is attacked by the amide moiety to give alkyl iodane 171. Notably, the presence of aryl group at the end of the alkene stabilizes the incipient carbocation thereby facilitating endo-cyclization of intermediate 171. Finally, nucleophilic attack by the acetyl group via $S_{N} 2$-like bimolecular reductive elimination furnishes desired oxazine 169 and releases Phl 13. 


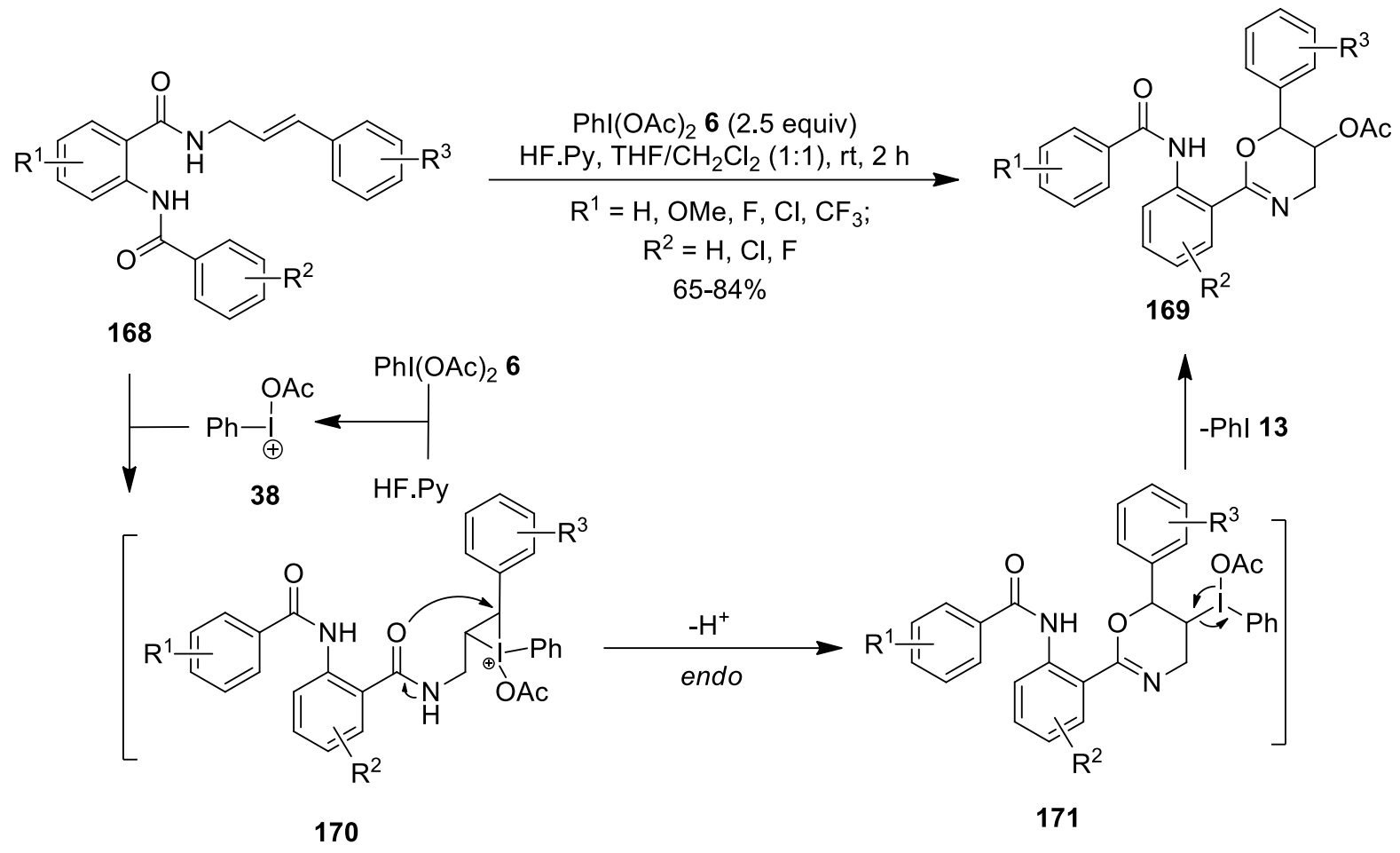

Scheme 44. Synthesis of 6-aryl-5-acetoxy-2-oxazines 169 using PIDA 6 as an oxidant.

Later, Borelli et al. reported Pd-catalyzed intramolecular cyclization of $\mathrm{N}$-sulfonyl- $\mathrm{N}^{\prime \prime}$-crotyl-benzylamides 162 via aminopalladation/dehydropalladation process using terminal oxidant $\mathrm{Phl}\left(\mathrm{O}_{2} \mathrm{CCH}_{3}\right)_{2} 6$ (Scheme 45). ${ }^{74}$ The reaction was carried out in the presence of $\mathrm{AcONa}$ and $\mathrm{Bu}_{4} \mathrm{NHSO}_{4}$ in DCE under refluxing conditions. The expected product vinyl piperazinones $\mathbf{1 7 2}$ were obtained in moderate yields.

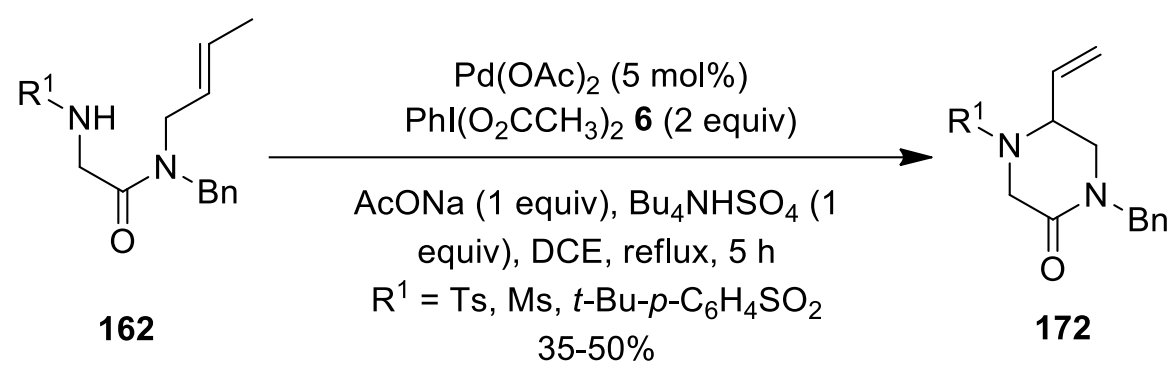

Scheme 45. Synthesis of vinyl piperazinones 172 using PIDA 6 as an oxidant.

Wengryniuk's research group prepared six or seven membered cyclic ethers 175 by employing (poly)cationic $\lambda^{3}$-iodane ( $N$-HVI) 174 as electrophilic reagent for the activation of secondary alcohols 173 (Scheme 46). ${ }^{78}$ Presence of $\mathrm{N}$-HVI 174 was crucial for the excellent selectivity achieved for $\mathrm{C}-\mathrm{O}$ bond migration over direct oxidation via $\alpha$-elimination pathways. Additionally, ring expansion strategy was successfully applied in the latestage derivatization of several natural products. Further synthesized HFIP-acetals could be easily derivatized with different nucleophiles, providing scope for subsequent manipulations. 


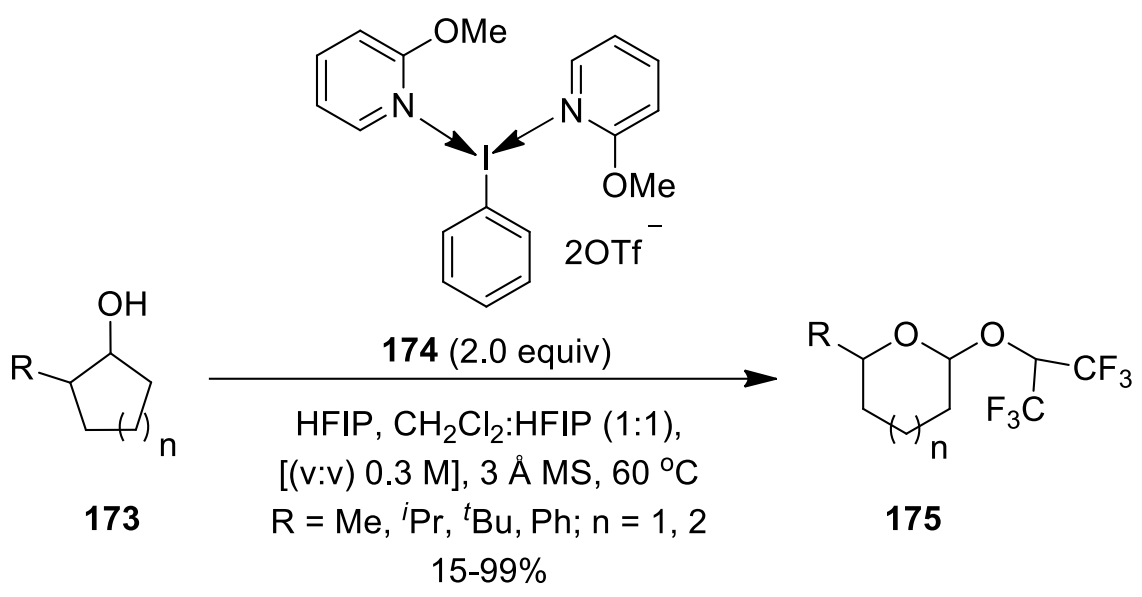

Scheme 46. Synthesis of six or seven membered cyclic ethers 175 using (poly)cationic $\lambda^{3}$-iodanes 174.

\subsection{Synthesis of bicyclic heterocycles}

In recent years, various intra- and inter-molecular approaches have been developed for the preparation of bicyclic heterocycles. Most of these reactions require stoichiometric hypervalent iodine reagents as oxidants while few employ chiral/achiral aryl iodides as precatalysts. In this section, all hypervalent-mediated or catalysed reactions for the construction of bicyclic heterocycles will be discussed.

The group of Gaunt has designed a novel $\mathrm{C}-\mathrm{H}$ activation strategy for the transformation of aliphatic secondary amines $\mathbf{1 7 6}$ possessing adjacent methyl group into the corresponding bicyclic heterocycles $\mathbf{1 7 7}$ using $\mathrm{Pd}(\mathrm{OAc})_{2} / \mathrm{PhI}(\mathrm{OAc})_{2} 6$ catalytic system (Scheme 47). ${ }^{79}$ This $\mathrm{C}-\mathrm{H}$ aziridination process proceeds via $\mathrm{Pd}(\mathrm{IV})$ intermediate 178 which upon subsequent $\mathrm{C}-\mathrm{N}$ bond reductive elimination delivers aziridines 177 . In continuation, the same team prepared azetidines 181 via $\mathrm{Pd}$-catalyzed intramolecular $\gamma-\mathrm{C}-\mathrm{H}$ amination of substituted morpholinones $\mathbf{1 7 9}$ containing $\alpha$-ethyl group. ${ }^{80}$ Presence of the oxidant benziodoxole tosylate $\mathbf{1 8 0}$ with additive $\mathrm{AgOAc}$ played a crucial role in controlling selective $\mathrm{C}-\mathrm{N}$ reductive elimination pathway leading to azetidines 181. The present protocol tolerated range of substituents, including enantio-enriched substrates which yield chiral azetidines $\mathbf{1 8 1}$ with excellent diastereoselectivity. Interestingly, substrates $\mathbf{1 7 9}$ possessing C$\mathrm{H}$ bond at the $\alpha$-position to the amine were well tolerated, unlike in the previous developed protocol. ${ }^{79}$ 

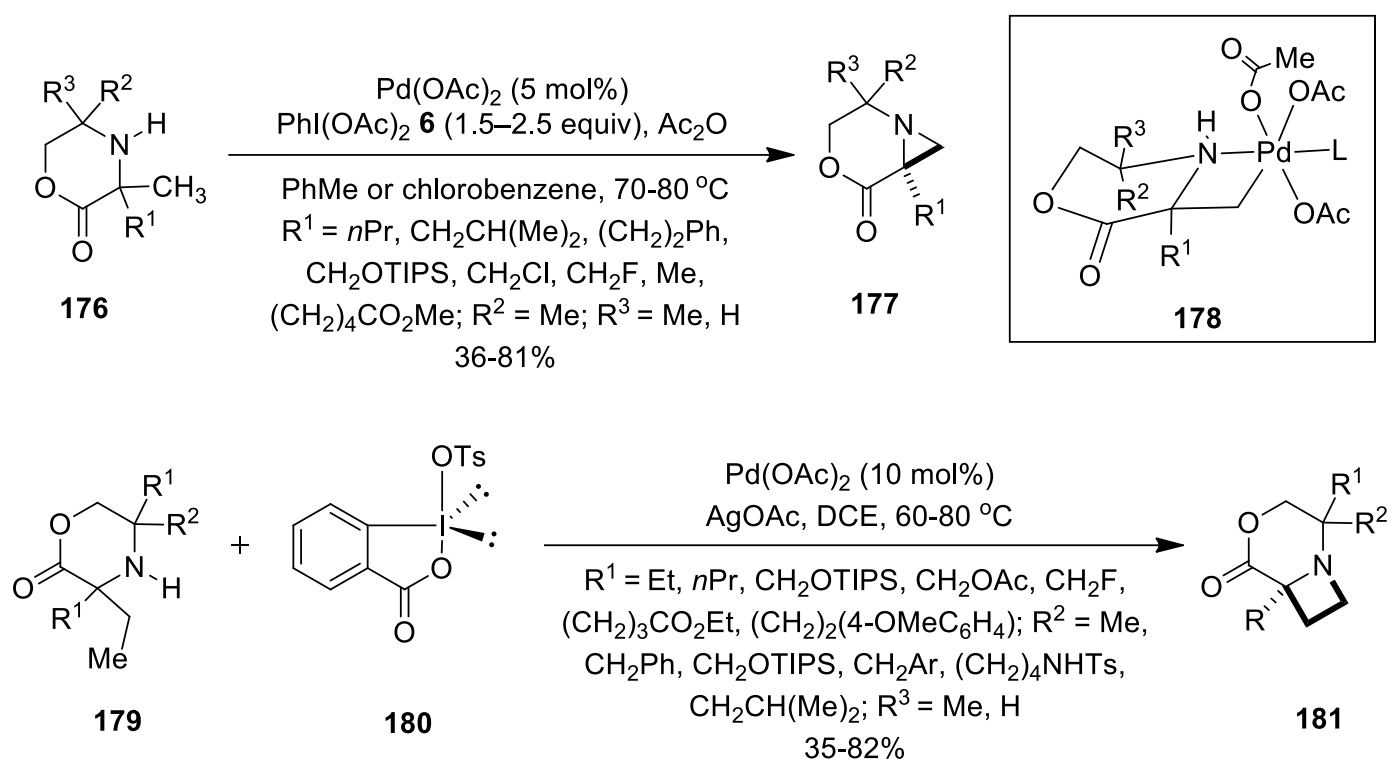

Scheme 47. Synthesis of fused aziridines 177 and azetidines 181 via C-H activation strategy.

Murphy's group reported preparation of dihydrofurans 184 by reacting electron-rich styrenes 182 with cyclic iodonium ylides $\mathbf{1 8 3}$ (Scheme 48). ${ }^{81}$ The reaction was mediated by $\mathrm{Phl}_{(\mathrm{OAc})_{2}} \mathbf{6}$ in the presence of $\mathrm{Bu}_{4} \mathrm{NI}$ as an iodine source. Though only few examples were reported, the present method provided bicyclic products 184 in significant yields.

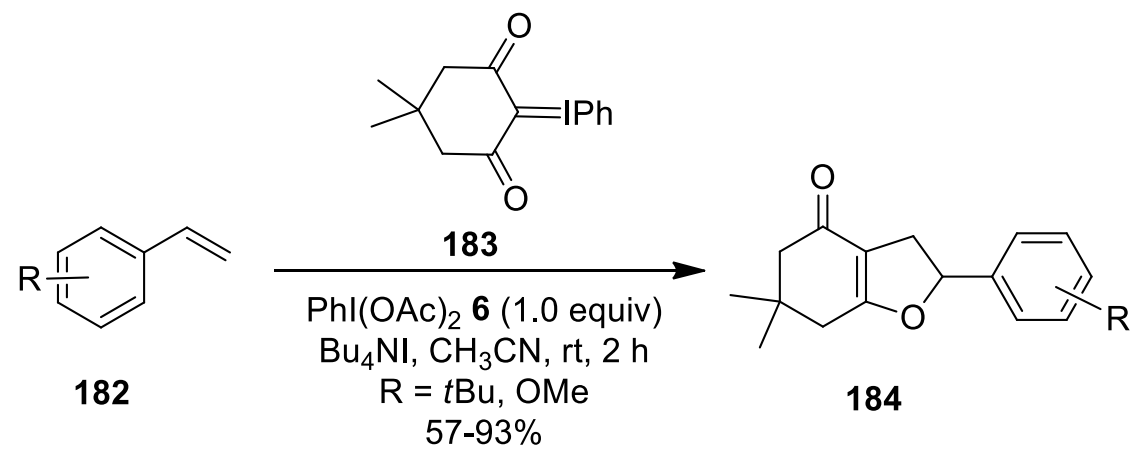

Scheme 48. Synthesis of dihydrofurans 184 by reacting iodonium ylides 183 with styrenes 182 .

An efficient catalytic protocol featuring hypervalent iodine(III)-induced oxidative cycloaddition of various aldoximes $\mathbf{5 7}$ with maleimides $\mathbf{1 8 5}$ to prepare pyrrolo-isoxazolines $\mathbf{1 8 6}$ was described by Yoshimura et al. (Scheme 49). ${ }^{82}$ This cyclization reaction involves in situ generation of hydroxy(aryl)iodonium species (IBA-OTf) 75 from corresponding 2-iodobenzoic acid 76 in the presence of $m$ CPBA and TfOH. The proposed mechanism is similar to that discussed in Scheme 19 wherein oxidation of aldoximes generates nitrile oxide which later undergoes cycloaddition with $\mathbf{1 8 5}$ to deliver product 186. 


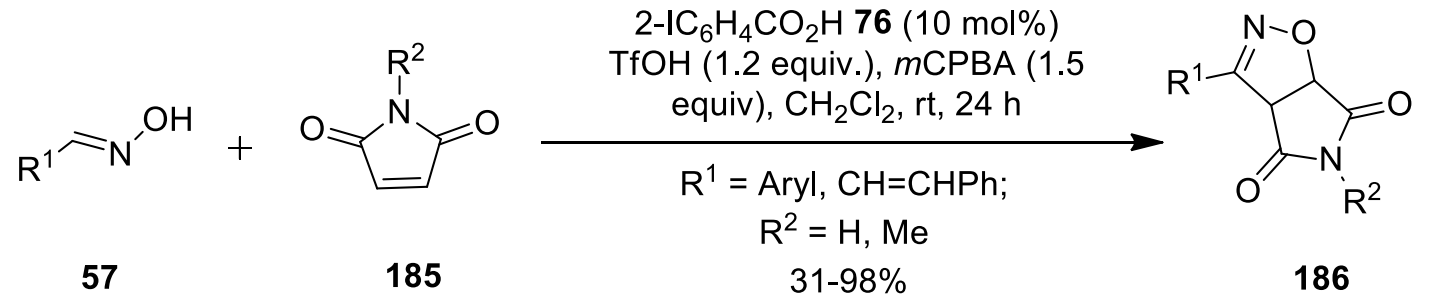

Scheme 49. Synthesis of pyrrolo-isoxazolines 186 using precatalyst 2-iodobenzoic acid 76.

Later, the same group reported oxidative heterocyclization of aldoximes $\mathbf{5 7}$ with 1-propene-1,3-sultone $\mathbf{1 8 8}$ mediated by Koser's reagent 187 furnishing isoxazoline-ring-fused heterobicyclic products $\mathbf{1 8 9}$ (Scheme 50). ${ }^{83}$ Furthermore, reaction of aldoximes $\mathbf{5 7}$ with 3-methyl-1-phenyl-2-phospholene-1-oxide 190 enabled synthesis of isoxazoline-fused phospholene oxides 191 under identical conditions. The proposed mechanism involves Koser's reagent-induced oxidation of aldoximes $\mathbf{5 7}$ to generate nitrile oxides $\mathbf{7 9}$ in situ, which undergoes subsequent intermolecular 1,3-dipolar cycloaddition with heterocyclic alkenes to deliver respective heterobicyclic products.

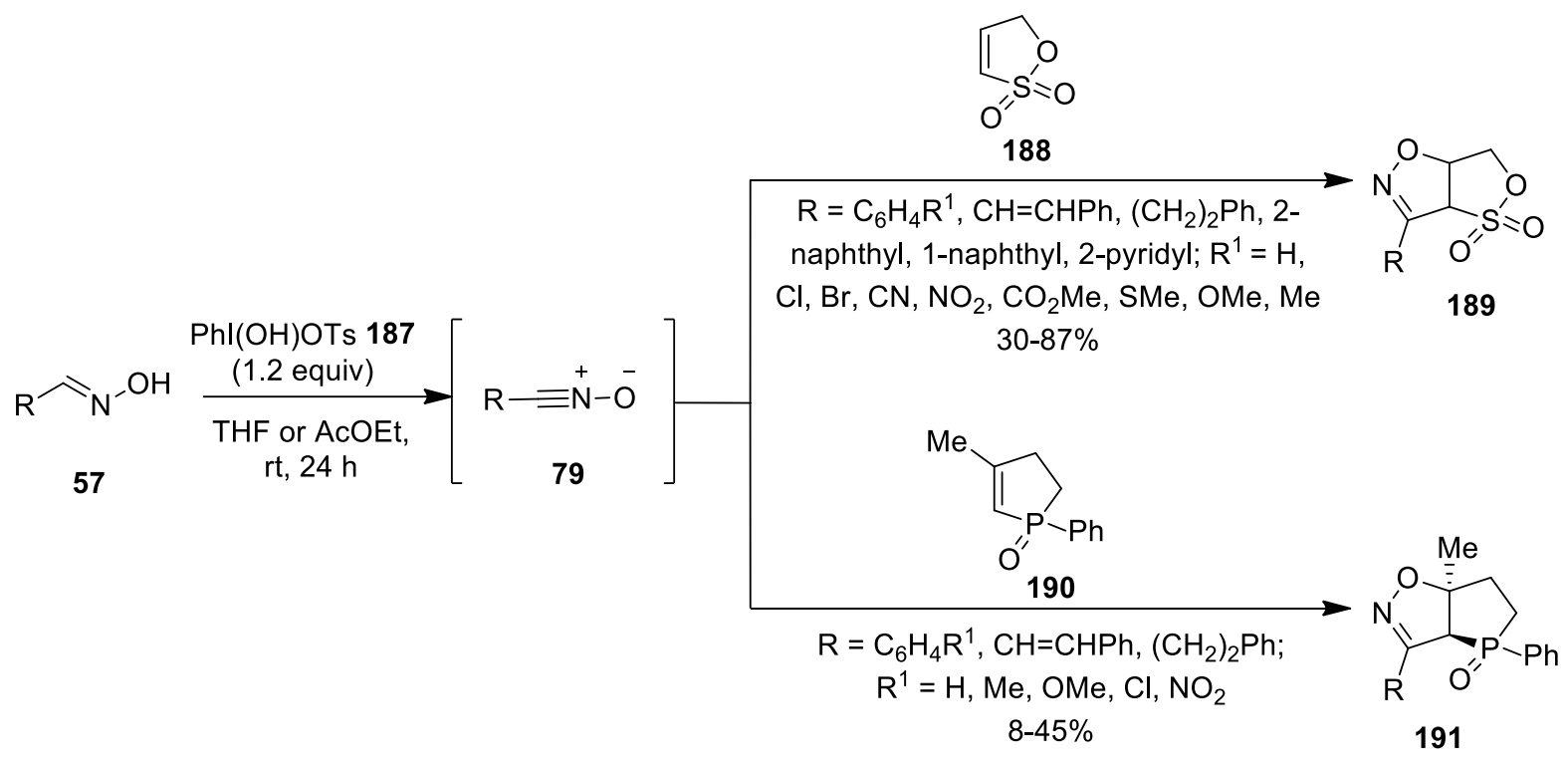

Scheme 50. Synthesis of heterobicyclic products 189 and 191 using Koser's reagent 187 as an oxidant.

In 2019, Tong's group performed a PIDA-induced intramolecular acetoxylative $(3+2)$ cycloaddition of 1,6enynes 192 in a 6-exo manner via $\mathrm{Pd}(\mathrm{II})-\mathrm{Pd}(\mathrm{IV})$ catalysis ${ }^{84}$ This cyclization reaction afforded bicyclic heterocycles 193 in variable yields (Scheme 51). The proposed mechanism begins with the formation of alkenyl-Pd(II) intermediate 194 via alkyne acetoxypalladation process, following which alkene insertion occurs to form alkyl$\mathrm{Pd}(\mathrm{II})$ intermediate 196 through chair-like transition state 195. Further 196 is oxidized to bicyclic $\mathrm{Pd}(\mathrm{IV})$ intermediate 197 using oxidant $\mathrm{Phl}(\mathrm{OAc})_{2}$ 6, which gives cyclometalated alkoxyPd(IV)-alkyl intermediate 198 with the loss of $\mathrm{AcOH}$. Finally, direct $\mathrm{C}-\mathrm{O}$ reductive elimination of 198 delivers product 193 and regenerate palladium catalyst. 


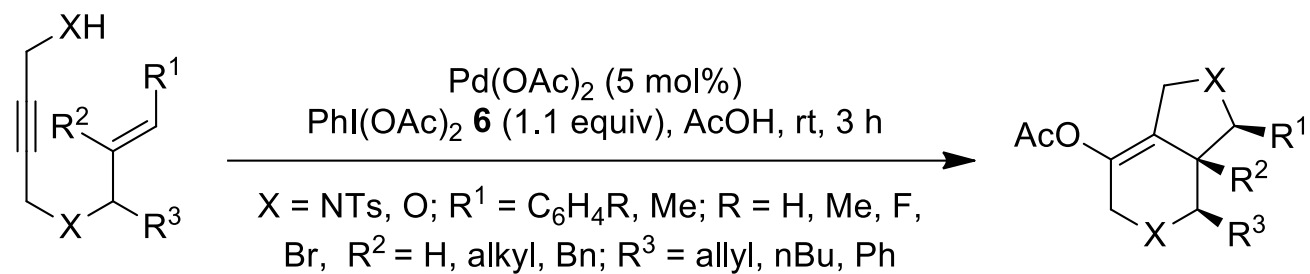

192

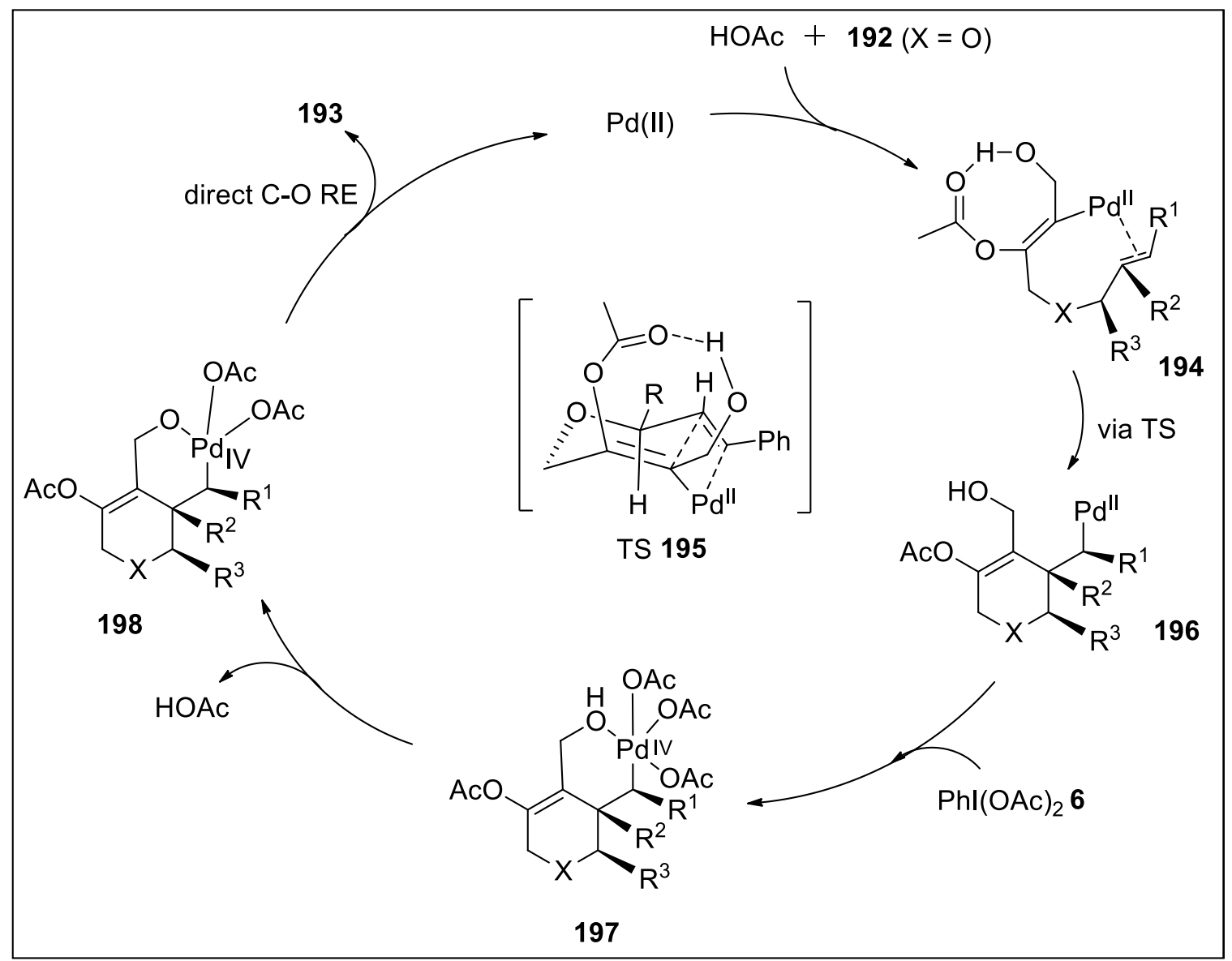

Scheme 51. Synthesis of bicyclic heterocycles 193 using oxidant $\mathrm{PhI}(\mathrm{OAc})_{2} 6$.

Further, when the ligand 1,10-phenanthroline was introduced, 1,6-enynes 192 were converted into 3bicyclo[4.1.0]-heptan-5-one products 200 via ligated Pd(IV) intermediate 199 (Scheme 52). ${ }^{84}$ The presence of the additional coordinating ligand for $\mathrm{Pd}(\mathrm{IV})$ obstruct the direct $\mathrm{C}-\mathrm{O}$ bond reductive elimination and promotes reaction via a $\mathrm{S}_{\mathrm{N}} 2$-type $\mathrm{C}-\mathrm{C}$ reductive elimination pathway. 


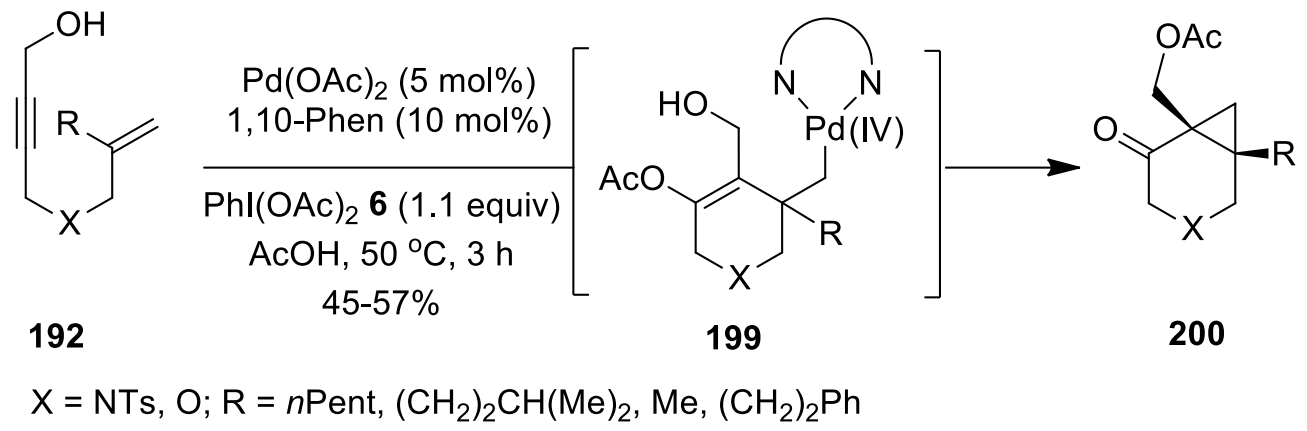

Scheme 52. Synthesis of 3-bicyclo[4.1.0]-heptan-5-one products 200 using oxidant Phl(OAc) 6 .

The synthesis of benzo-fused heterocycles has been well studied using different hypervalent iodine reagents. Further in this section, we will be discussing the synthesis of variety of heterocyclic compounds in which benzene ring is fused with five-, six- and seven-membered heterocycles in briefly. In 2017, Bedford et al. performed intramolecular benzylic C-H sulfamidation of 2-benzyl- $N$-sulfonylbenzamide substrates 201 catalysed by $\mathrm{Cu}(\mathrm{OTf})_{2}$ in the presence of PIDA 6 as the terminal oxidant (Scheme 53). ${ }^{85}$ The present method leads to the synthesis of $\mathrm{N}$-arylsulfonyl-1-arylisoindolinones 202 in useful yields. Interestingly, sulfonamide moiety behaves as directing group as well as functionalizing reagent in this reaction. Further samarium iodidemediated deprotection of $\mathbf{2 0 2}$ provides valuable free 1-arylisoindolinone.

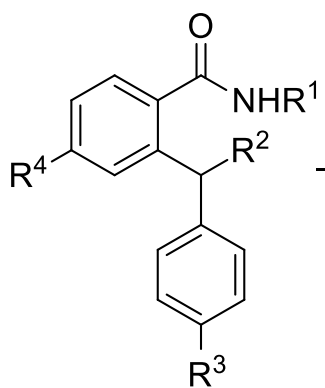

201

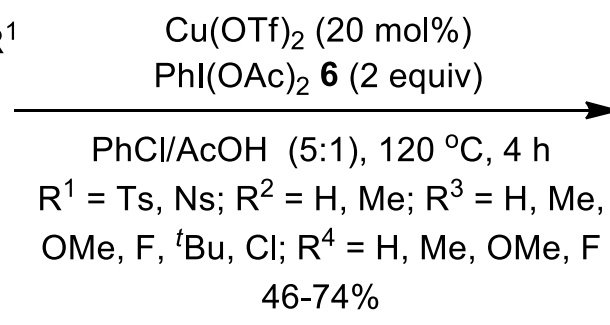

46-74\%

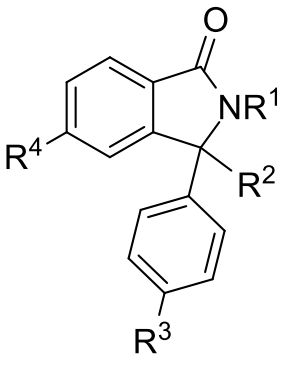

202

Scheme 53. Synthesis of $\mathrm{N}$-arylsulfonyl-1-arylisoindolinones 202 using PIDA 6 as the terminal oxidant.

In the same year, an elegant catalytic strategy to prepare biologically important scaffolds indolizines $\mathbf{2 0 8}$ ( $X$ $=\mathrm{C})$ and imidazopyridines $208(\mathrm{X}=\mathrm{N})$ was developed by Wang and co-workers (Scheme 54). ${ }^{86}$ This transformation took place via Michael addition-[3 +2] annulation of 2-substitued azaarenes 203 and $\alpha, \beta-$ unsaturated aldehydes 204. The reactions are promoted by amine catalyst 205 and $\mathrm{N}$-heterocyclic carbene (NHC) 207 relay catalysis in the presence of oxidant PIDA 6 and base DMAP. Notably, preformation of the Michael adduct 206 from 203 and 204 was necessary which could be used without further purification. Furthermore, preparative power of this method was demonstrated for synthesizing an anxiolytic drug, Saripidem in $45 \%$ yield. 


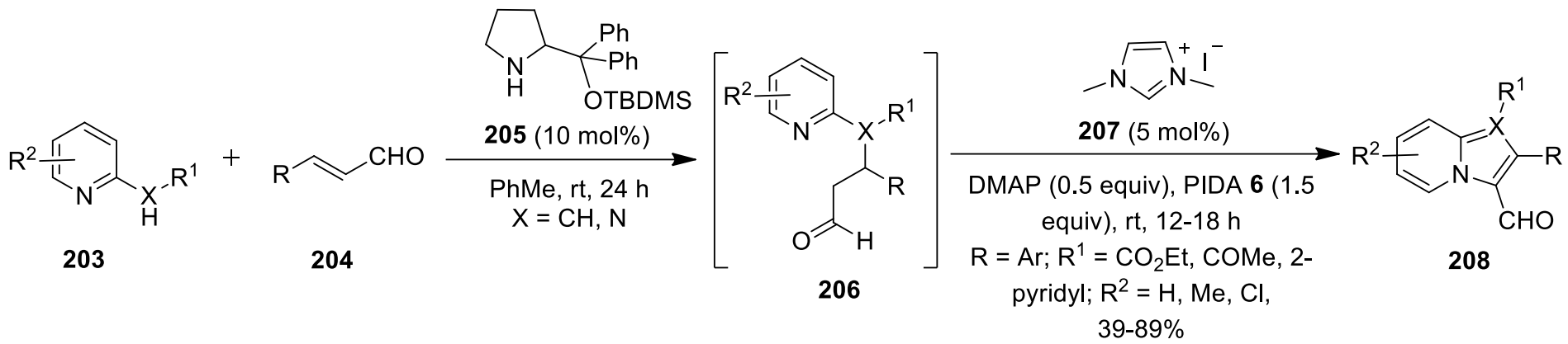

Scheme 54. Synthesis of indolizines and imidazopyridines 208 using PIDA 6 as an oxidant.

Later, Miki's research team described a concise route to access 3-acylindole derivatives $\mathbf{2 1 2}$ by performing PIDA-mediated oxidative rearrangement of 2-aminochalcones $\mathbf{2 0 9}$ to form acetal intermediate $\mathbf{2 1 1}$ (Scheme 55). ${ }^{87}$ Subsequent treatment with $\mathrm{K}_{2} \mathrm{CO}_{3}$ at room temperature resulted in 3-acylindoles 212 via intramolecular cyclization process. Chalcones 209 bearing substituted phenyl, thiophene and alkyl groups were well tolerated. Furthermore, scope of this method was extended towards the rapid synthesis of SCB01A, currently evaluated as a potential anticancer drug.

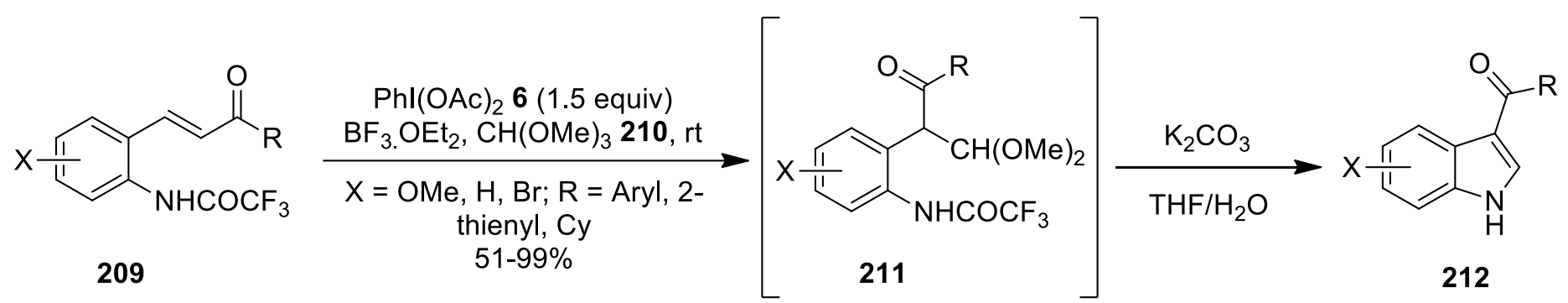

Scheme 55. PIDA-mediated synthesis of 3-acylindoles 212.

The proposed mechanism for the synthesis of 3-acylindoles $\mathbf{2 1 2}$ is depicted in scheme 56 . Reaction begins with the electrophilic addition of PIDA 6 to the double bond alkene 209 mediated by $\mathrm{BF}_{3} \cdot \mathrm{OEt}_{2}$ and $\mathrm{MeOH}$ to form adduct 213. Subsequent oxidative rearrangement assisted by the lone pair of oxygen causes aryl group migration to yield oxonium intermediate 214, which could be converted into corresponding acetal 211 in the

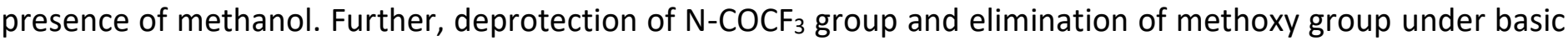
conditions furnishes intermediate $\mathbf{2 1 5}$ which then undergo subsequent cyclization and aromatization to deliver anticipated product 212 . 


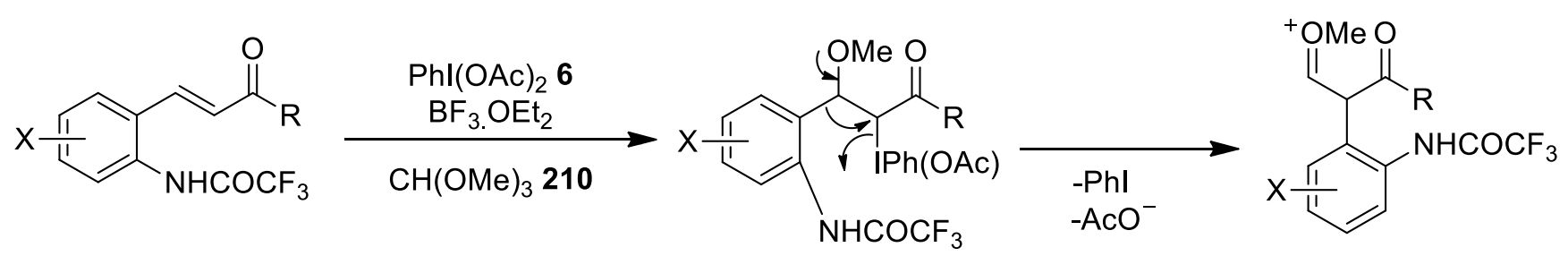

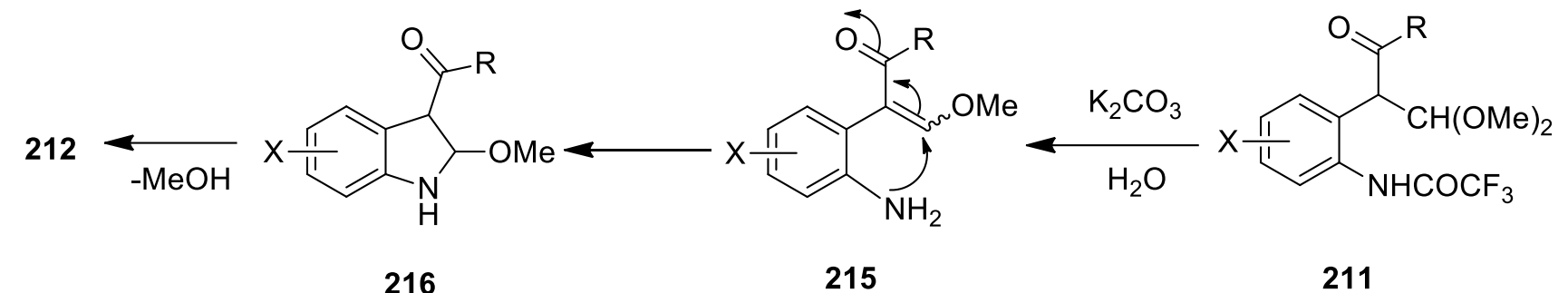

Scheme 56. The proposed mechanism for the PIDA-mediated synthesis of 3-acylindoles 212.

In 2018, Xia et al. synthesized a new water-soluble and highly acidic hypervalent iodine(III) reagent, (phenyliodonio)sulfamate (PISA) 218 by reacting $\mathrm{PhI}(\mathrm{OAC})_{2} 6$ with $\mathrm{NH}_{2} \mathrm{SO}_{3} \mathrm{H}$ in $\mathrm{MeCN}$ at room temperature. ${ }^{88}$ Using PISA, synthesis of various substituted indoles 219 from 2-alkenylanilines 217 involving aryl migration/intramolecular C-H cyclization cascade process was demonstrated successfully (Scheme 57). PISA 218 behaves as both oxidant and lewis acid in this reaction. Further developed methodology has been utilized for the synthesis of the bioactive molecule Pravadoline and anti-inflammatory drug molecules such as Indometacin and Zidometacin.

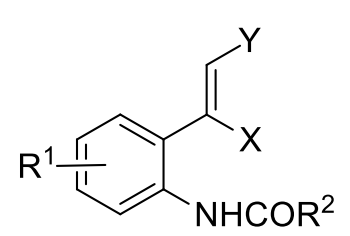

217

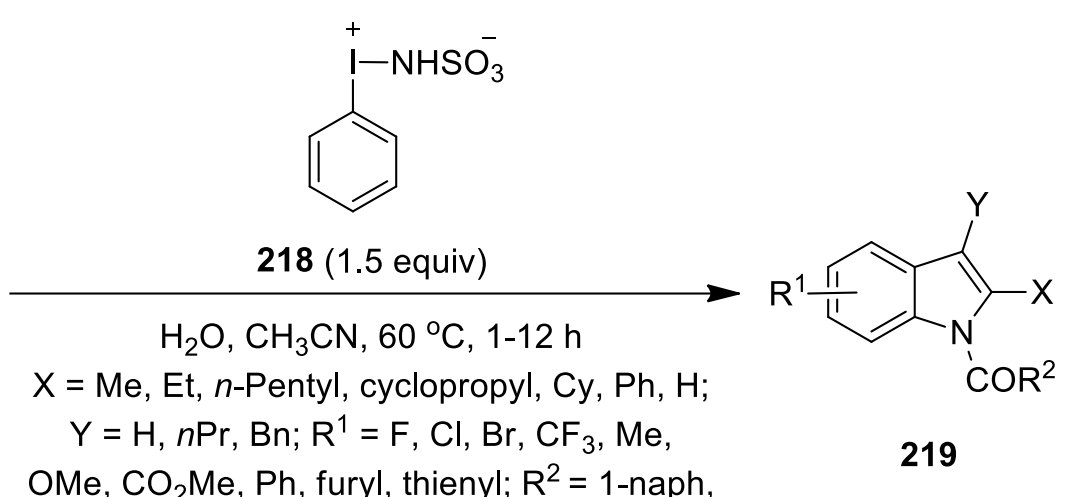

2-thienyl, 2-furyl, Bn, Me, vinyl

$57-88 \%$

Scheme 57. Synthesis of indoles 219 using (phenyliodonio)sulfamate 218 as an oxidant.

Further for the synthesis of 1,2-disubstituted benzimidazoles 221, an intramolecular benzylic $\mathrm{C}\left(\mathrm{sp}^{3}\right)-\mathrm{H}$ imination strategy involving $4-\mathrm{H}$ elimination was designed by Mal's research group. ${ }^{89}$ This method enabled selective functionalization of two aliphatic- $\mathrm{C}\left(s p^{3}\right) \mathrm{H}$ and two aryl- $\mathrm{N}\left(s p^{3}\right) \mathrm{H}$ at 1,5 position facilitated by in situ 
generated hypervalent iodine(III) species from PhI-mCPBA catalytic system (Scheme 58). Later, the same group developed another catalytic route employing precatalyst tetrabutylammonium iodide $\mathbf{2 2 2}$ in combination with $t$-BuOOH in DMSO as relatively inexpensive replacement for the previously designed PhI- $m$ CPBA-HFIP system. ${ }^{90}$ Symmetrical dibenzylamines $\mathbf{2 2 0}$ gave single isomer of benzimidazoles while unsymmetrical ones yielded mixture of isomers of imination product under both catalytic conditions.

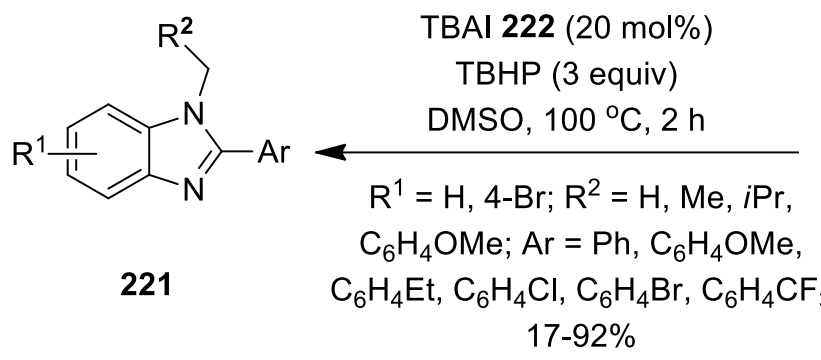

$$
17-92 \%
$$

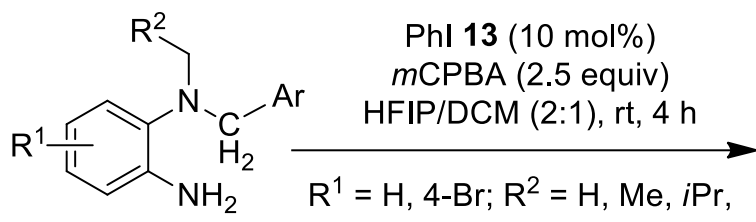

220
$\mathrm{Ph}, \mathrm{C}_{6} \mathrm{H}_{4} \mathrm{OMe} ; \mathrm{Ar}=\mathrm{Ph}$,

$$
\mathrm{C}_{6} \mathrm{H}_{4} \mathrm{OMe}, \mathrm{C}_{6} \mathrm{H}_{4} \mathrm{Cl}, \mathrm{C}_{6} \mathrm{H}_{4} \mathrm{CF}_{3}
$$
$18-92 \%$

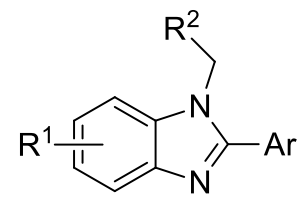

221

Scheme 58. lodine(III)-catalyzed synthesis of 1,2-disubstituted benzimidazoles 221.

Furthermore, Singh and Mangaonkar demonstrated an efficient method for the oxidative cyclization of 2hydroxystilbenes 223 using PhI(OAc) 6 as the catalyst and m-CPBA as the oxidant (Scheme 59)..$^{91}$ This metalfree route gave access to a variety of functionally diverse 2-arylbenzofurans $\mathbf{2 2 4}$ at room temperature. Reaction time was reduced by performing the reaction under ultrasound-irradiation conditions and desired products were obtained in high yields. Very recently, the same group prepared 2-arylbenzofurans 224 by employing PhI 13 (10 mol \%) as precatalyst in the presence of terminal oxidant $m$-CPBA and additive trifluoroacetic acid in $\mathrm{CHCl}_{3} .{ }^{92}$

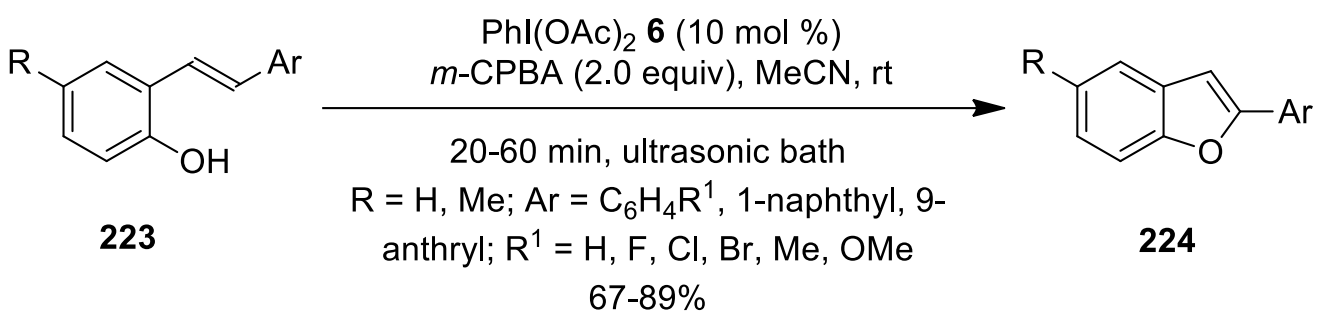

Scheme 59. Synthesis of 2-arylbenzofurans 224 using PIDA 6 as the catalyst.

A plausible catalytic cycle for this cyclization reaction initiates with the activation of double bond of 223 by PIDA 6 to form three-membered iodonium intermediate 225. ${ }^{91}$ Intramolecular cyclization of 225 gives intermediate 226, which upon reductive elimination yields anticipated product 224 with the release of PhI 13. Further PhI 13 could be reoxidized to active iodine(III) species 6 in the presence of $m$ CPBA and acetic acid (Scheme 60). 


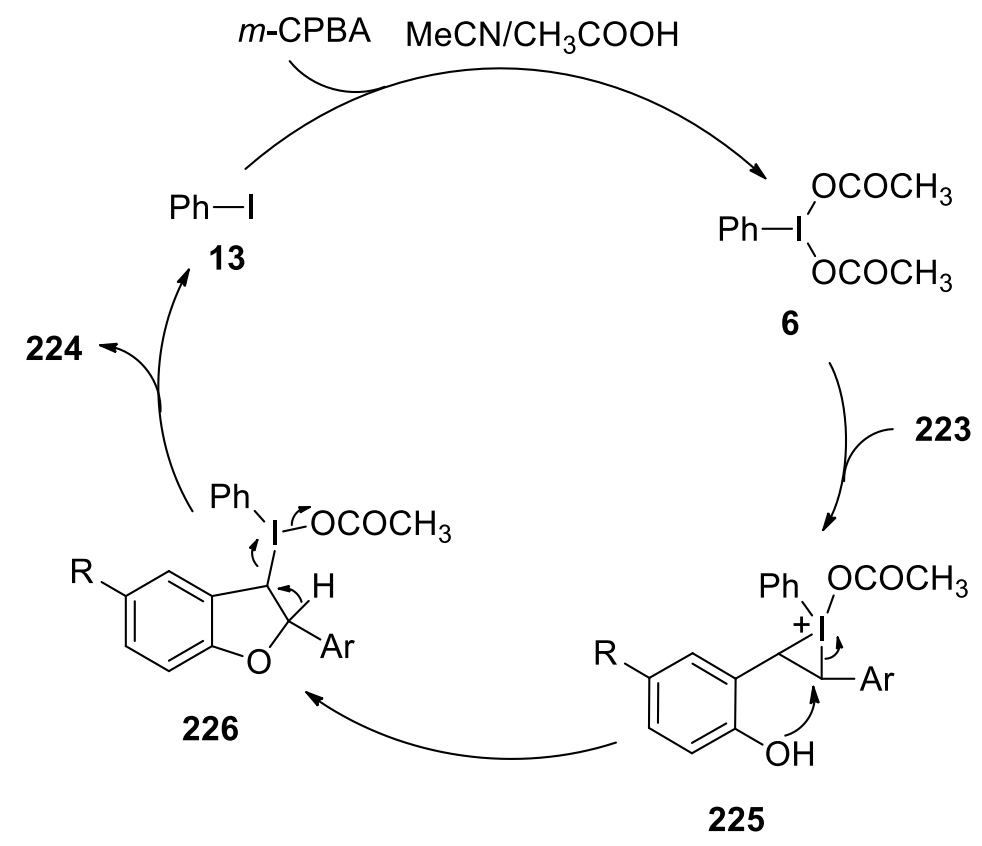

Scheme $\mathbf{6 0}$. The proposed catalytic cycle for the synthesis of 2-arylbenzofurans $\mathbf{2 2 4}$ using catalytic PIDA 6.

In 2019, Cui's research group developed an expedient strategy to prepare quinoxalines 228 from $\mathrm{N}$-(2acetaminophenyl)enaminones $\mathbf{2 2 7}$ via hypervalent iodine(III)-induced intramolecular oxidative $\mathrm{C}-\mathrm{N}$ bond forming tandem process (Scheme 61). ${ }^{93}$ Inspection of various substrates revealed that electron-rich substrates gave desirable product yields while electron-deficient ones provided relatively lower yields. The proposed mechanism initiates with the reaction of 227 with PIDA 6 that generates $\alpha$-iodo iminoketone 229, which undergoes intramolecular condensation cyclization to afford $\mathbf{2 3 0}$ with the release of $\mathrm{Phl} \mathbf{1 3}$ and AcOH. Finally, oxidation of $\mathbf{2 3 0}$ in the presence of oxygen forms 231, which gives final product $\mathbf{2 2 8}$ with the elimination of $\mathrm{CH}_{3} \mathrm{COOH}$. Previously, Zheng and co-workers had constructed quinoxaline scaffolds through Phl(OAc) $)_{2}$-mediated cascade cycloamination of $\mathrm{N}$-aryl ketimines by employing sodium azide as the nitrogen source under copper catalysis. ${ }^{94}$ 


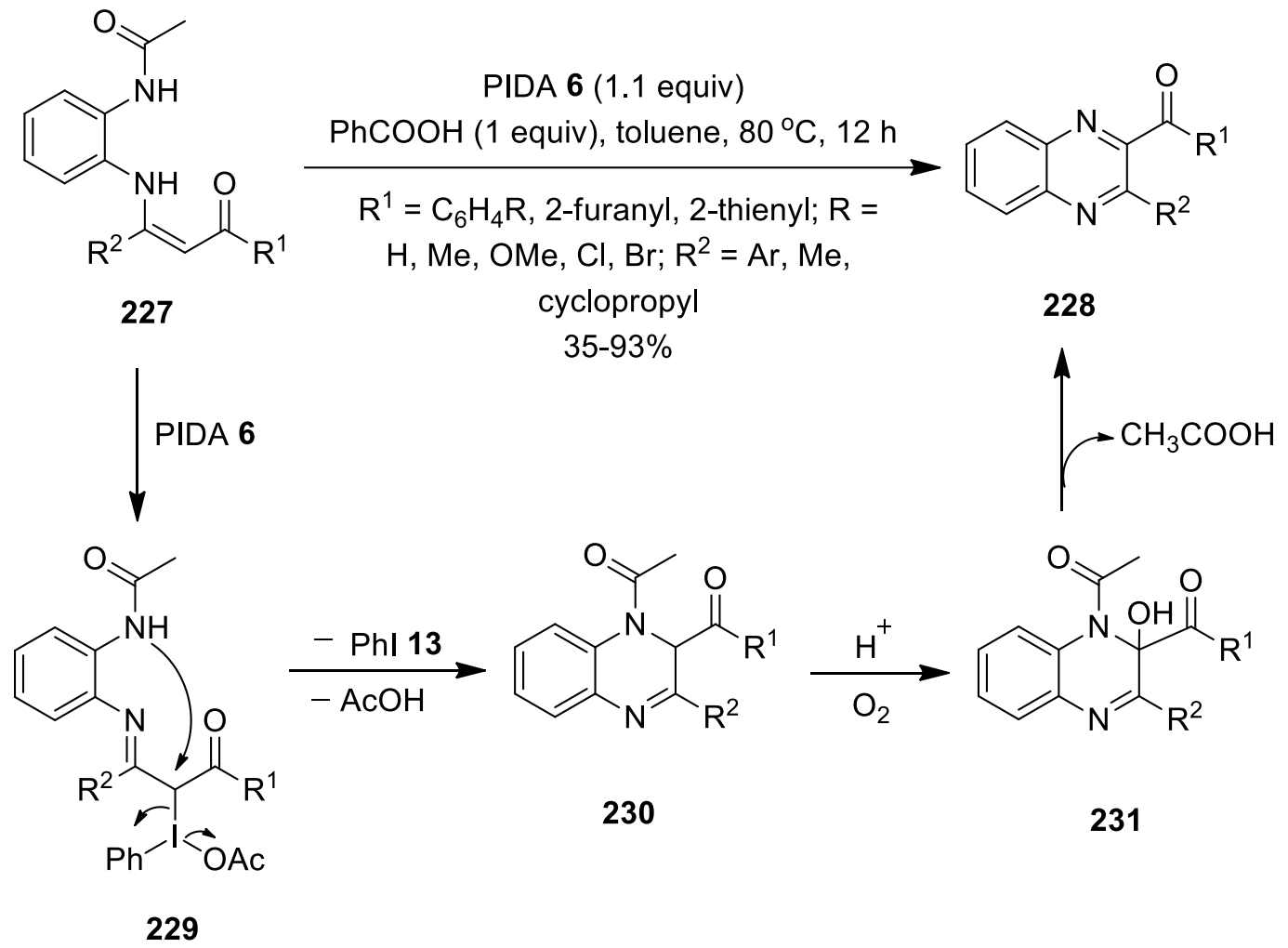

Scheme 61. Synthesis of quinoxalines 228 using PIDA 6 as the oxidant.

Meanwhile, Cai's research group described asymmetric intramolecular $\mathrm{C}-\mathrm{N}$ bond forming reaction of substituted amides 232 via catalytic desymmetrization process (Scheme 62). ${ }^{95}$ This reaction was promoted by in situ generated chiral hypervalent iodine(III) species from diiodospirobiindane derivative $\mathbf{2 3 3}$ in the presence of $m$ CPBA. Addition of TFA as acid promoter and HFIP as solvent media provided the best result. The desired lactams 234 were obtained in decent yields with enantiomeric excess up to 89\%. Notably, cyclopentoxy substituent on the nitrogen of amide gave products with better enantioselectivity than with other alkoxy substituents.

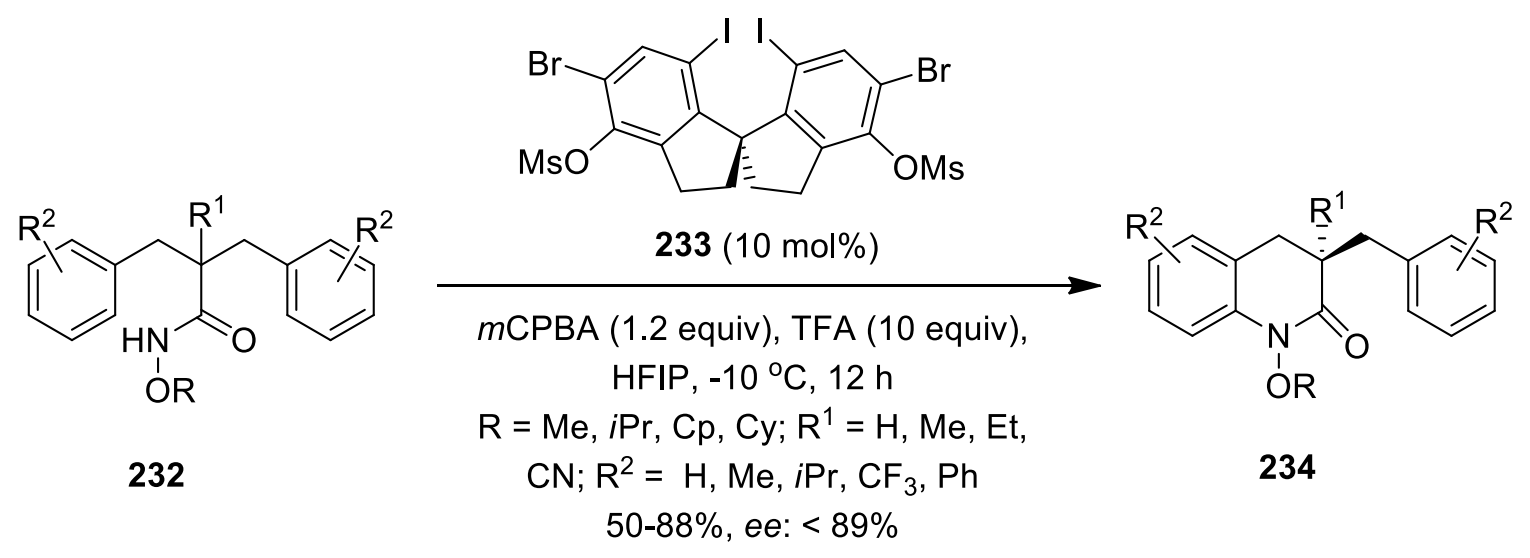

Scheme 62. Synthesis of $N$-alkoxy-lactams 234 using 233 as precatalyst. 
Wang and co-workers employed hypervalent iodine(III) reagent 236 as an efficient oxidant for the intramolecular decarboxylative Heck-type reaction of readily accessible 2-vinyl-phenyl oxamic acids 235 (Scheme 63). ${ }^{96}$ This operationally simple lactamization method enabled preparation of various 2-quinolinones 237 in variable yields with excellent chemoselectivity.<smiles></smiles>

235

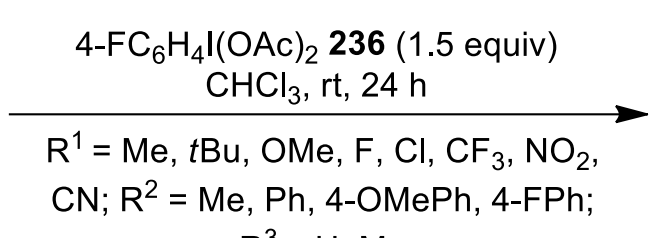

$\mathrm{R}^{3}=\mathrm{H}, \mathrm{Me}$

$52-96 \%$<smiles>[R]c1c([R])c2[c-][R1]ccc2[nH]c1=O</smiles>

237

Scheme 63. Synthesis of 2-quinolinones 237 using 4- $\mathrm{FC}_{6} \mathrm{H}_{4} \mathrm{I}(\mathrm{OAc})_{2} 236$ as the oxidant.

A plausible mechanism is elucidated in scheme 64. Initially, substrates $\mathbf{2 3 5}$ reacts with hypervalent iodine(III) reagent $\mathbf{2 3 6}$ giving cyclic iodine(III) monomer $\mathbf{2 3 8}$ which subsequently self-assembles to form macrocyclic trimer 239. The diradical intermediate $\mathbf{2 4 0}$ generated through ring-strain-induced homolysis of iodine-oxygen bond, undergoes decarboxylation and radical addition to the alkene to give intermediate $\mathbf{2 4 1}$. Next, intermediate $\mathbf{2 4 1}$ upon intramolecular aryliodine radical-mediated oxidation gives benzylic cation intermediate 242 with loss of $\operatorname{Arl}\left(4-\mathrm{FC}_{6} \mathrm{H}_{4} \mathrm{l}\right)$. Finally, E1 elimination of $\mathbf{2 4 2}$ delivers desired product $\mathbf{2 3 7} .^{96}$<smiles>[R]C=C([R])c1ccccc1NC(=O)C(=O)O</smiles>

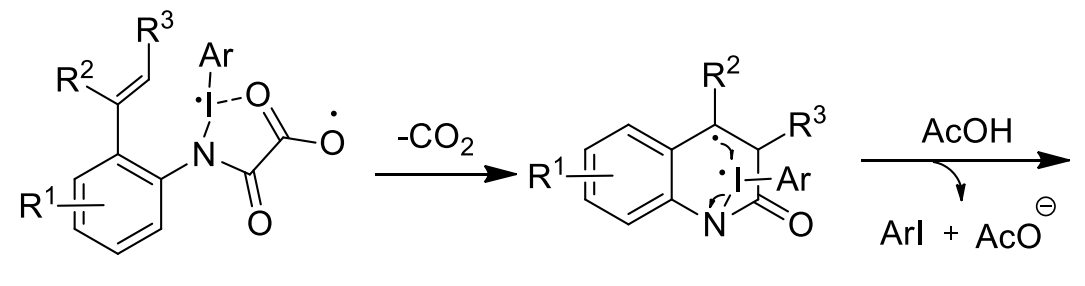

240

241

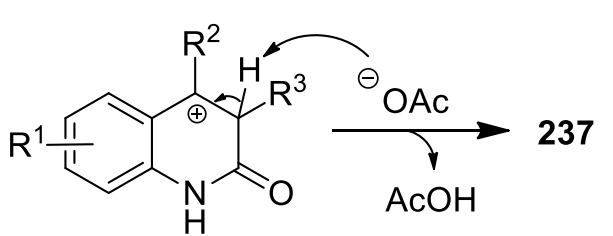

242

Scheme 64. Plausible mechanism of synthesis of 2-quinolinones 237 using 4- $\mathrm{FC}_{6} \mathrm{H}_{4}(\mathrm{OAC})_{2} 236$ as the oxidant.

Jacobsen and coworkers developed a catalytic route to prepare 4-fluoroisochromanones 245 through enantioselective fluorolactonization of vinyl benzoates $\mathbf{2 4 3}$ using chiral aryl iodide $\mathbf{2 4 4}$ as precatalyst (Scheme 65). ${ }^{97}$ Reaction employs HF-pyridine as a nucleophilic fluorinating reagents and $m$ CPBA as the terminal oxidant. This nucleophilic fluorination protocol enabled introduction of fluorine-containing stereogenic center, which 
constitute a frontier endeavor in organic synthesis. Moreover, reaction products are formed in the syn configuration as determined by X-ray crystallographic analysis. Reaction possibly occurs through intermediate 246, wherein anchimeric assistance of carboxylate group lead to the displacement of aryliodo group giving desired products.

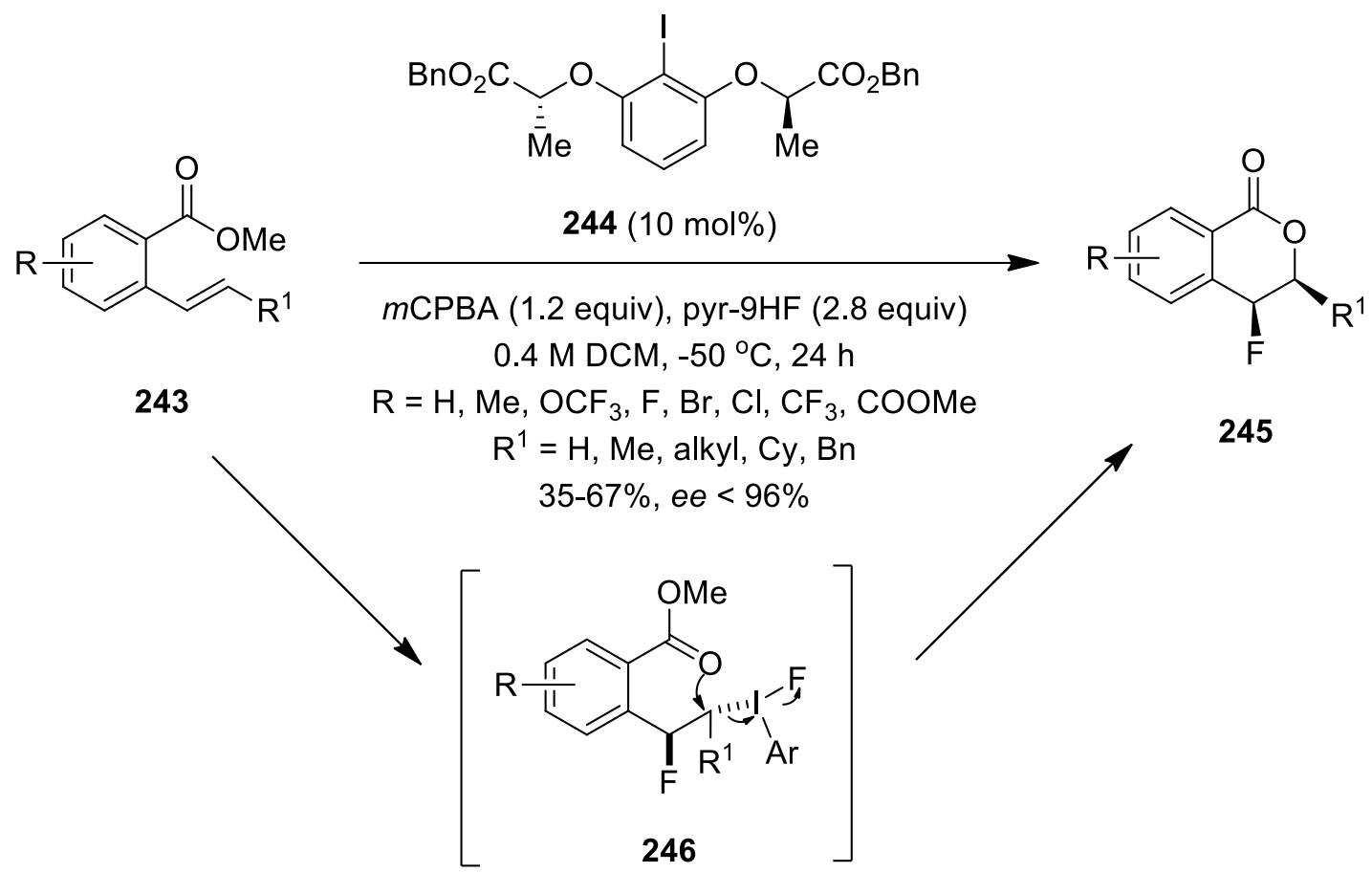

Scheme 65. Enantioselective synthesis of 4-fluoroisochromanones $\mathbf{2 4 5}$ using chiral aryl iodide $\mathbf{2 4 4}$ as catalyst.

Later, Möckel et al. developed a novel electrochemical method for the lactonization of vinyl benzoates 243 using as precatalyst iodobenzene 13 . The reaction was performed in the presence of lithium perchlorate and trifluoroacetic acid as electrolyte and supporting acid respectively. Trifluoroethoxy-substituted isochromanones 247 were isolated in appreciable yields (Scheme 66). ${ }^{98}$ Reaction scope was administered by changing the steric and electronic components of the substrates. Further functional group tolerance was determined using compatibility test and it indicated that functional groups labile to oxidative conditions show low yields. In case of vinyl substituted substrates, satisfying diastereomeric ratio were observed.

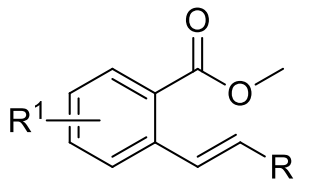

243

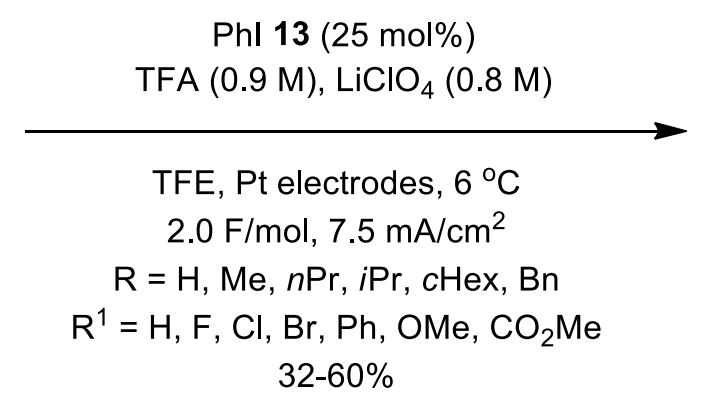

Phl 13 (25 mol\%)

F, Pt electrodes, $6^{\circ} \mathrm{C}$ 2.0 F/mol, $7.5 \mathrm{~mA} / \mathrm{cm}^{2}$ $\mathrm{R}=\mathrm{H}, \mathrm{Me}, \mathrm{nPr}, \mathrm{PPr}, \mathrm{cHex}, \mathrm{Bn}$ $32-60 \%$<smiles>[R1]c1ccc2c(c1)[C@H](OCC(F)(F)F)[C@@H]([R])OC2=O</smiles>

247

Scheme 66. Synthesis of isochromanones $\mathbf{2 4 7}$ using iododobenzene $\mathbf{1 3}$ as precatalyst. 
Cui's research group developed a metal-free route to prepare 2-hydroxy-benzo[b][1,4]oxazins 249 from $N$ (2-hydroxylaryl)enaminones 248 using PIDA 6 under air atmosphere (Scheme 67). ${ }^{99}$ This one-pot synthesis exhibits excellent functional group compatibility with broad substrates scope and significant product yields. The proposed mechanism initiates with the 1,5- $\mathrm{H}$ shift of $\mathbf{2 4 8}$ to give iminoenolate intermediate 250, followed by PIDA-induced oxidation to provide spirolactone intermediate 251 which reversibly forms $\mathbf{2 5 2}$. Further $\mathrm{Et}_{3} \mathrm{~N}$ promoted oxidation of $\mathbf{2 5 2}$ under $\mathrm{O}_{2}$ gives superoxide radical intermediate $\mathbf{2 5 3}$ which upon subsequent dismutation generates intermediate 254 and releases hydroxyl radical. This radical could be then trapped by $\mathbf{2 5 2}$ to continue the radical chain growth in the presence of $\mathrm{O}_{2}$. Finally, intramolecular cyclization of 254 furnishes desired product 249.

EtOH, rt, air

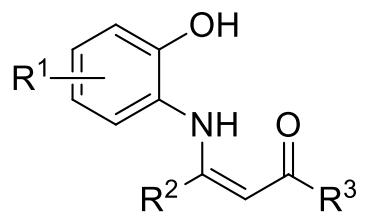

1) PIDA 6 (1.2 equiv), $10 \mathrm{~min}$

2) $\mathrm{Et}_{3} \mathrm{~N}$ (2.0 equiv). $\mathrm{R}^{3}=\mathrm{Ar}, 2$-furyl, 2-thienyl, 2-naphthyl

$37-86 \%$<smiles>[R]CC1(O)OC2=C(C=C[R1]([R])C=C2)N=C1[R]</smiles>

249

248

1,5-H shift<smiles>[R]C(O)=CC([R])=Nc1ccccc1O</smiles>

250<smiles>[R]C1=CC=CC2(N=C([R])C=CC2=O)O1</smiles>

251

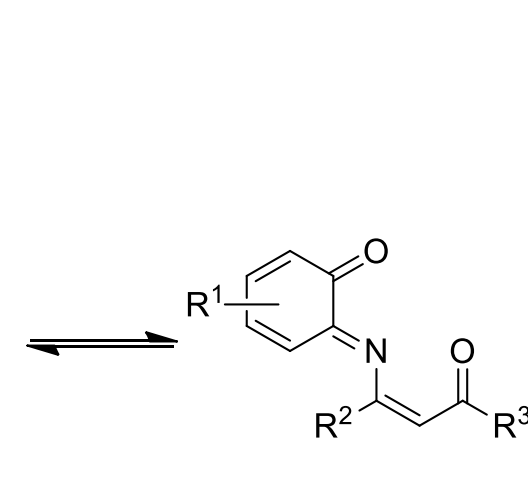

252

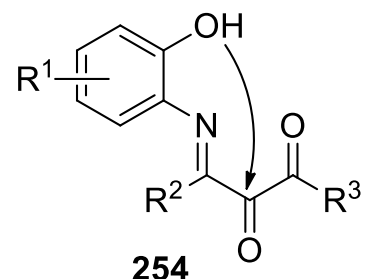

254

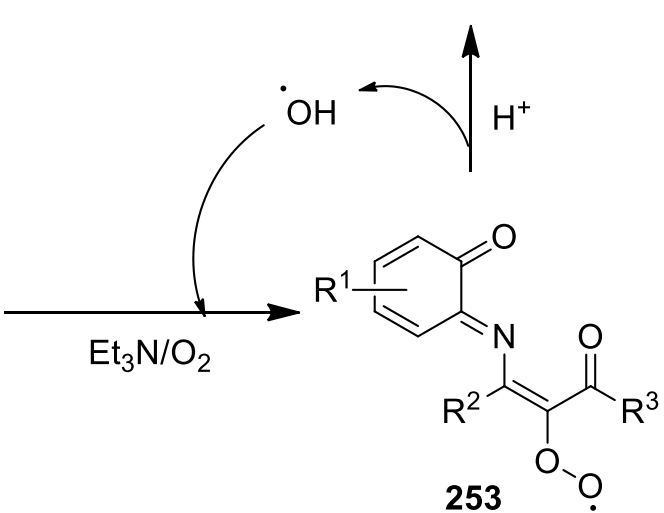

Scheme 67. Preparation of 2-hydroxy-benzo[b][1,4]oxazines 249 using PIDA 6 as an oxidant.

In 2016, Wengryniuk's research group reported synthesis of benzo-fused oxygen heterocycles 257 via oxidative rearrangement of benzylic tertiary alcohols 255. This reaction was facilitated by (poly)cationic hypervalent iodine reagent $\mathbf{2 5 6}$ promoting C-to-O alkyl migration and represents the first example showing the unique reactivity of this class of reagents (Scheme 68). ${ }^{100}$ Although detailed mechanism is not provided, authors envisioned attack of the alcohol on the iodine center that would generate an activated intermediate 258 followed by carbon to oxygen alkyl migration to generate oxonium ion 259 which could be trapped by a 
nucleophile to give cyclic ethers 257. Reaction was highly scalable, demonstrated by gram scale reaction and also HFIP-derived acetals $\mathbf{2 5 7}$ were subjected to subsequent derivatization under different reaction conditions.

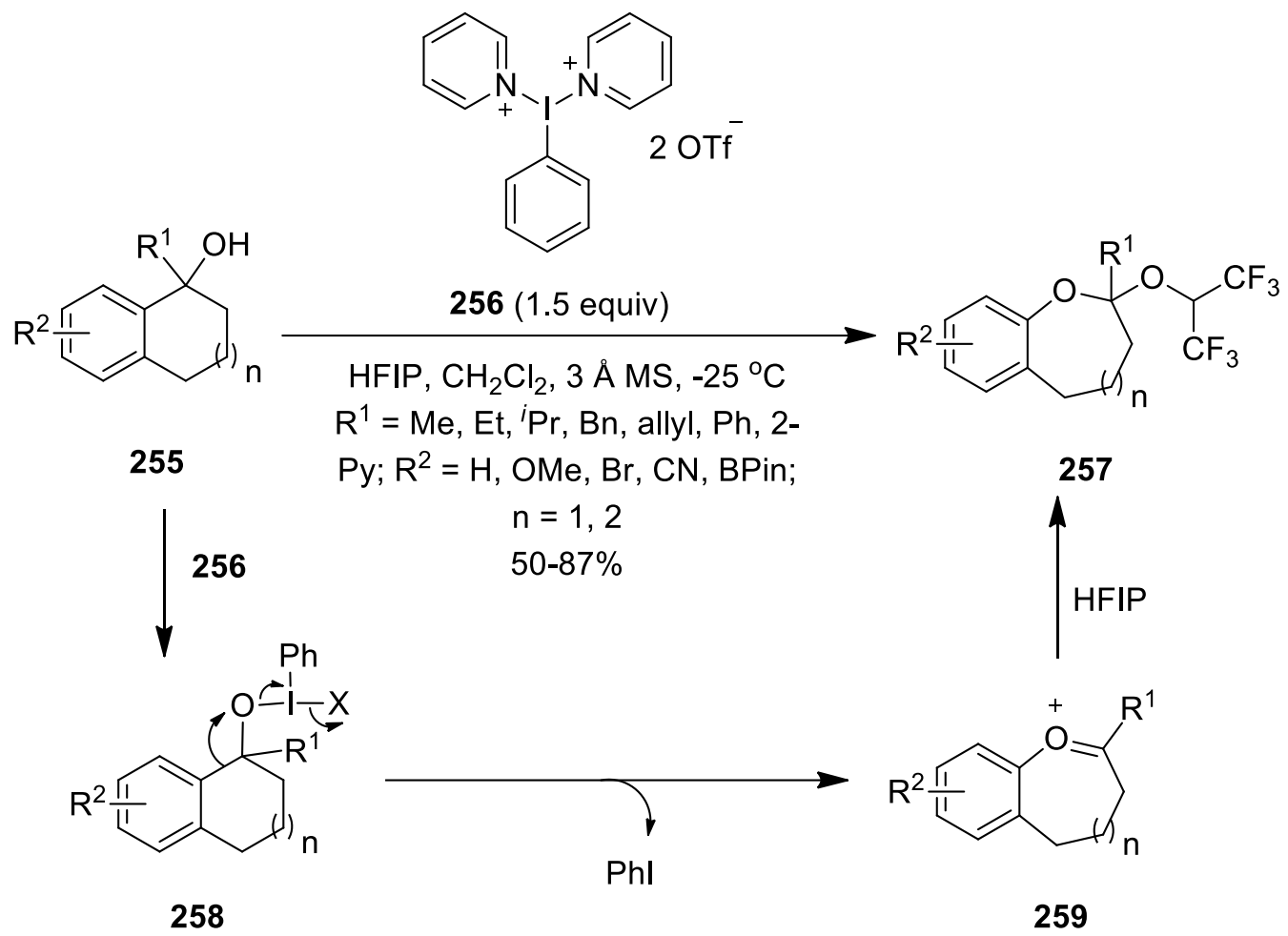

Scheme 68. Synthesis of Benzo-fused oxygen heterocycles 257 using polycationic hypervalent iodine reagent 256.

In 2019, PIDA-induced oxidative rearrangement of primary amines 260 via 1,2-C to $\mathrm{N}$ migration was developed by Murai's research group. This method enabled facile synthesis of cyclic amines such as benzoazepine $261(n=1)$ and benzosuberan $261(n=2)$ in significant yields (Scheme 69). ${ }^{101}$ Substituents such as chloro, methoxy, ester and trifluoromethyl groups were well tolerated.<smiles>[R]c1ccc2c(c1)[14CH2]CC2(N)N</smiles>

260
1) PIDA 6 (1.3 equiv), $\mathrm{Cs}_{2} \mathrm{CO}_{3}$ (2.6 equiv),

$$
\begin{aligned}
& \text { 2) } \mathrm{NaBH}_{4} \text { (5.0 equiv), } 0^{\circ} \mathrm{C} \text { to rt, } 1-7 \mathrm{~h} \\
& \mathrm{R}=\mathrm{H}, \mathrm{Cl}, \mathrm{OMe}, \mathrm{CO}_{2} \mathrm{Me} \text {, } \\
& \mathrm{CF}_{3} ; \mathrm{n}=1,2 \\
& 40-95 \%
\end{aligned}
$$<smiles>[R]c1ccc2c(c1)NCCC(C)N2</smiles>

261

Scheme 69. Synthesis of cyclic amines 261 using PIDA 6 as an oxidant.

\subsection{Synthesis of polycyclic heterocycles}

Maiti and Mal designed a PIDA-induced intermolecular dehydrogenative annulation strategy for the synthesis of carbazoles 264 from non-prefunctionalized $N$-sulfonylanilides 262 and 1,3,5-trialkylbenzenes 263 (Scheme 70). ${ }^{102}$ This tandem $\mathrm{C}-\mathrm{C} / \mathrm{C}-\mathrm{N}$ bond forming reaction involves simultaneous functionalization of three $\mathrm{C}\left(s p^{2}\right)-\mathrm{H}$ 
and one $\mathrm{N}\left(s p^{3}\right)-\mathrm{H}$ bonds followed by one alkyl migration. Further scope of this annulation method was extended with substrates 262 containing $-\mathrm{H}$ at the para-position $\left(\mathrm{R}^{3}=\mathrm{H}\right)$, enabling synthesis of multi-substituted carbazols 265 via sequential five $\mathrm{C}-\mathrm{H}$ and one $\mathrm{N}-\mathrm{H}$ bond functionalization.

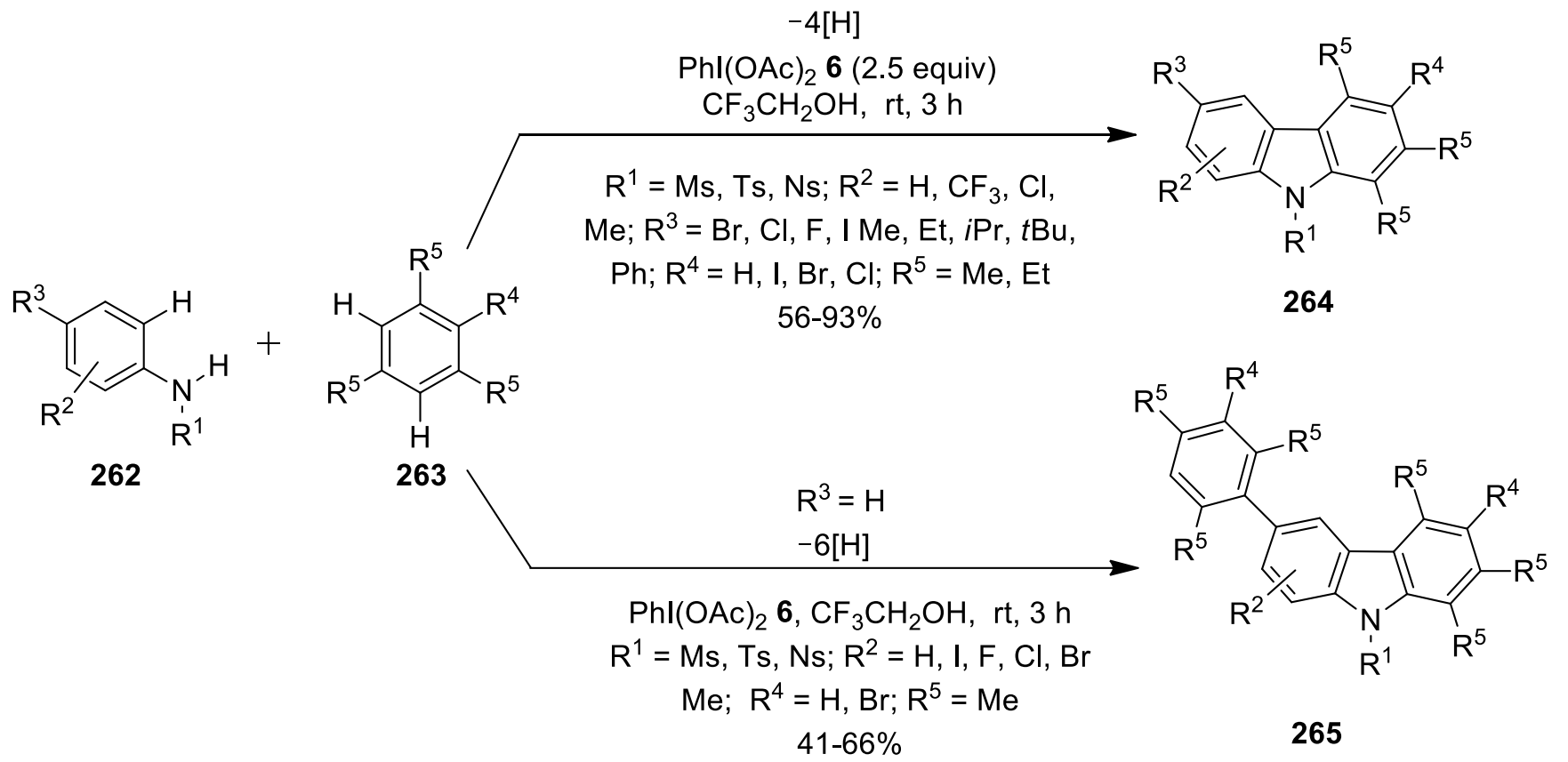

Scheme 70. Synthesis of multi-substituted carbazoles 264 and 265 using oxidant PIDA 6.

The proposed mechanism for this intermolecular reaction is depicted in scheme $71 .{ }^{102}$ Initially, anilide 262 interacts with PIDA 6 to form nitrenium ion intermediate $\mathbf{2 6 7}$ which later stabilizes through charge delocalization to give carbenium ion 268. C-arylated intermediate 269, obtained through nucleophilic addition of arene 263 to the 268, undergoes further oxidation with PIDA 6 to generate ionic intermediate $\mathbf{2 7 0}$. Subsequent electrophilic aromatic substitution furnishes carbenium intermediate $\mathbf{2 7 1}$ which is stabilized by neighbouring quaternary methyl group migration. Finally, conversion of cationic intermediate $\mathbf{2 7 2}$ into the heterocyclic product $\mathbf{2 6 4}$ occurs via abstraction of proton by acetate ion. 


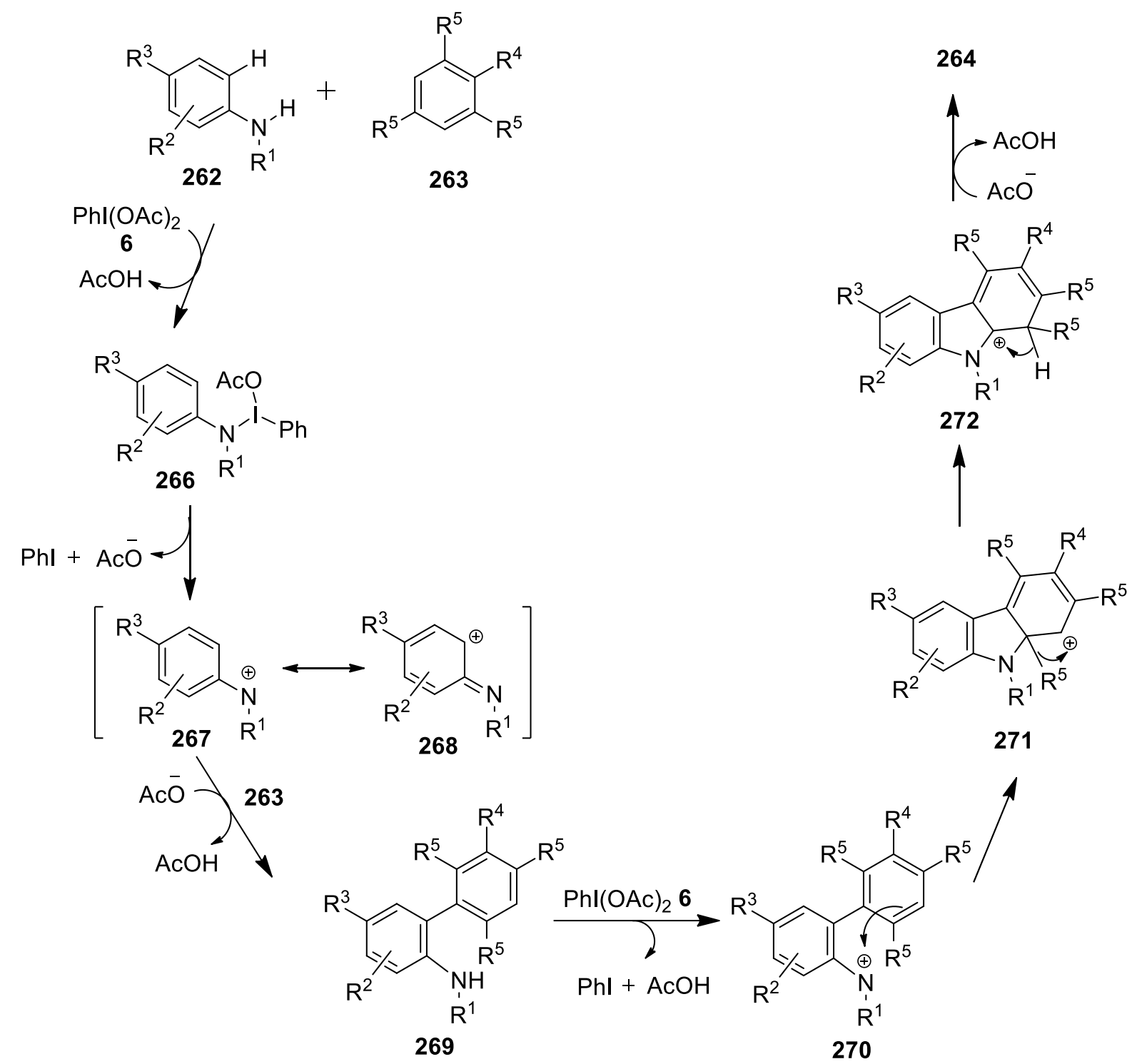

Scheme 71. Proposed mechanism for the synthesis of multi-substituted carbazoles 264.

In continuation, Mal's group employed iodine(III) reagent as sole oxidant to prepare multi-substituted carbazoles from anilides $\mathbf{2 6 2}$ and simple arenes $\mathbf{2 6 3}$ either by using stoichiometric PIDA $\mathbf{6}$ (Method A) or catalytic Phl-mCPBA system (Method B) (Scheme 72). ${ }^{103}$ Reactions were performed at ambient temperature and tolerates range of functional groups. Notably, stoichiometric pathway provided better yields as compared to catalytic ones. Further synthetic utility of this method was well documented in the synthesis of bio-active natural products. 


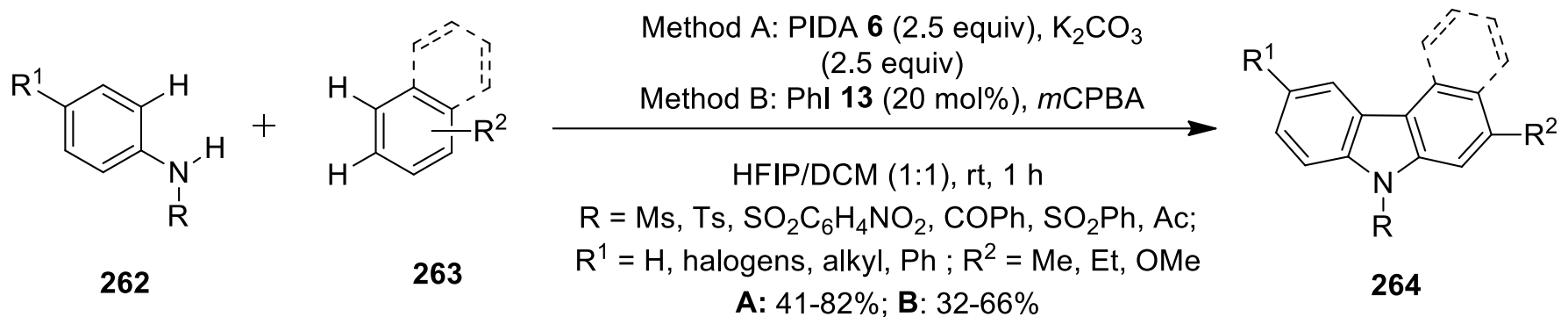

Scheme 72. Hypervalent iodine(III)-mediated synthesis of multi-substituted carbazoles 264.

Later, the same group developed intramolecular dehydrogenative $\mathrm{C}-\mathrm{N}$ coupling reaction for the synthesis of carbazoles 264 by reacting biarylsulfonanilides $\mathbf{2 7 3}$ with iodine(III) reagent (Scheme 73). ${ }^{104}$ This method enabled distal (-meta) $\mathrm{C}-\mathrm{H}$ bond functionalization with the aid of 1,2-alkyl migration. Reactions were performed either by using stoichiometric phenyliodine diacetate 6 or in situ generated iodine(III) reagent from precatalyst iodobenzene 13 (20 mol \%) and terminal oxidant $m$ CPBA. Substrates 273 with electron-rich arene moiety gave higher products yield as compared to electron-deficient ones. Both reaction pathways worked perfectly well at room temperature under open atmosphere condition. Further conversion of $N$-protected carbazole 264 into the corresponding $\mathrm{NH}$-carbazole derivative was done by treating with $\mathrm{Cs}_{2} \mathrm{CO}_{3}$ in THF-MeOH under refluxing condition.<smiles>[R]Nc1ccc([R])cc1-c1c([R])cc([R])c([R])c1[R]</smiles>

273
Method A: PIDA 6 (1.2 equiv), TFE, rt, $3 \mathrm{~h}$ Method B: Phl 13, $m$ CPBA, TFE, rt, $3 \mathrm{~h}$

$\mathrm{R}=\mathrm{Ms}, \mathrm{SO}_{2} \mathrm{Et}, \mathrm{SO}_{2} \mathrm{Ph}, \mathrm{Ts}, \mathrm{Ns} ; \mathrm{R}^{1}=$ $\mathrm{Br}, \mathrm{Cl}, \mathrm{I}, \mathrm{F}, \mathrm{Me}, \mathrm{Et}, i \mathrm{Pr}, t \mathrm{Bu} ; \mathrm{R}^{2}=\mathrm{Me}$, $\mathrm{Et} ; \mathrm{R}^{3}=\mathrm{H}, \mathrm{Br}$ A: $38-98 \%$; B: $35-97 \%$<smiles>[R]c1ccc2c(c1)-c1c([R])c([R])c([R])c(c1[R])N2[R]</smiles>

264

Scheme 73. Synthesis of carbazoles 264 using hypervalent iodine(III) reagent as an oxidant.

Murai and co-workers performed the first oxidative rearrangement of cyclic secondary amines $\mathbf{2 7 4}$ using hypervalent iodine reagent 6 (Scheme 74). ${ }^{105}$ This method comprises PhI(OAc) 2 -promoted 1,2-C-to-N alkyl migration of secondary amines $\mathbf{2 7 4}$ followed by subsequent reduction using $\mathrm{NaCNBH}_{3}$ to provide tetracyclic compounds 275. Further scope of the reaction was extended towards the synthesis of macrocyclic indole-fused compounds. 


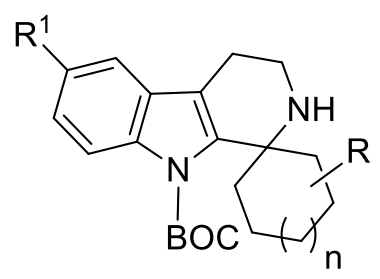

274

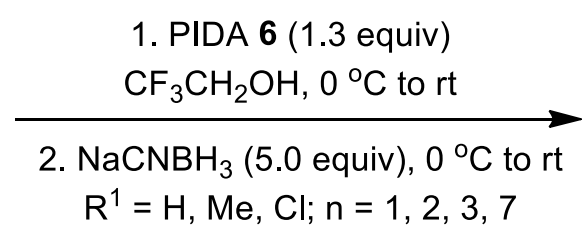
$52-89 \%$

$R=\left\{\begin{array}{l}r_{2}^{5} \\ 2\end{array}\right\}$

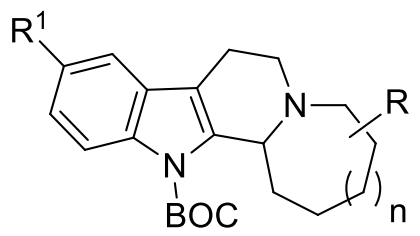

275

Scheme 74. Synthesis of tetracyclic indole-fused compounds $\mathbf{2 7 5}$ using PIDA 6 as an oxidant.

Meanwhile, Sugimura's team presented an enantioselective intramolecular oxyarylation of $(E)-6$-aryl-1-

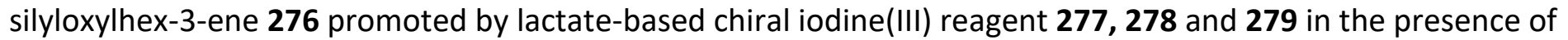
$\mathrm{BF}_{3} \cdot \mathrm{OEt}_{2}$ (Scheme 75). ${ }^{106}$ Tricyclic products $\mathbf{2 8 0}$ were obtained in variable yields under metal-free conditions. Further experimental evidences revealed that silyl group as a protecting group accelerates this oxidative cyclization reaction and also contribute for high enantioselectivity. Additionally, aminoarylation of methanesulfonylamide provided hexahydrobenz[e]indole in $85 \%$ yield (ee $80 \%$ ) using tris(pentafluorophenyl)borane as promoter.

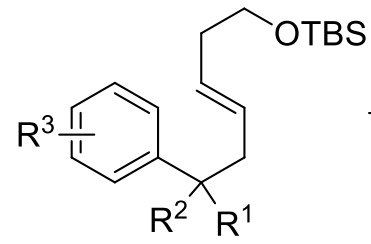

276<smiles></smiles>

277

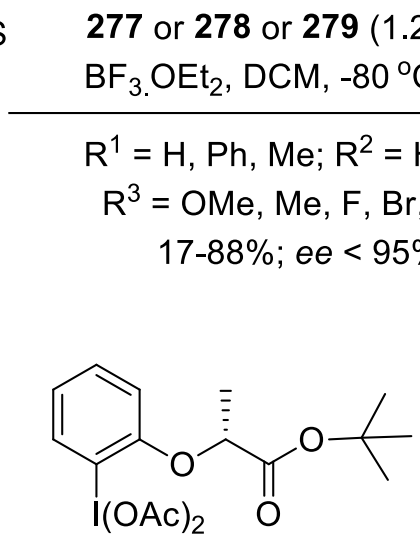

278

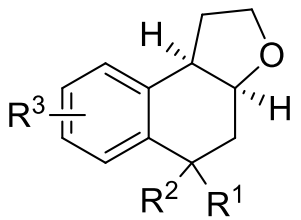

280<smiles>COC(=O)[C@H](C)Oc1cccc(O[C@H](C)C(=O)OC)c1OC</smiles>

279

Scheme 75. Enantioselective synthesis of tricyclic products $\mathbf{2 8 0}$ using lactate-based chiral iodine(III) reagent.

In 2016, Waghmode et al. employed PIDA 6 as an oxidant to prepare 1,3-napthoxazines 282 through cross dehydrogenative-coupling of 1-( $\alpha$-aminoalkyl)-2-naphthols 281 (Scheme 76). ${ }^{107}$ The precursors 281 were synthesized via three-component condensation of $\beta$-naphthol, aldehydes and cyclic secondary amines via Betti reaction. The proposed mechanism initiates with the reaction of $\mathbf{2 8 1}$ with PIDA $\mathbf{6}$ to form intermediate $\mathbf{2 8 3}$ via ligand exchange, which further gives six membered iodine(III) heterocycle $\mathbf{2 8 4}$. Intermediate $\mathbf{2 8 4}$ upon reductive elimination of Phl generates imminium ion $\mathbf{2 8 5}$ followed by subsequent trapping by phenoxide anion to yield product 282. 


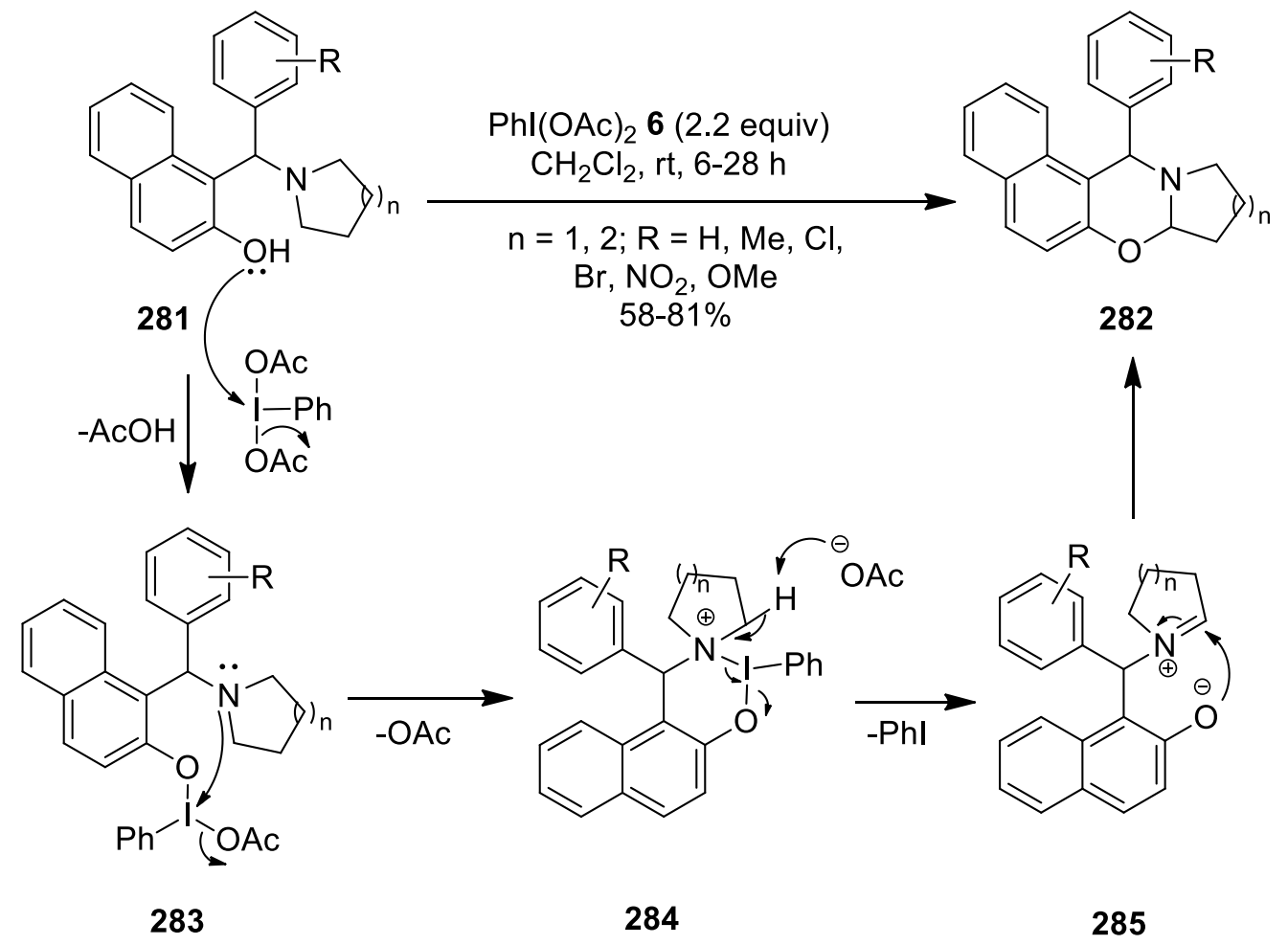

Scheme 76. Synthesis of 1,3-napthoxazines 282 using as oxidant PIDA 6.

In 2017, Hong et al. established an efficient protocol for the one-pot synthesis of $7 H$-chromeno[3,2c]quinolones $\mathbf{2 8 8}$ from arylols $\mathbf{2 8 7}$ and substituted aryliodine(III) reagents $\mathbf{2 8 6}$ through cascade $\mathbf{O}$-arylation and palladium-catalyzed $\mathrm{C}\left(\mathrm{sp}^{3}\right)-\mathrm{H}$ arylation process (Scheme 77). ${ }^{108}$ Both electron-donating and -withdrawing substituents on the quinoline ring were well tolerated. Further 4-hydroxycoumarin $\mathbf{2 8 9}$ was converted into benzopyranone derivative $\mathbf{2 9 0}$ using bis(acetoxy)iodoarene $\mathbf{2 8 6}$ under similar conditions in 59\% yield. 


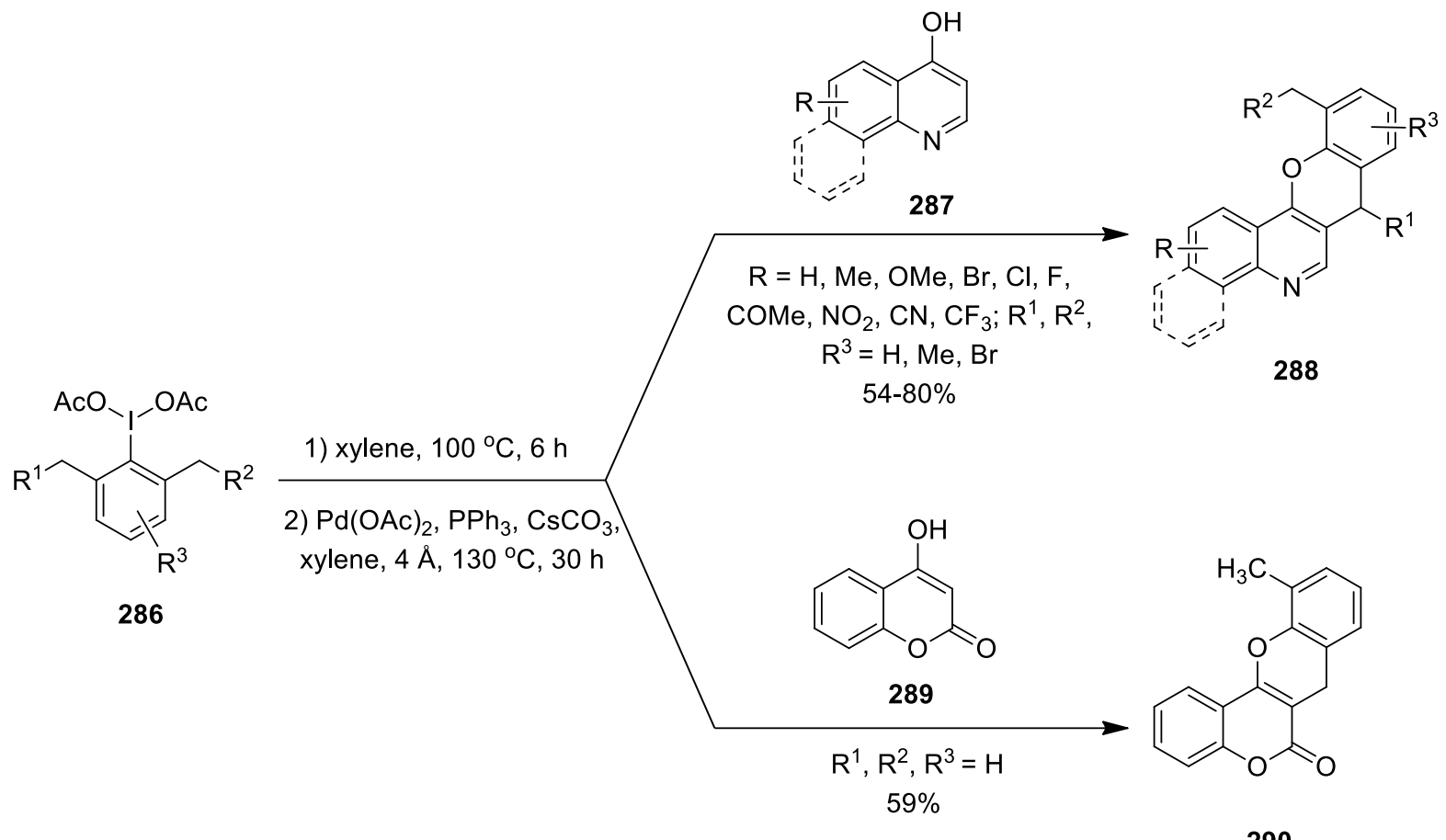

Scheme 77. Synthesis of $7 H$-chromeno[3,2-c]quinolones $\mathbf{2 8 8}$ and benzopyranone derivative $\mathbf{2 9 0}$ using trivalent aryliodine reagents 286 .

In 2019, Chen et al. disclosed an interesting $\mathrm{N}$-heterocyclic carbene (NHC) 292-catalyzed intramolecular domino reaction of aryl aldehyde 291 using PIDA 6 (Scheme 78). ${ }^{109}$ Based on the control experiments and DFT studies, a domino two-stage mechanism was proposed involving NHC-catalyzed oxidation of the aldehyde to the corresponding carboxylic acid via acyl azolium intermediate formed from Breslow intermediate 293 and subsequent addition of carboxylate to the iminium intermediate to give desired product 294. Several cyclic amines such as piperidine, pyrrolidine, morpholine and azepine-derived aldehydes were well reacted under optimized conditions. 


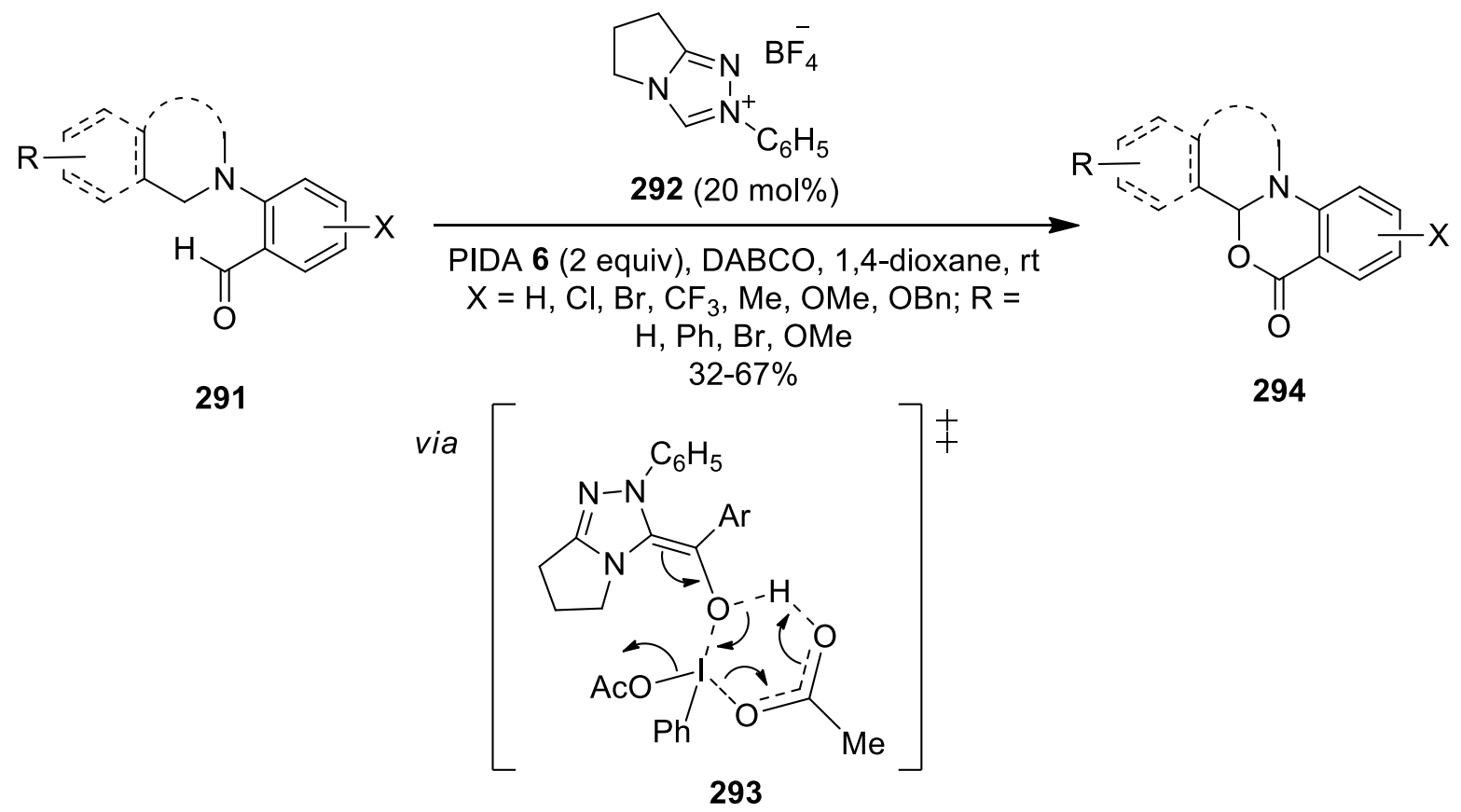

Scheme 78. Synthesis of $\alpha$-oxygenated products 294 using PIDA 6 as an oxidant.

Deng et al. established a new strategy to construct polycyclic cyclohexadienones 298 through intramolecular alkoxy-oxylactonization/dearomatization of 3'-hydroxy-[1,1'-biphenyl]-2-carboxylic acid 295 promoted by stoichiometric oxidant PIDA 6 (Scheme 79). ${ }^{110}$ Further asymmetric version of this method was developed by using in situ generated aryl- $\lambda^{3}$-iodane from chiral aryl iodide $\mathbf{2 9 6}$ in the presence of $m$-CPBA in MeOH. Reaction scope was investigated by employing different alcohols 297 as nucleophile. Notably, decrease in product yields and enantioselectivities was observed for sterically hindered alcohols and therefore reaction was found sensitive to the size of the alcohol. Further methyl-substituted substrate $\mathbf{2 9 5}(R=\mathrm{Me})$ enabled synthesis of core unit of dehydroaltenusin $\mathbf{2 9 9}$, which is an inhibitor of DNA polymerase.

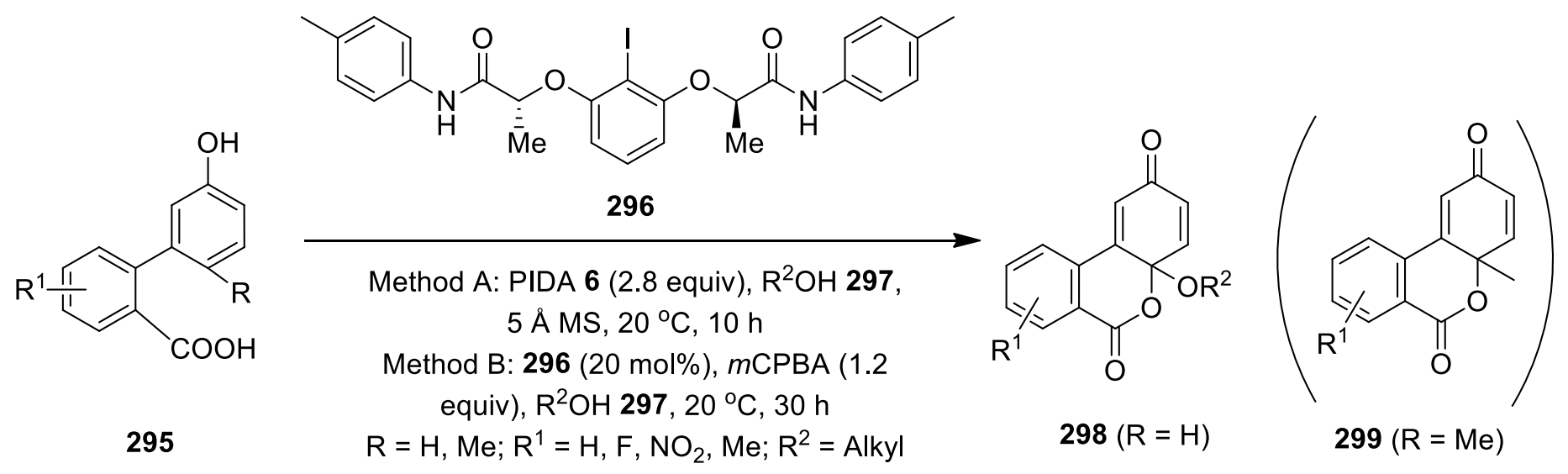

A: $48-80 \%$; B: $21-61 \%$, ee: $<52 \%$

Scheme 79. Hypervalent iodine(III)-mediated synthesis of polycyclic cyclohexadienones 298. 


\subsection{Synthesis of spirocyclic heterocycles}

Oxidative dearomatizative spirocyclization constitutes an important platform for the preparation of functionalized spirocyclic skeletons. Chiral hypervalent iodine reagents are frequently employed as reagents or catalyst to achieve asymmetric dearomatization of phenols and other related electron-rich organic compounds. The current section of the review highlights the recent progress made in the enantioselective dearomatizative spirocyclization reactions. In 2015, Zhang et al. constructed spirooxindole derivatives 301 from 1-hydroxy- $N$ aryl-2-naphthamides $\mathbf{3 0 0}$ via chiral organoiodine-catalyzed enantioselective oxidative dearomatization process (Scheme 80). ${ }^{111}$ This reaction enabled stereoselective creation of all-carbon stereogenic center containing spiro products 301 in good yields with excellent enantioselectivities (up to 92\% ee). Notably, the active hypervalent species, phenyl- $\lambda^{3}$-iodanes generated in situ through mCPBA-mediated oxidation of chiral iodoarene 102 catalyze this asymmetric spirocyclization reaction.

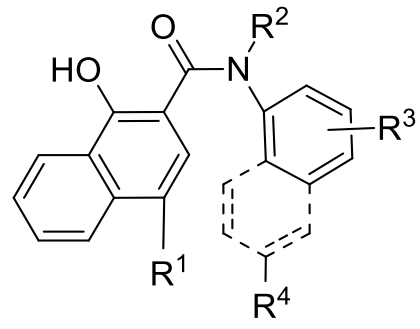

300

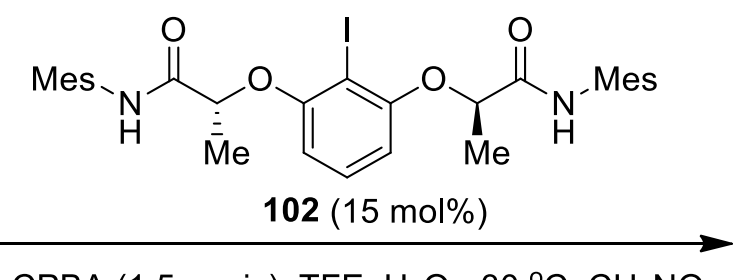

mCPBA (1.5 equiv), TFE, $\mathrm{H}_{2} \mathrm{O},-30^{\circ} \mathrm{C}, \mathrm{CH}_{3} \mathrm{NO}_{2}$ $\mathrm{R}^{1}=\mathrm{H}, \mathrm{Br} ; \mathrm{R}^{2}=\mathrm{Me}, \mathrm{Et}, \mathrm{Ph} ; \mathrm{R}^{3}=\mathrm{H}, \mathrm{Me}, \mathrm{OMe}$, $t \mathrm{Bu}, \mathrm{Me} ; \mathrm{R}^{4}=\mathrm{H}, \mathrm{Me}, \mathrm{OMe}, \mathrm{OBn}$ 42-80\%; ee: $<92 \%$

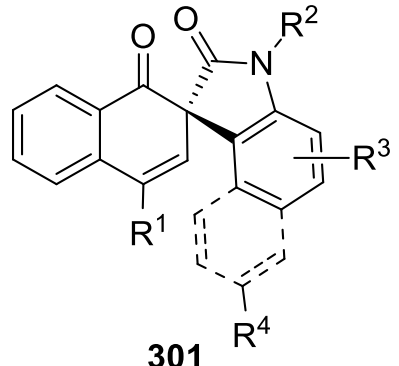

301

Scheme 80. Synthesis of spirooxindoles 301 using chiral iodoarene 102 as precatalyst.

Later, Murphy's research team presented an unprecedented metal-free approach to access 3,3'spirooxindolo dihydrofurans 303 by reacting cyclic iodonium ylides 183 with 3-alkylidene-2-oxindoles 302 using $\mathrm{Bu}_{4} \mathrm{NI}$ catalysis (Scheme 81). ${ }^{112}$ The reaction was tolerant to a variety of electron-poor and electron-neutral substituents on the alkylidene substrates and the products were isolated in high to excellent yields. Other iodonium ylides derived from 1,3-diketones, pyrimidines and 1,3-ketoesters smoothly gave spirocyclic products in significant yields.

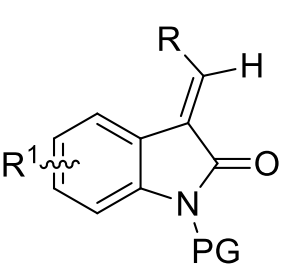

302

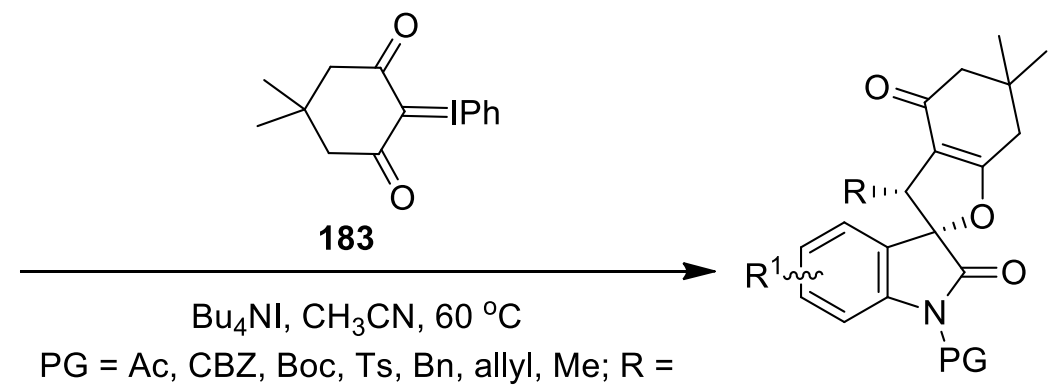
$\mathrm{CO}_{2} \mathrm{Et}, \mathrm{COPh}, \mathrm{COMe}, \mathrm{Ph} ; \mathrm{R}^{1}=\mathrm{H}, \mathrm{F}, \mathrm{Br}, \mathrm{Cl}$

$73-99 \%$
303

Scheme 81. Synthesis of spirooxindolo dihydrofurans 303 by reacting cyclic iodonium ylides 183 with 3-alkylidene-2-oxindoles 302. 
In 2017, Ishiara's group adapted oxidative dearomatization strategy to prepare enantioselective masked ortho-benzoquinones $\mathbf{3 0 6}$ and $\mathbf{3 0 8}$ from ortho-hydroquinone derivatives using chiral organoiodine(III) catalysis (Scheme 82). ${ }^{113}$ Reactions works well with both phenols $O$-tethered to an acetic acid $\mathbf{3 0 4}$ or to an ethanol unit $\mathbf{3 0 7}$ by employing chiral iodoarene $\mathbf{3 0 5}$ as precatalyst. Further the use of synthesized spiroketal in the asymmetric synthesis of natural product, bis(monoterpene) (-)-biscarvacrol highlights the potential scope of this method. Additionally, synthesis of dioxolanone-type masked para-benzoquinones from para-hydroquinone derivatives were achieved under similar conditions with ee up to $89 \%$.<smiles>[R]c1ccccc1OCC(=O)O</smiles>

304<smiles>CC(=O)NCC(C)Oc1cccc(OC(C)C)c1I</smiles>

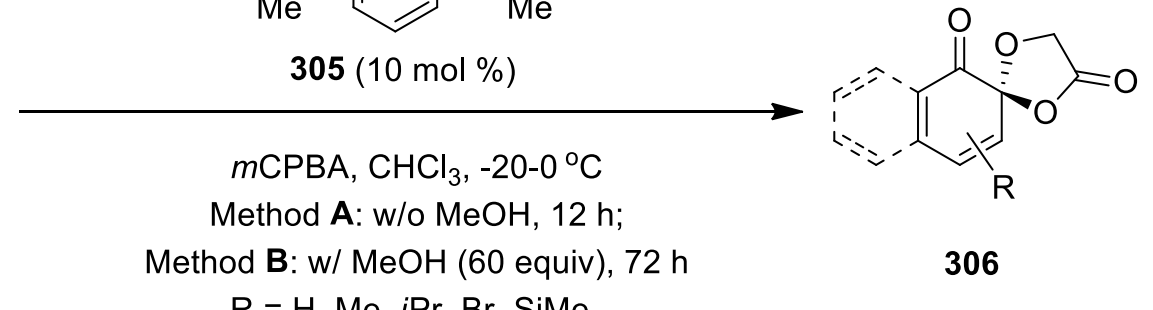

$$
\mathrm{R}=\mathrm{H}, \mathrm{Me}, \mathrm{PPr}, \mathrm{Br}, \mathrm{SiMe}_{3}
$$

A: $78-99 \%$, ee: $<84 \%$; B: $75-95 \%$, ee: $<96 \%$

$$
305 \text { (10 mol\%), } m \mathrm{CPBA}, 0^{\circ} \mathrm{C}, 11-24 \mathrm{~h}
$$

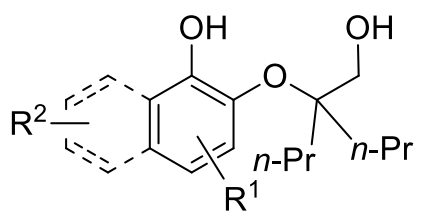

307
Method C: Pre-sonicated toluene/ $\mathrm{H}_{2} \mathrm{O}(5 / 1, \mathrm{v} / \mathrm{v})$

Method D: Pre-sonicated $\mathrm{CHCl}_{3} / \mathrm{H}_{2} \mathrm{O}(5 / 1$, v/v)

$\mathrm{R}^{1}=\mathrm{H}, \mathrm{Cl}, \mathrm{Ph}, \mathrm{Me}, \mathrm{OMe}, 4-i \mathrm{Pr}-6-\mathrm{SiMe}_{3}$,

3,5,6- $\mathrm{Me}_{3}-4-\mathrm{Cl}, 4-\mathrm{Br}, 5-6-\left(\mathrm{CH}_{2}\right)_{4}$

C: $53-81 \%$, ee: $<93 \%$; D: $54-80 \%$, ee: $<91 \%$

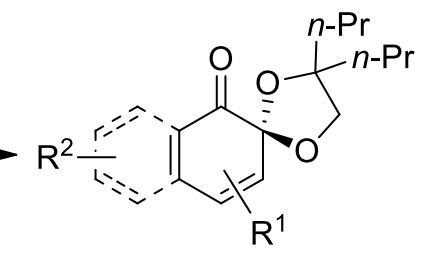

308

Scheme 82. Synthesis of masked ortho-benzoquinones $\mathbf{3 0 6}$ and $\mathbf{3 0 8}$ using chiral organoiodine(III) catalyst $\mathbf{3 0 5 .}$

In continuation, the same team employed organoiodine catalyst $\mathbf{3 0 5}$ for the enantioselective intramolecular oxidative dearomatization of naphthol derivatives 309 using mCPBA as an oxidant (Scheme 83). ${ }^{114}$ This conformationally flexible catalyst $\mathbf{3 0 5}$ was found very effective for inducing excellent enantioselectivities to the corresponding spirolactones $\mathbf{3 1 0}$ (ee up to 98\%). Notably, presence of HFIP and ethanol as an additive for the oxidation of 2-naphthols and 1-naphthols respectively was necessary for achieving high enantioselectivity.

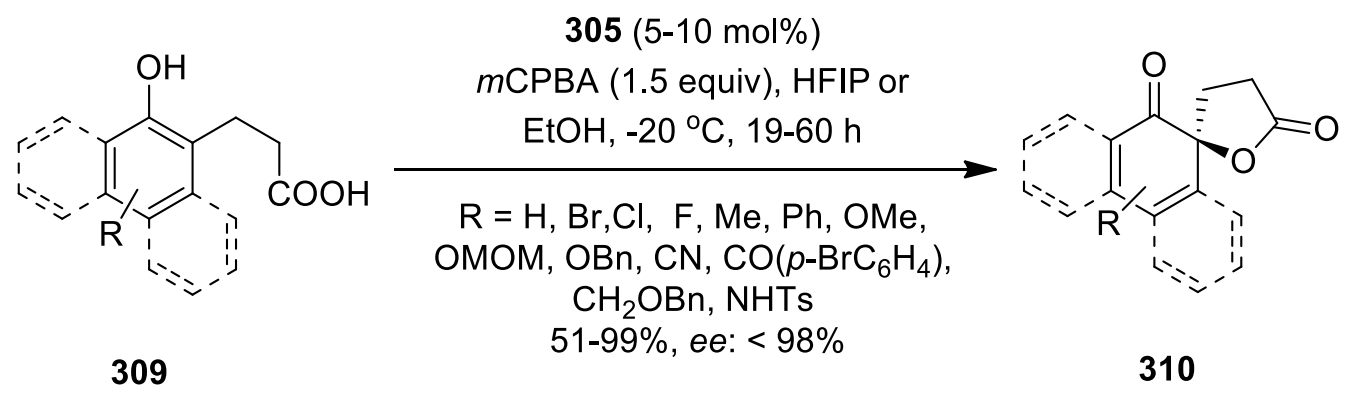

Scheme 83. Synthesis of spirolactones $\mathbf{3 1 0}$ using organoiodine catalyst 305. 
In the same year, Nachtsheim and co-workers designed a new C1 symmetric triazole-based chiral iodoarene catalyst 311 and successfully utilized this compound for the intramolecular asymmetric Kita-type spirolactonization of 4-substituted 1-naphthols 309. ${ }^{115}$ This method provided spirolactones 312 in variable yields and high enantioselectivity, facilitated by in situ generated hypervalent iodine(III) species using terminal oxidant $m C P B A$ (Scheme 84). Reaction scope was investigated under distinct conditions that is by maintaining reaction temperature to $0^{\circ} \mathrm{C}$ (Method $\mathrm{A}$ ) and $-20^{\circ} \mathrm{C}$ (Method B), and by using catalytic amount of $\mathbf{3 1 1}$ (Method C). Though this "first-generation" triazole-based catalyst provided highest enantioselectivities for this reaction compared to other C1-symmetric iodoarenes, their reactivities were comparatively low. Therefore, the same group synthesized "second-generation" triazole-based catalyst $\mathbf{3 1 3}$ by introducing ortho-substituent at the aryl iodide. ${ }^{116}$ This catalyst showed remarkable reactivity and excellent selectivity in the oxidative spirocyclization of 309. The spirolactone $\mathbf{3 1 0}$ was obtained in $85 \%$ yield with $99 \%$ ee, the highest enantioselectivities observed for this reaction.
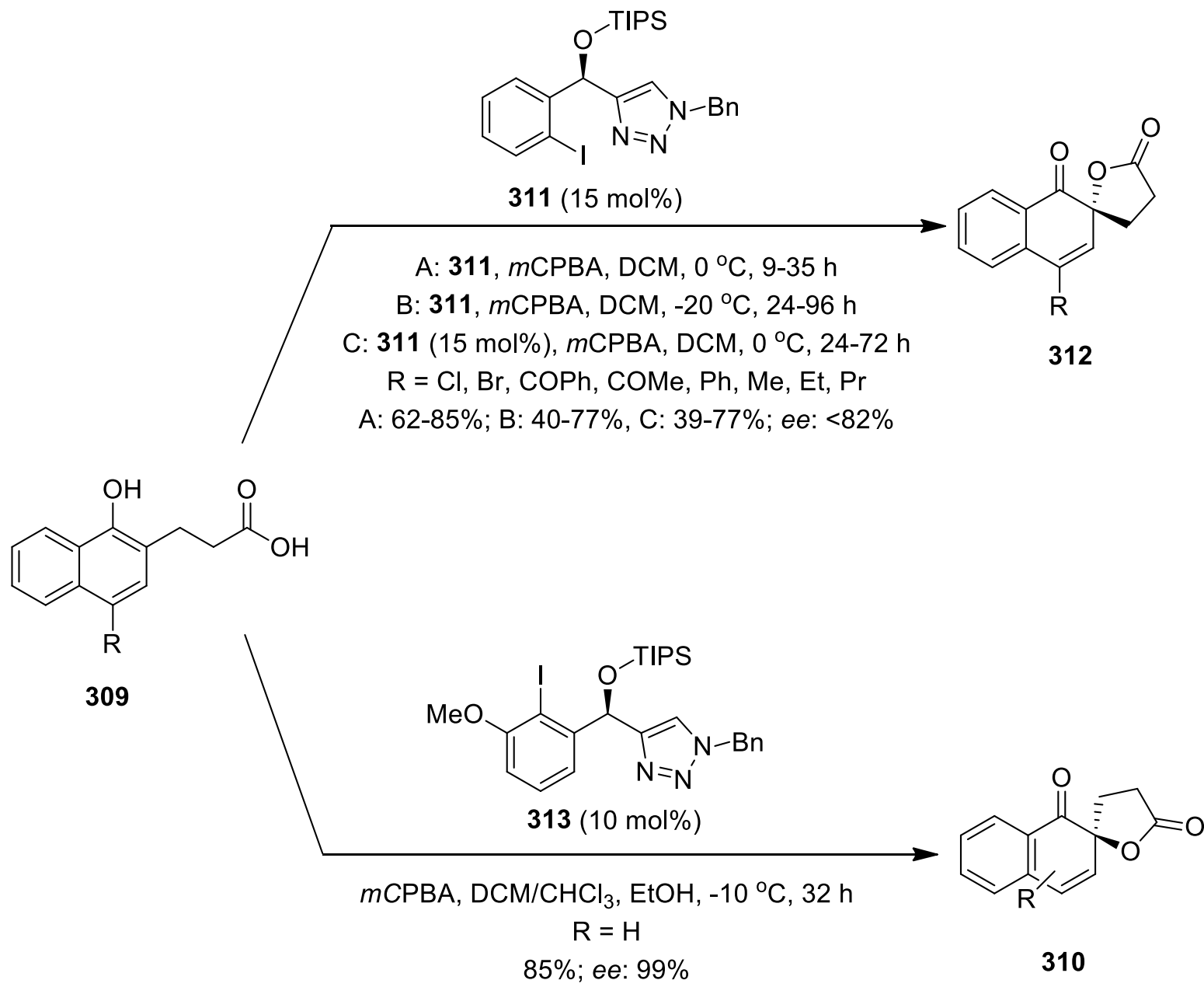

Scheme 84. Synthesis of spirolactones $\mathbf{3 1 2}$ and $\mathbf{3 1 0}$ using catalytic amount of chiral iodoarene $\mathbf{3 1 1}$ and $\mathbf{3 1 3 .}$

Several groups designed novel chiral iodoarene reagents for the asymmetric Kita-spirolactonization. For instance, Ogasawara et al. synthesized conformationally rigid C2-symmetric atropisomeric chiral diiododiene 314 and successfully applied as chiral organocatalyst in the dearomatizing spirolactonization of 1-naphthols 309 to yield (S)-spirolactone $\mathbf{3 1 0}$ with ee up to $73 \%$ (Scheme 85 ). ${ }^{117}$ Further, Imrich and Ziegler prepared the first 
carbohydrate-based chiral aryl iodide catalyst $\mathbf{3 1 5}$ by condensing partially protected glucosides with iodoresorcinol via Mitsunobu reaction. ${ }^{118}$ This catalyst was further employed for the oxidative spriolactonisation of $\mathbf{3 0 9}$ to provide spirolactone $\mathbf{3 1 2}$ in 77\% yield with er up to 80:20. Later, Quideau's research group succeeded in constructing helicine-based chiral iodoarene catalyst $\mathbf{3 1 6}$ from inexpensive precursors (L)-(+)-tartaric acid and 4-methylstyrene. ${ }^{119}$ This novel chiral catalyst 316 served as catalyst for the dearomative spirolactonization of $\mathbf{3 0 9}$ to afford chiral spirolactones $\mathbf{3 1 2}$ with moderate selectivity. Notably, reaction catalyzed by catalyst $\mathbf{3 0 6}$ and $\mathbf{3 0 7}$ gives $(R)$-isomer $\mathbf{3 1 2}$.
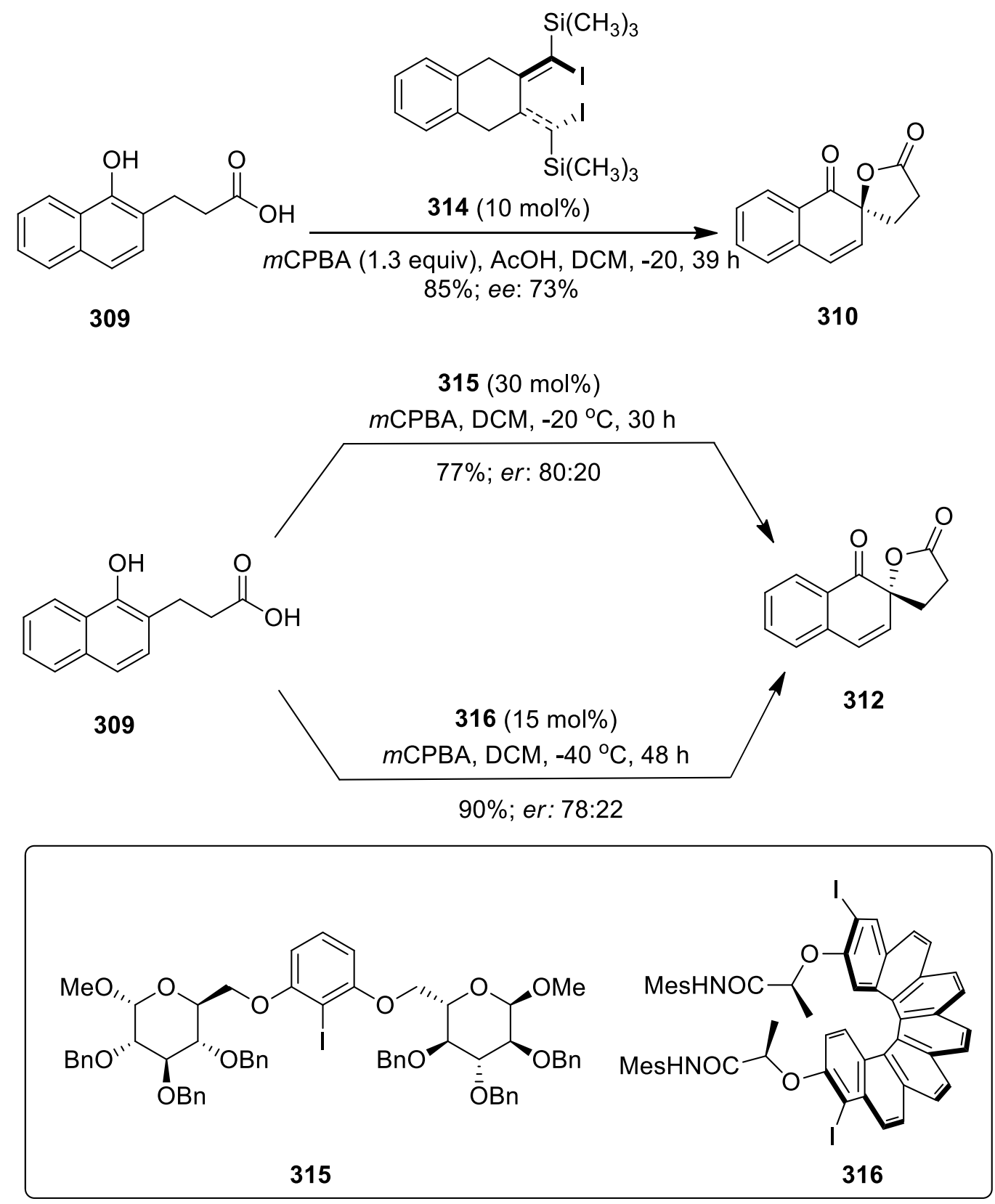

Scheme 85. Enantioselective synthesis of spirolactones 310 and 312 using iodoarenes 314-316 as precatalysts.

Very recently, $\mathrm{Phl}\left(\mathrm{OCOCF}_{3}\right)_{2}$-induced dearomatizative spirocyclization of various phenolic biarylic ketones 317 was by demonstrated by Wang's research team (Scheme 86). ${ }^{120}$ This is the first example employing ketone group as internal nucleophile for the spirocyclization reaction. Mechanistic details revealed formation of key 
intermediate exocyclic enol ether $\mathbf{3 1 9}$ that further undergoes PIFA-induced oxidation and C-C bond cleavage to yield cyclohexadienones 318. Notably, spiroannulation of ketonic substrates with long alkyl chain gave moderate yields. Moreover, biaryl substrates 317 bearing $\beta$-ketoester and aldehyde groups delivered corresponding spiroproducts albeit in low yields.<smiles></smiles>

317

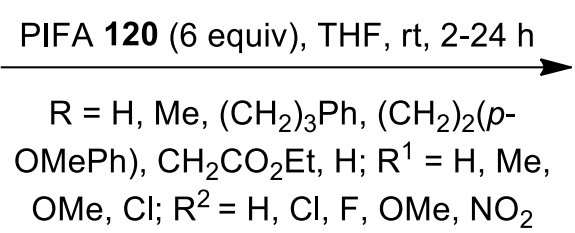

$20-86 \%$

Scheme 86. Synthesis of spirolactones 318 using PIFA 120 as an oxidant.

Zhao and co-workers performed the reaction of protected 3-hydroxy1,3-bis(2-hydroxyaryl)prop-2-en-1ones $\mathbf{3 2 0}$ with PIDA 6 that enabled synthesis of spiro-2,2'-benzo[b]furan-3,3'-ones 321 in quantitative yields at ambient temperature(Scheme 87). ${ }^{121}$ This cascade intramolecular spirocyclization process involves dual oxidative $\mathrm{C}-\mathrm{O}$ bond formation. A variety of substituents in both the phenyl rings were well tolerated.<smiles>[R]c1ccc([R6])c(C(O)=CC(=O)c2cc([R])ccc2O)c1</smiles>

320

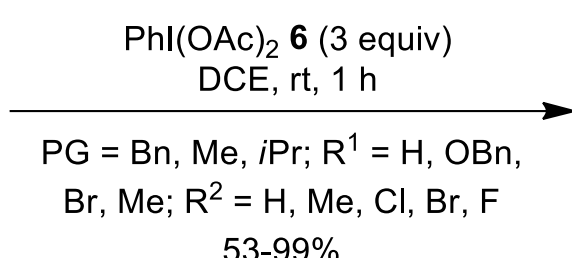

$53-99 \%$

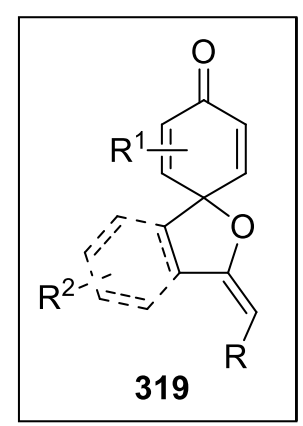

318<smiles>[R]c1ccc2c(c1)C(=O)C1(O2)Oc2ccc([R])cc2C1=O</smiles>

321

Scheme 87. Synthesis of spiro-2,2'-benzo[b]furan-3,3'-ones 321 using Phl(OAc) 6 as an oxidant.

Scheme 88 depicts the proposed mechanism for this cyclization reaction. Initially, nucleophilic attack of enolic oxygen of $\mathbf{3 2 0}$ at the iodine center of PIDA $\mathbf{6}$ generates intermediate 322, which cyclizes intramolecularly with the loss of $\mathrm{Phl}$ and acetate anion resulting in the formation of first $\mathrm{C}-\mathrm{O}$ bond. Intermediate $\mathbf{3 2 3}$ tautomerizes into $\mathbf{3 2 4}$ which later reacts with PIDA 6 to give intermediate $\mathbf{3 2 5}$. Then cyclization of $\mathbf{3 2 5}$ enables formation of second $\mathrm{C}-\mathrm{O}$ bond to furnish oxonium ion intermediate $\mathbf{3 2 6}$, which is finally attack by the acetate anion at the benzylic carbon resulting in the formation of spirocyclic product 321. ${ }^{121}$ 


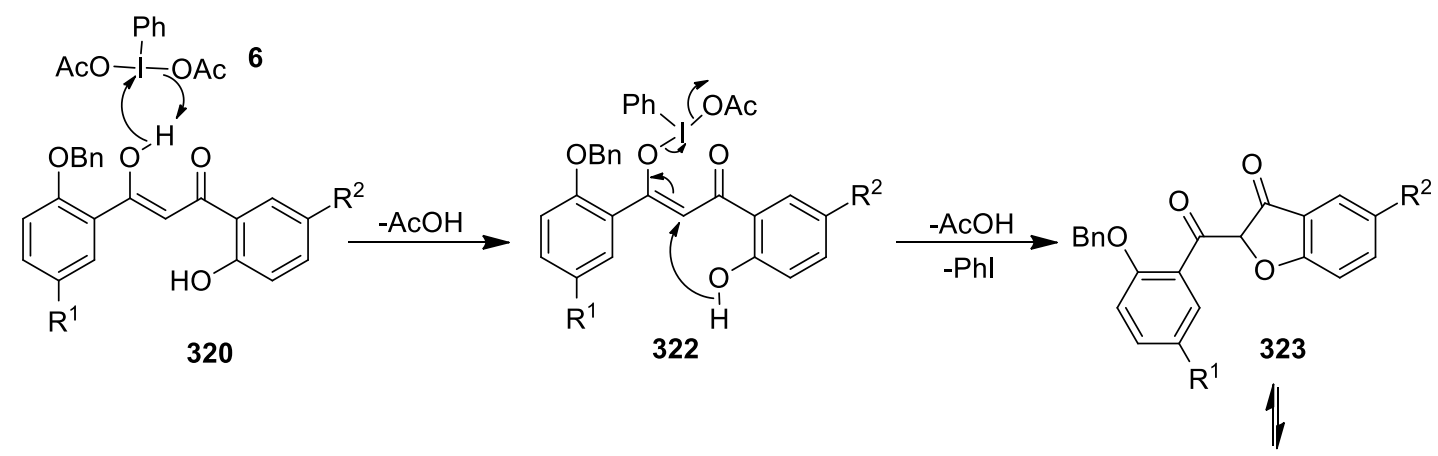

321

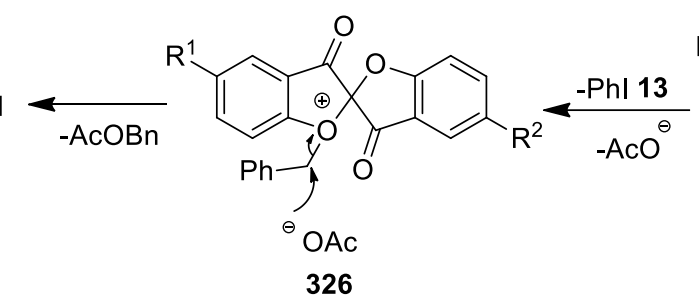<smiles>CC(C)(C)O[V](C)(C)c1ccccc1</smiles>

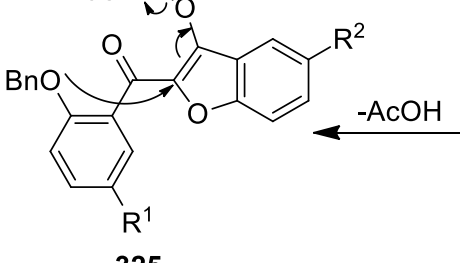

325<smiles></smiles><smiles>[R]c1ccc(OCc2ccc([R4])cc2C(=O)c2oc3ccc([R])cc3c2Cl)cc1</smiles>

Scheme 88. The plausible mechanism for the synthesis of spiro-2,2'-benzo[b]furan-3,3'-ones 321 using $\mathrm{Phl}(\mathrm{OAc})_{2} 6$ as an oxidant.

Meanwhile, Ciufolini and co-workers disclosed catalytic, enantioselective intramolecular oxidative cyclization of naphtholic alcohols $\mathbf{3 2 7}$ promoted by newly designed chiral aryl iodide $\mathbf{3 2 8}$ and $m$ CPBA (Scheme 89). ${ }^{122}$ Using the present cycloetherification process, an efficient synthesis of spirocyclic products 329 bearing different substituents were achieved in high yields (ee upto 98\%). Interestingly, presence of chiral center nearer to the $\mathrm{H}$-bonding amido group in $\mathbf{3 2 8}$ was found useful for effective optical induction. Also, asymmetric oxidative cyclization of naphtholic sulphonamide was accomplished using catalyst $\mathbf{3 2 8}$ under identical conditions.<smiles>[R]c1cc(CC([R])([R])CO)c(O)c2ccccc12</smiles>

327

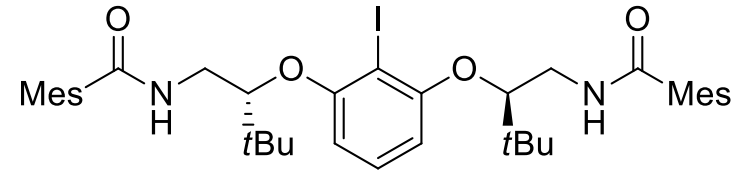

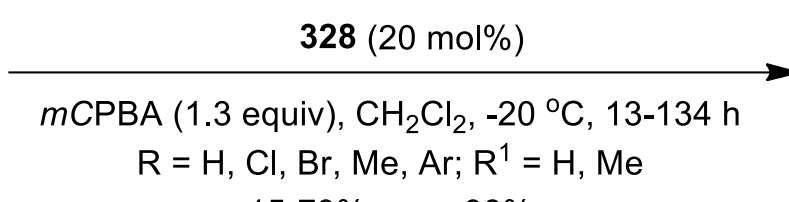

$15-79 \%$; ee: $<98 \%$<smiles>[R]C1=CC2(CC([R])([R])CO2)C(=O)c2ccccc21</smiles>

329

Scheme 89. Enantioselective synthesis of spirocyclic products $\mathbf{3 2 9}$ using chiral aryl iodide $\mathbf{3 2 8}$ as precatalyst.

Very recently, Deng et al. have reported a synthesis of spiro-ethers 332 via ring-opening/dearomatization of $9 \mathrm{H}$-fluoren-9-ol derivatives 330 promoted by iodosobenzene $331 .{ }^{123} \mathrm{~A}$ variety of substituents on the 9-aryl ring were well tolerated. Reaction occurs under mild condition with excellent substrates scope, regio- and diastereochemistry (Scheme 90). 
<smiles>[R]c1[R4]ccc2c1-c1c([R])cccc1C2(O)[Al]</smiles>

330

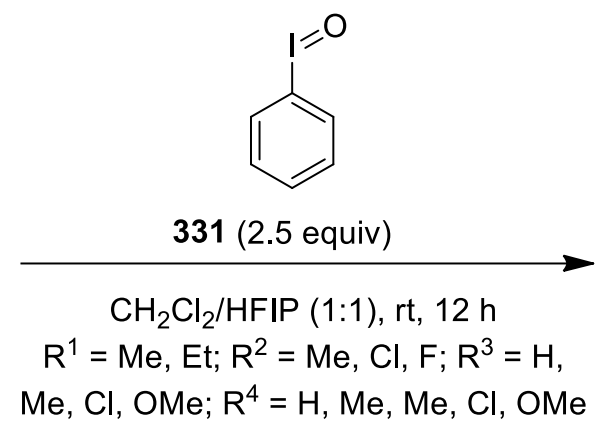

$37-99 \%$

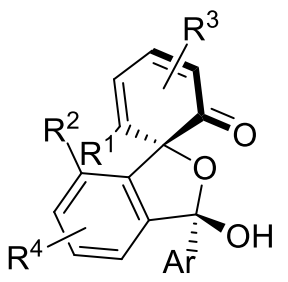

332

Scheme 90. Synthesis of oxo-spiro scaffolds $\mathbf{3 3 2}$ promoted by iodosobenzene 331.

A plausible mechanism for this transformation is depicted in Scheme 91. Reaction begins with the interaction of substrate $\mathbf{3 3 0}$ with iodosobenzene $\mathbf{3 3 1}$ in HFIP to form alkoxyliodine(III) intermediate $\mathbf{3 3 3 ,}$ following which $\beta$-carbon cleavage produces diaryliodonium salt $\mathbf{3 3 4}$. Reductive elimination of 334 provides oxygenated intermediate $\mathbf{3 3 5}$ with the loss of $\mathrm{Phl}$ 13. Further $\mathbf{3 3 5}$ reacts with $\mathrm{Phl}=\mathbf{0} \mathbf{3 3 1}$ to form intermediate 336, which undergoes nucleophilic attack by carbonyl group and subsequent dearomatization to give $1 H$ isobenzofuran-2-ium type product 338. Finally, nucleophilic attack by water delivers the oxo-spiro compound 332 as a cis-isomer. Notably, the hydrogen bonding between the carbonyl group and $\mathrm{H}_{2} \mathrm{O}$ probably accounts for the high diastereochemistry. ${ }^{123}$

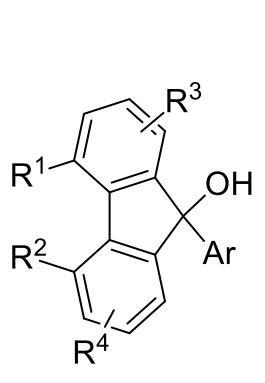

330

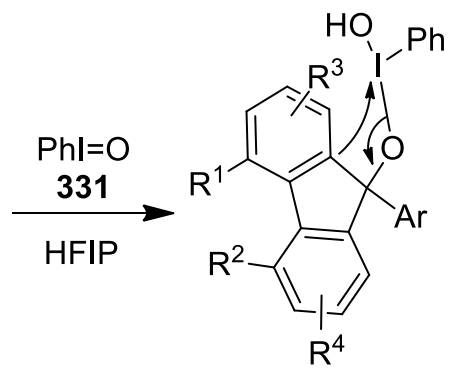

333<smiles>[R][X]c1cccc(C(=O)O)c1-c1c([R])cccc1I(O)P</smiles>

334

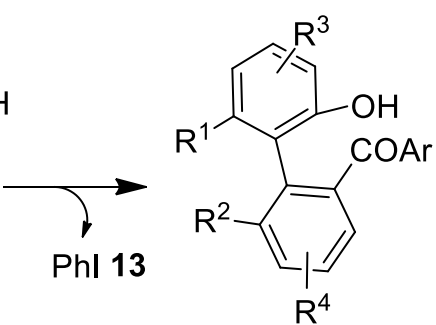

335

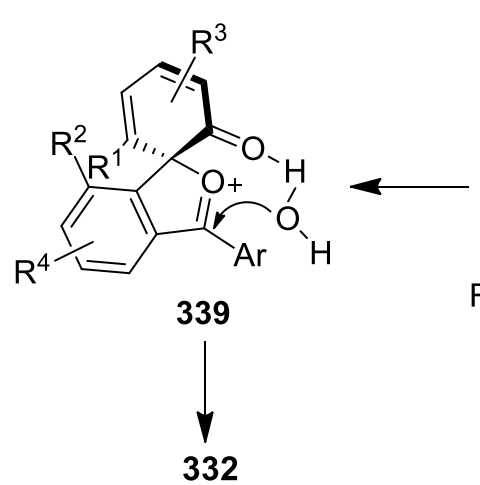

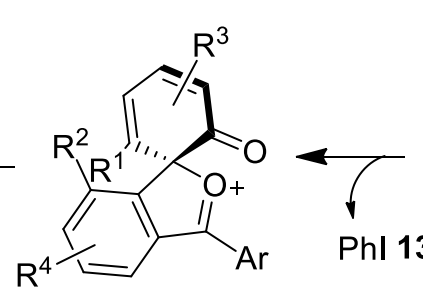

338

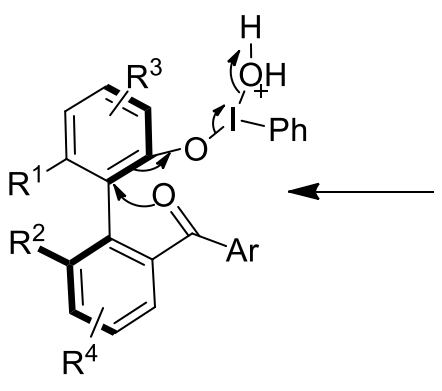

337<smiles>[R][X]c1ccc(C(=O)OCc2ccccc2)c(-c2c([R])cccc2[R])c1[R]</smiles>

336

Scheme 91. The proposed mechanism for the synthesis of oxo-spiro scaffolds $\mathbf{3 3 2}$ promoted by 331. 
Very recently, Tariq and Moran synthesized spirooxazolines 342 via oxidative dearomatization of amidetethered phenols $\mathbf{3 4 0}$ facilitated by active $\lambda^{3}$-iodane generated in-situ from $4-\mathrm{MeC}_{6} \mathrm{H}_{4} \mathrm{I} \mathbf{5 1} / \mathrm{m}$-CPBA catalytic system (Scheme 92). ${ }^{124}$ Authors predicted that the $\lambda^{3}$-iodane would activate the phenolic oxygen to form intermediate $\mathbf{3 4 1}$ and subsequent cyclization of pendent amide on to the aromatic ring results in the formation of desired product 342. Scope of the reaction was investigated with a range of aryl, alkyl and heteroaryl amidebased phenols under optimized conditions. Additionally, oxidative dearomatization of naphthol derivatives $\mathbf{3 4 3}$ yielded spirocycles $\mathbf{3 4 4}$ in moderate yields using 40 mol \% of 4-iodotoluene $\mathbf{5 1}$. Moreover, synthetic utility of this approach in the preparation of dihydrooxazines was successfully demonstrated.
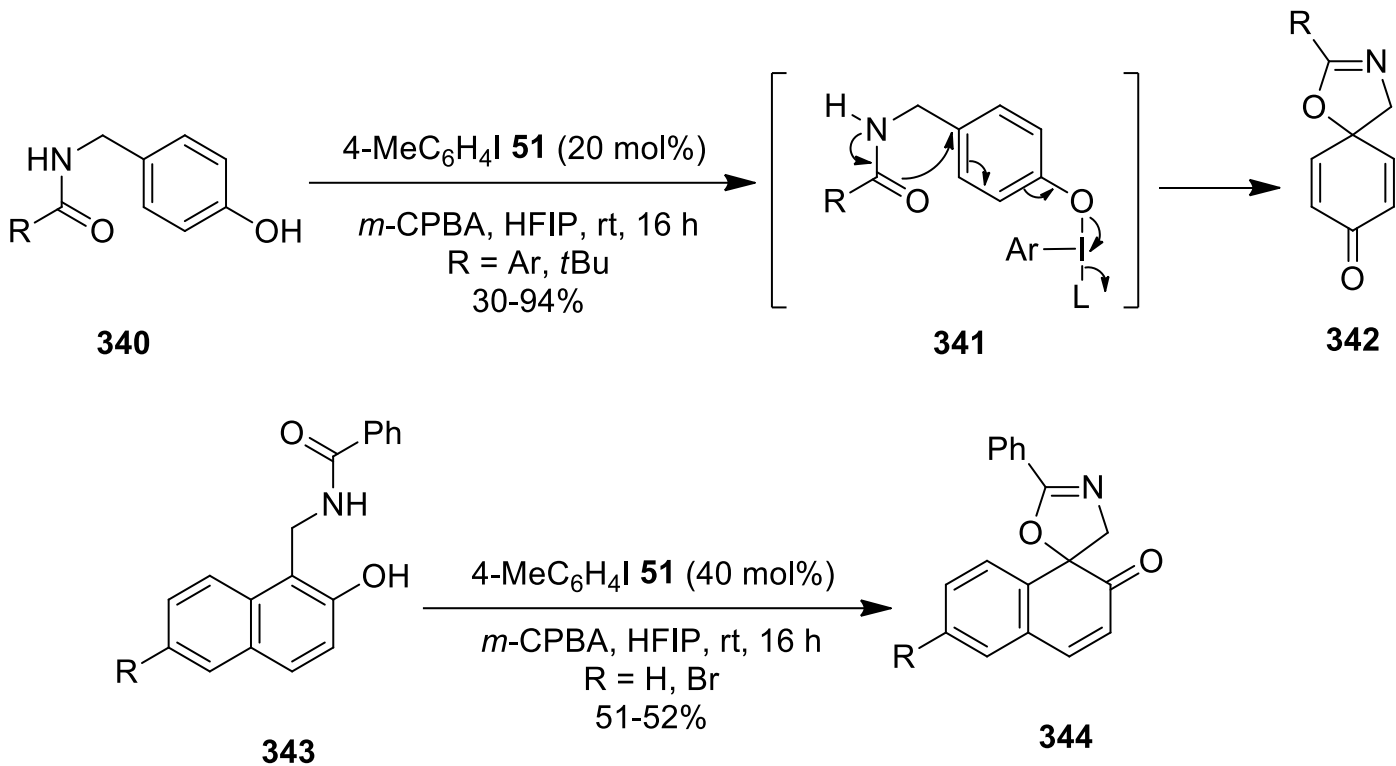

Scheme 92. Synthesis of spirooxazolines $\mathbf{3 4 2}$ using 4-iodotoluene $\mathbf{5 1 .}$

Cai and co-workers demonstrated synthesis of $\mathrm{N}$-fused spirolactams 345 from corresponding 3-arylpropanamides 232 via an asymmetric desymmetrization strategy (Scheme 93). ${ }^{95}$ The protocol was catalyzed by hypervalent iodine(III) species generated in situ from chiral precatalyst diiodospirobiindane $\mathbf{2 3 3}$ in the presence of $m C P B A$ as the terminal oxidant. para-substituted substrates $\mathbf{2 3 2}$ with halide or -OR groups smoothly underwent cyclization reaction to deliver products in high yields and moderate to good enantioselectivities.

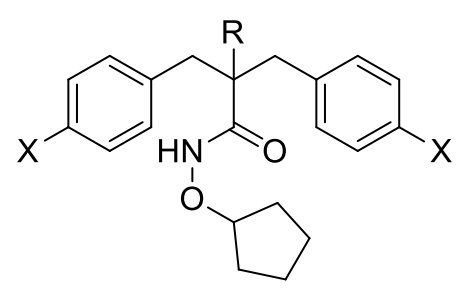

232
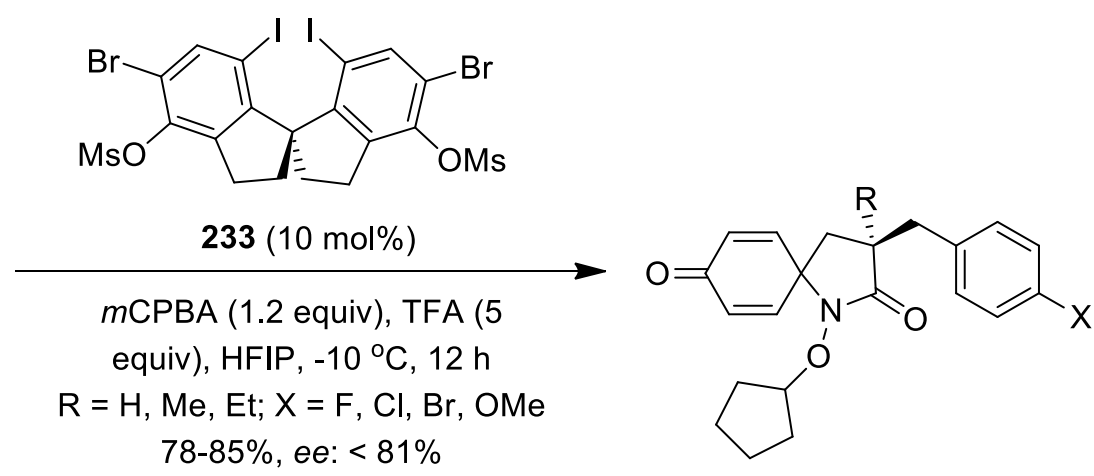

345

Scheme 93. Synthesis of spirolactams $\mathbf{3 4 5}$ using 233 as precatalyst. 
Odagi et al. constructed spirocyclic guanidines 348 from guanidine phenols 346 via dearomative spiroguanidination strategy by using oxidant 4-chloro-1-(diacetoxyiodo)benzene 347 (Scheme 94). ${ }^{125}$ The reaction was performed in 2,2,2,3,3,3-hexafluoro-2-propanol (HFIP) and trichloroethoxysulfonyl (Tces) group was found a suitable protecting group for the guanidine. In the proposed mechanism, phenol reacts with PIDA 6 to generate key aryl- $\lambda^{3}$-iodane intermediate 349, which is further attacked by the guanidine moiety at the para-position providing desired spiroguanidine $\mathbf{3 4 8}$ via dearomatization process. Additionally, orthospiroguanidination of substrate $\mathbf{3 5 0}$ yields spiroguanidine $\mathbf{3 5 1}$ under similar conditions.
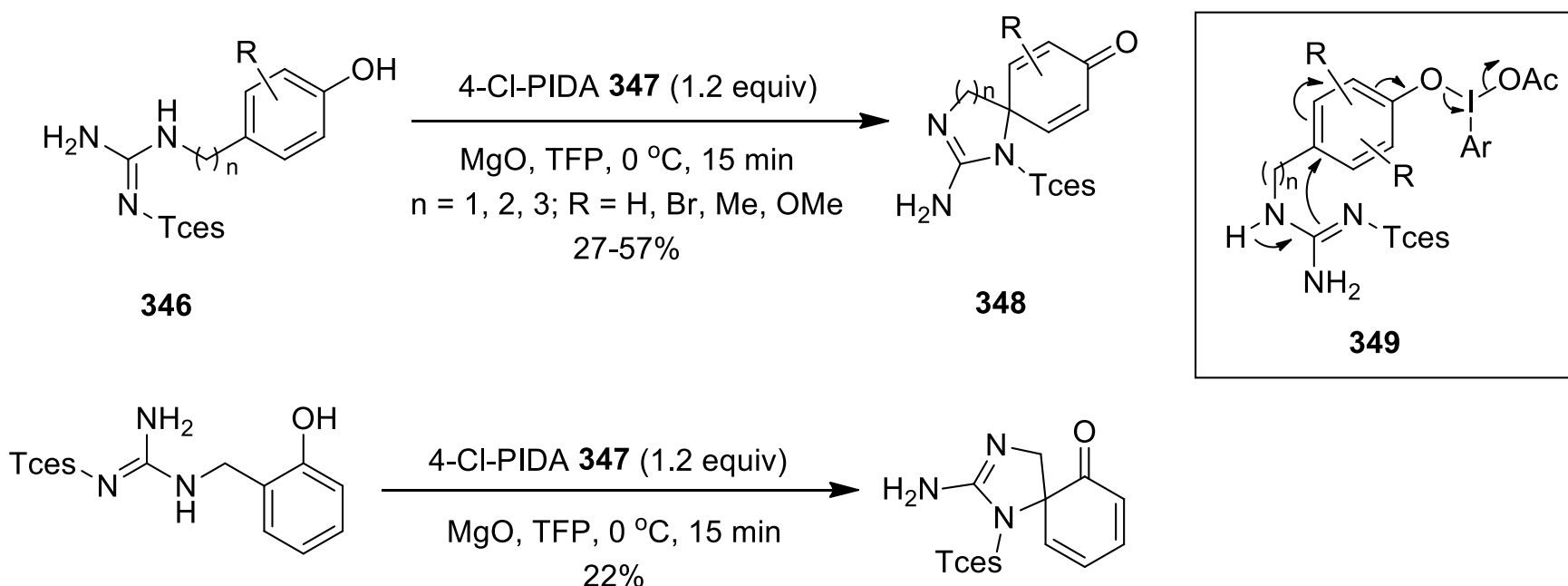

350

351

Scheme 94. Preparation of spiroguanidine derivatives 348 and 351 using 4-chloro-1-(diacetoxyiodo)benzene 347 as an oxidant.

\section{Hypervalent lodine-Mediated Late-Stage Functionalization of Heterocycles}

Direct functionalization of heterocycles using hypervalent iodine reagents is fast-growing field in organic chemistry. These reagents find profound applications in the functionalization of variety heterocyles via synthetic transformations such as oxidative amination, alkylation, acetoxylation, halogenation, etc. In this section, all recent developments acheived in this area will be covered.

\subsection{Amination/azidation of heterocycles}

In 2017, Mondal et al. disclosed PIDA-induced intermolecular oxidative $\mathrm{C}\left(\mathrm{sp}^{2}\right)-\mathrm{H}$ amination of imidazopyridines 352 (Scheme 95). ${ }^{126}$ Various cyclic amines 353 such as piperidine, morpholine and thiomorpholine reacted smoothly with imidazo[1,2-a]pyridines 352 to provide 3-amino substituted imidazopyridines 354 at room temperature. Moreover, regioselective $\mathrm{C}-\mathrm{H}$ amination of indolizines was achieved under identical conditions. 


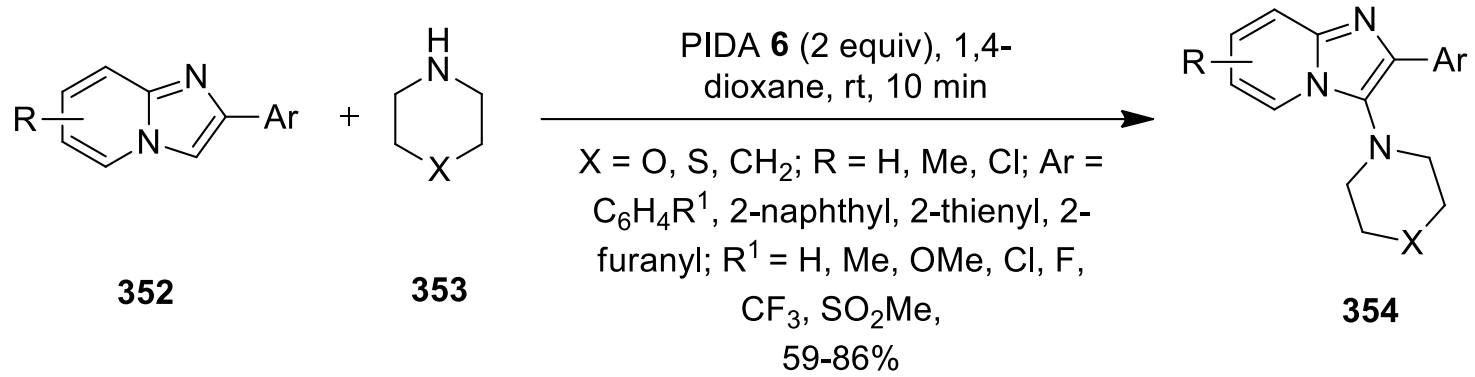

Scheme 95. PIDA-mediated reaction of imidazopyridines $\mathbf{3 5 2}$ with cyclic amines 353.

Based on the experimental results, the proposed mechanism likely follows radical pathway as depicted in Scheme 96. Reaction of cyclic amine $\mathbf{3 5 3}$ with PIDA $\mathbf{6}$ forms $N$-iodoamido species $\mathbf{3 5 5}$ which gives radical $\mathbf{3 5 6}$ that further reacts with imidazo[1,2-a]pyridine $\mathbf{3 5 2}$ to furnish radical intermediate $\mathbf{3 5 7}$. Finally, product $\mathbf{3 5 4}$ was obtained through the loss of $\mathrm{AcOH} .{ }^{126}$

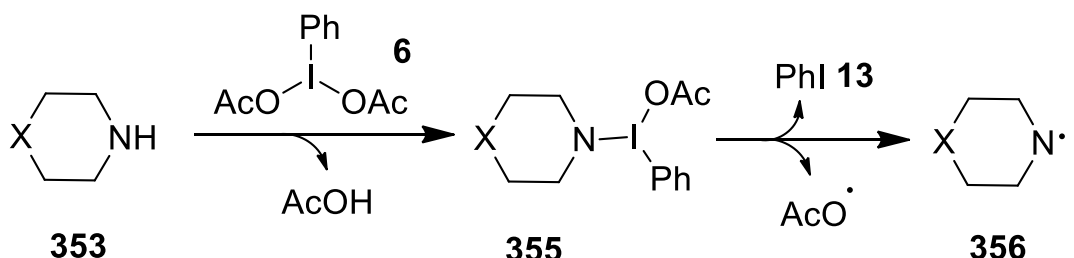

355

356

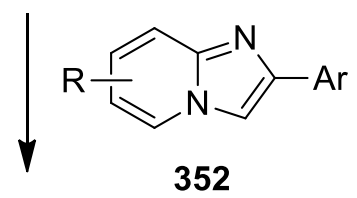<smiles>[X]CCN(CC[X])c1c([Al])nc2cc[R]:[X]c12</smiles>

354

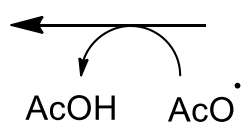<smiles>[X]CCN(CC[R])C1C(Br)N=C2C=CC=CN21</smiles>

357

Scheme 96. The proposed mechanism for the synthesis of 3-amino substituted imidazopyridines 354 using PIDA 6 as oxidant.

Later, in 2018 Su's research group performed cross-dehydrogenative coupling of $\alpha-C\left(\mathrm{sp}^{3}\right)-\mathrm{H}$ bond of substrates 359 with azoles 358 using sole oxidant PIDA 6 (Scheme 97). ${ }^{127}$ This protocol provides an easy access to a variety of $\mathrm{N}$-alkylated azoles $\mathbf{3 6 0}$ by employing ethers, tetrahydrothiophene or $\mathrm{N}$-Methyl-2-pyrrolidone as coupling reagents. Further synthetic utility was demonstrated by performing a gram scale reaction, which extends the practicality of this oxidative coupling reaction. Reaction follows radical pathway as indicated by various radical-trapping experiments. 


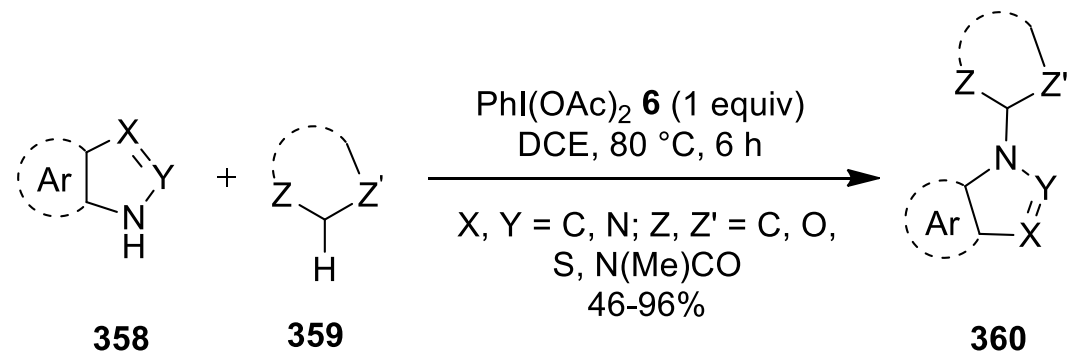

Scheme 97. PIDA-mediated $\mathrm{C}\left(\mathrm{sp}^{3}\right)-\mathrm{H}$ amination of substrates 359 with azoles 358.

In 2018, an interesting one-pot protocol for the iodoarylation of NH-pyrazoles 361 with aryliodine diacetates 362 was developed by Cheng and co-workers (Scheme 98). ${ }^{128}$ This reaction proceeds through hypervalent iodine-induced oxidation of $\mathbf{3 6 1}$ generating pyrazole-4-arylliodonium tosylate, [Arl(pyrazole)][OTs] 364 in situ which undergoes facile deprotonation forming zwitterionic iodonium ylide $\mathbf{3 6 5}$. Finally, an intermolecular $N$ arylation mediated by 1,10-phenanthroline/ $\mathrm{K}_{2} \mathrm{CO}_{3}$ yields the expected 1,4-disubstituted pyrazoles 363 . Further, the proposed intermediate $\mathbf{3 6 5}$ could be easily prepared and transformed into the target the iodoarylation product in high yield.

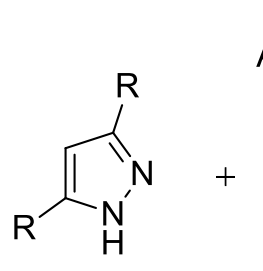

361<smiles>[R]c1ccc(I(OC(C)=O)OC(C)=O)cc1</smiles>

362

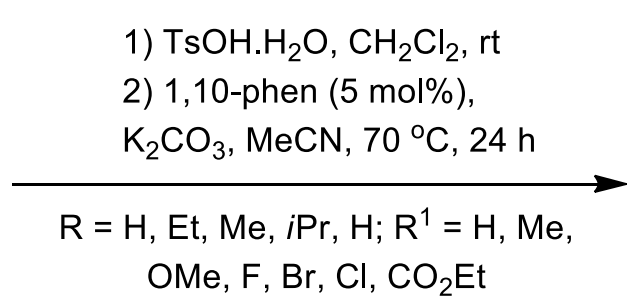

$30-79 \%$

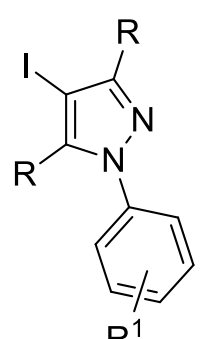

$\mathrm{R}^{1}$

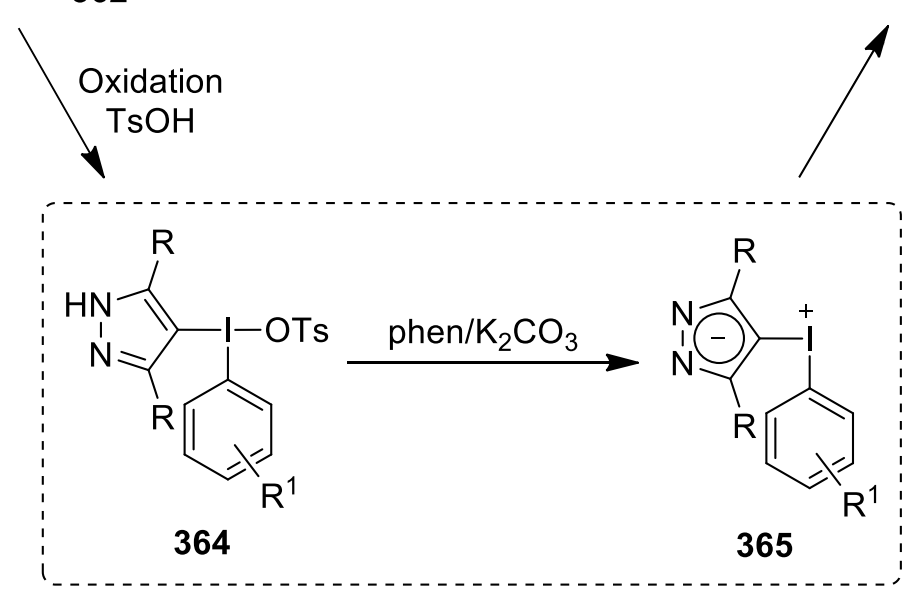

Scheme 98. lodoarylation of various pyrazoles 361 using aryliodine diacetates 362 as coupling partner.

Further, radical-based strategy to prepare 3-azido-2-oxindoles $\mathbf{3 6 8}$ was developed by Chen et al. via $\mathrm{C}\left(\mathrm{sp}^{3}\right)-\mathrm{H}$ azidation of 3-substituted-2-oxindoles 366 (Scheme 99). ${ }^{129}$ This transformation employs TMSN 367 as the azide reagent in the presence of $\mathrm{PhI}(\mathrm{OAc})_{2} 6$ and $\mathrm{Et}_{3} \mathrm{~N}$ as an oxidant and additive respectively. Notably, azidation reaction proceeds smoothly with 3-aryl-2-oxindoles whereas 3-alkyl-2-oxindole showed no reactivity. 


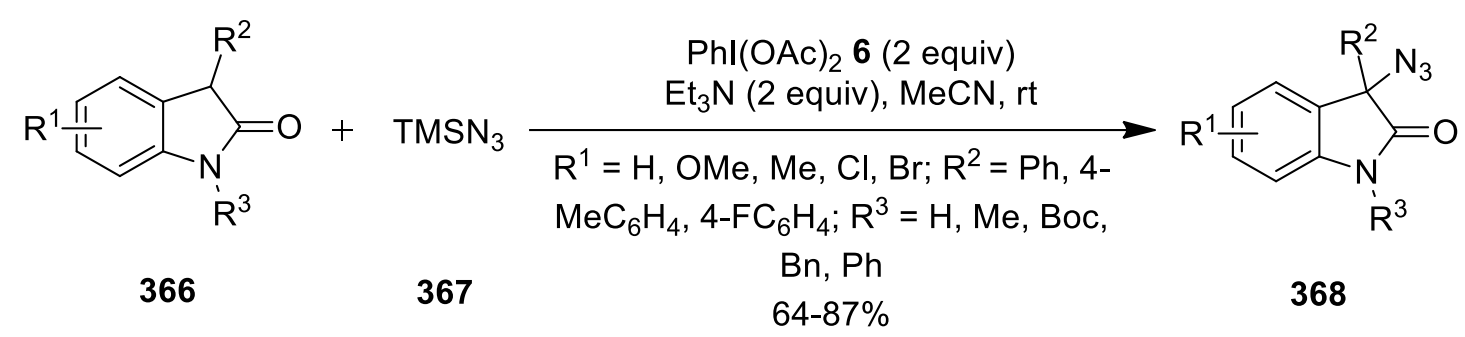

Scheme 99. PIDA-induced synthesis of 3-azido-2-oxindoles 368 through $\mathrm{C}\left(\mathrm{sp}^{3}\right)-\mathrm{H}$ azidation of 3-substituted-2oxindoles 366.

\subsection{Alkylation/Alkynylation of Heterocyles}

In 2017, Zhang's research group demonstrated PIDA-mediated C-H perfluoroalkylation of 8-aminoquinoline amides 369 to yield perfluoroalkylated quinolones 371 (Scheme 100). ${ }^{130}$ Reaction scope was administered by using different perfluoroalkyl sources 370 such as $T_{M S C} F_{5}, T M S n-C_{3} F_{7}$ and $T M S C F_{3}$. Based on the various control experiments, it was confirmed that reaction proceeds via single electron transfer mechanism.

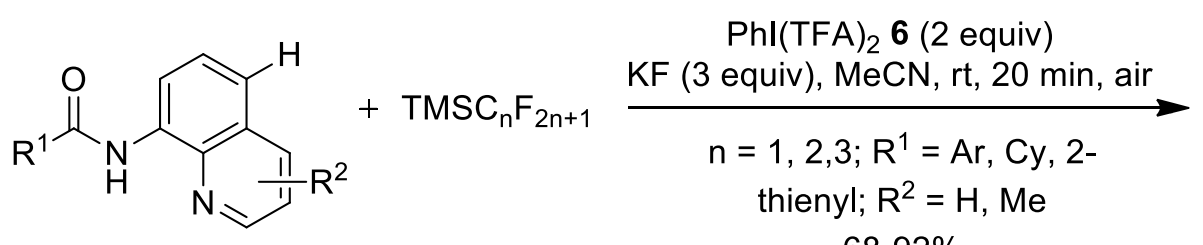

369<smiles>[R]C(=O)Nc1ccc(C(F)F)c2c([R])ccnc12</smiles>

371

Scheme 100. PIDA-mediated C-H perfluoroalkylation of 8-aminoquinoline amides 369.

Later, Maruoka and co-workers employed [bis(difluoroacetoxy)iodo]benzene $\mathbf{3 7 3}$ as the difluoromethylating agent for the $\mathrm{C}-\mathrm{H}$ difluoromethylation of heteroarenes $\mathbf{3 7 2}$ (Scheme 101). ${ }^{131}$ This reaction involves photolytic cleavage of iodine(III) reagent 373 on exposure to visible light $(\lambda=400 \mathrm{~nm}$ ) generating difluoromethyl radical via decarboxylation that would react with heteroarenes $\mathbf{3 7 2}$ to deliver difluoromethylated products 374 . A series of heteroarenes $\mathbf{3 7 2}$ such as pentoxifylline, uraciles, pyridines, pyridazine, pyrimidines, triazine, pyrazine and pyrazole smoothly reacted under the optimized reaction conditions.

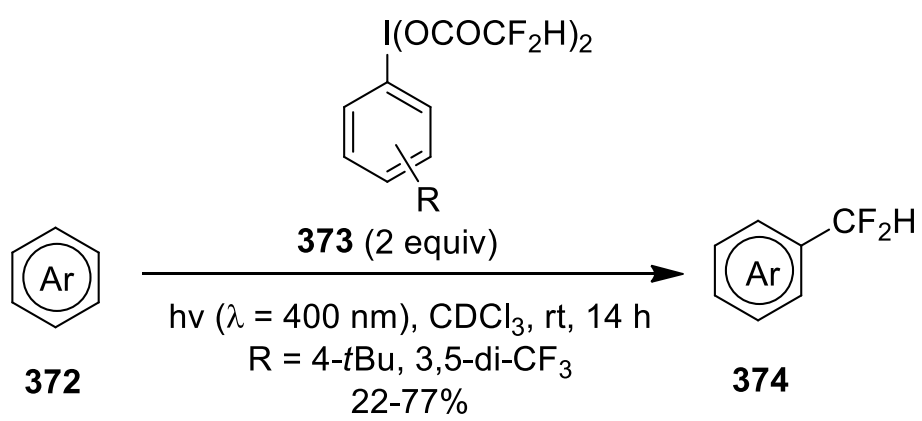

Scheme 101. C-H difluoromethylation of heteroarenes $\mathbf{3 7 2}$ using [bis(difluoroacetoxy)iodo]benzene $\mathbf{3 7 3}$ as difluoromethylating agent. 
A regioselective $\mathrm{C} 2$-alkylation of $\mathrm{N}$-heteroaromatic $\mathrm{N}$-oxides $\mathbf{3 7 5}$ using tert-/sec-alkyl alcohol $\mathbf{3 7 6}$ as an alkylating reagent has been reported by Sen and Ghosh (Scheme 102). ${ }^{132}$ This PIDA-promoted reaction involves formation of intermediate 378, which upon homolytic C-C bond cleavage of alcohols (via SET pathway), followed by alkylation and final aromatization to deliver 2-alkylated products $\mathbf{3 7 7}$ in useful yields.

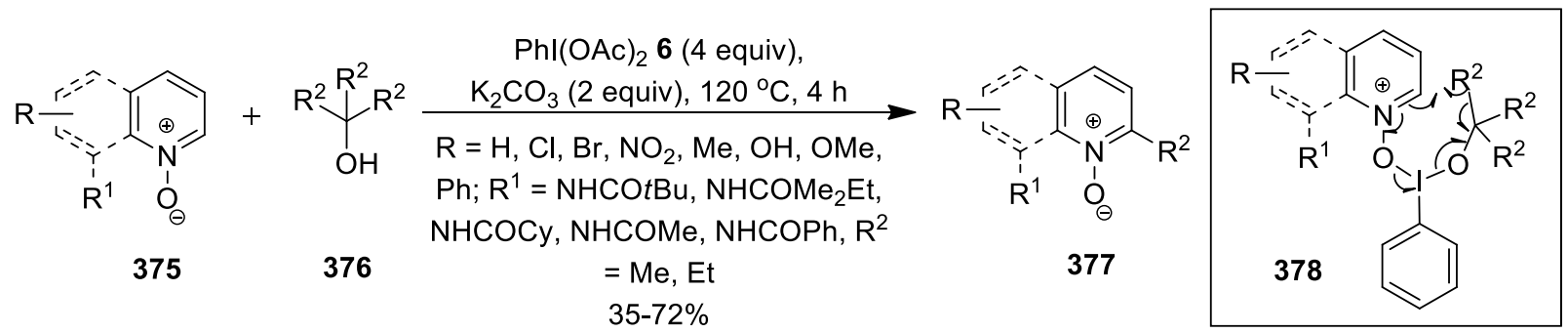

Scheme 102. PIDA-promoted C2-alkylation of N-heteroaromatic $N$-oxides $\mathbf{3 7 5}$ using secondary/tertiary alcohols 376 as an alkylating reagent.

Frenette's team developed a photoredox protocol featuring visible light-induced C-H alkylation of heteroaromatics $\mathbf{3 7 9}$ by using carboxylic acids $\mathbf{3 8 0}$ as coupling partner (Scheme 103). ${ }^{133}$ The present decarboxylative coupling method employs organic photocatalyst, 9-mesityl-10-methyl acridinium and oxidant PIFA 120. This catalytic system converts carboxylic acids $\mathbf{3 8 0}$ (primary, secondary and tertiary) into alkyl radicals that undergo radical substitution process to deliver corresponding alkylated products $\mathbf{3 8 1}$ in variable yields. Several heteroaromatic compounds $\mathbf{3 7 9}$ such as quinaldine, benzimidazole, benzothiazole, 2,6-dichloropurine, pyridines, pyrimidine, pyrazine and phthalazines were successfully tested under the optimized reaction conditions. Additionally, late-stage $\mathrm{C}-\mathrm{H}$ functionalization of drugs such as Voriconazole, quinine and Varenicline were also achieved in variable yields.

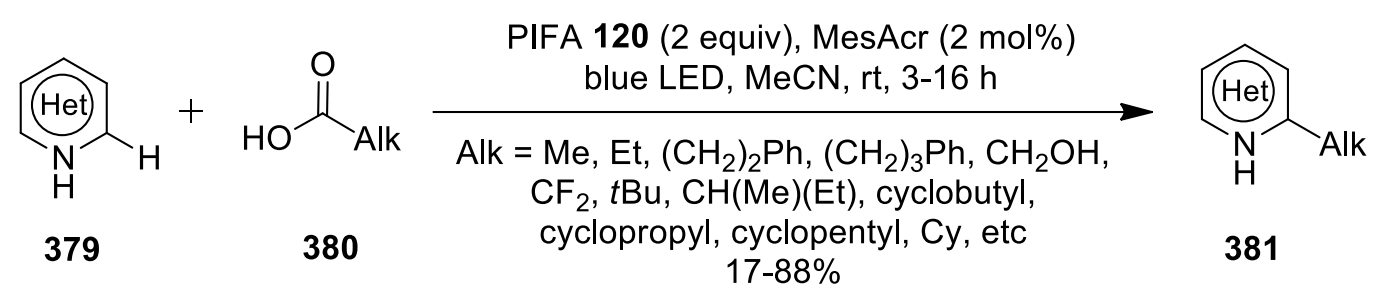

Scheme 103. PIDA-mediated $\mathrm{C}-\mathrm{H}$ alkylation of heteroaromatics $\mathbf{3 7 9}$ using carboxylic acids $\mathbf{3 8 0}$ as coupling partner.

A similar decarboxylative coupling protocol was developed for the $\mathrm{C}-\mathrm{H}$ alkylation of $\mathrm{N}$-heterocycles $\mathbf{3 7 9}$ with carboxylic acids $\mathbf{3 8 0}$ as C-centered radical source and PIFA 120 as the oxidant (Scheme 104). ${ }^{134} \mathrm{~A}$ variety of $\mathrm{N}$ heterocycles 379 including quinolone, isoquinoline, and pyridine derivatives were smoothly transformed into the corresponding C2-alkylated products 381. Moreover, decarboxylative $\mathrm{C}-\mathrm{H}$ alkylation of $\mathrm{N}$-heterocycles $\mathbf{3 7 9}$ with other carboxylic acid derivatives such as $\alpha$-oxocarboxylates $\mathbf{3 8 2}$ and alcohol 297-derived oxalates were also demonstrated under identical conditions. Very recently, Chen and co-workers disclosed similar photoredox catalysed $\mathrm{C}\left(\mathrm{sp}^{3}\right)-\mathrm{H}$ heteroarylation of aliphatic alcohols using perfluorinated hydroxybenziodoxole as an oxidant. ${ }^{135}$ 


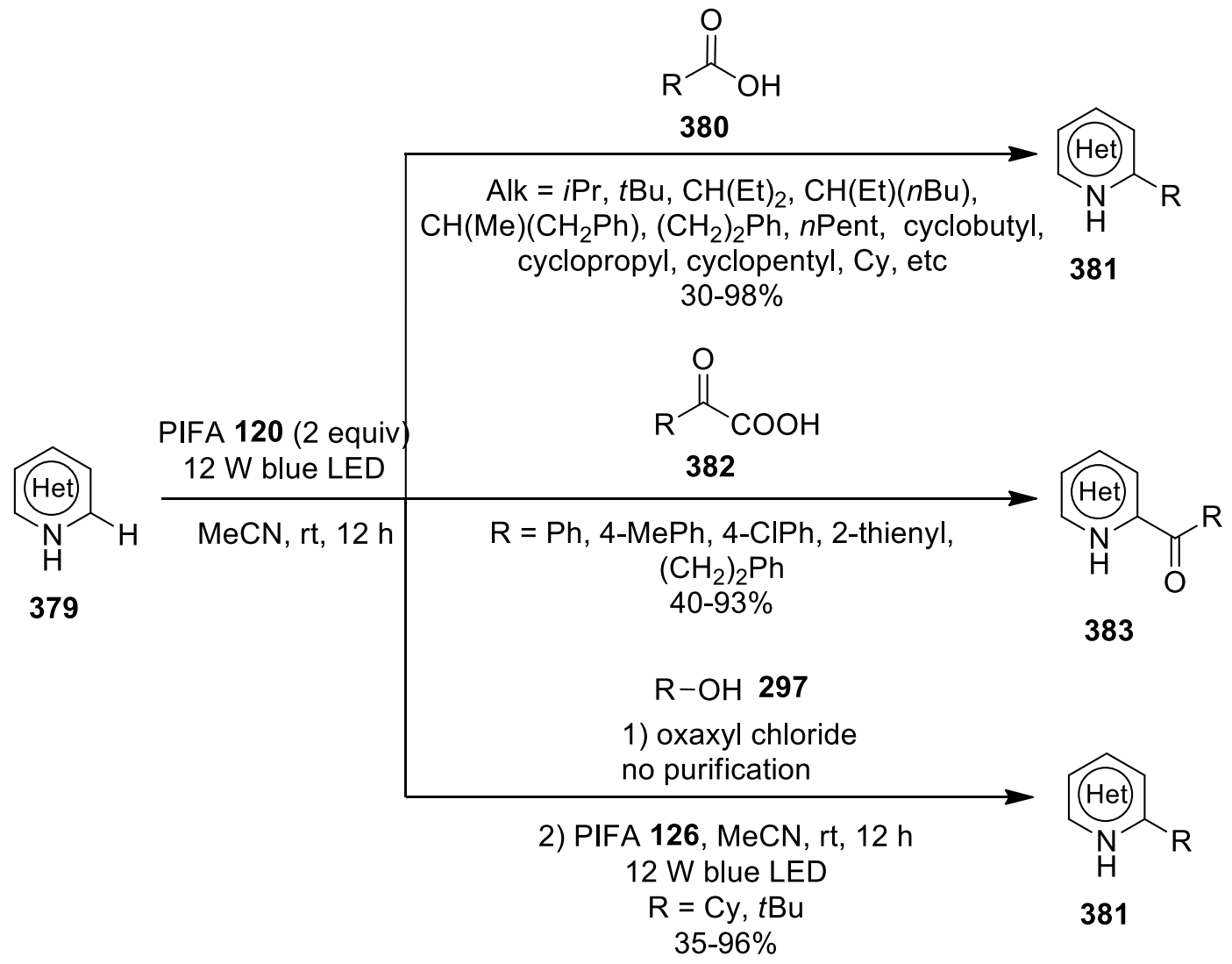

Scheme 104. PIDA-mediated C-H alkylation/acylation of $N$-heterocycles 379.

Using ethynyl-1,2-benziodoxol-3(1H)-one (EBX) 385 as an alkynylating reagent, Roy et al. carried out direct C3-alkynylation of 3-substituted-2-oxindoles $\mathbf{3 8 4}$ under metal-free conditions (Scheme 105). ${ }^{136}$ Reaction works efficiently on variety of 2-oxindole-3-alkylcarboxylates 384 providing anticipated 3-alkynyl-3-alkyl/aryl 2oxindoles $\mathbf{3 8 7}$ in significant yields. Further synthesized alkynylated products $\mathbf{3 8 7}$ were transformed into enantioenriched 2-oxindoles via Pd-catalyzed decarboxylative allylation in good yields with ee up to $96 \%$.
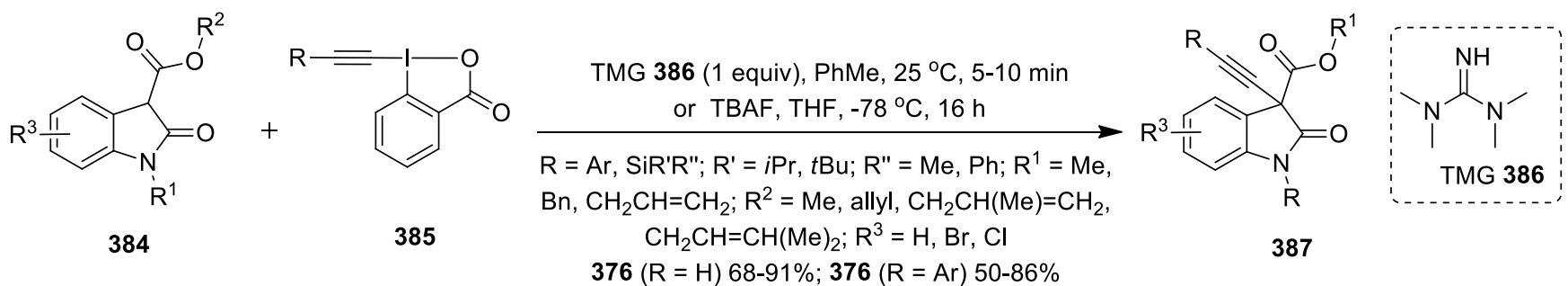

387

Scheme 105. Oxidative alkynylation of 3-substituted-2-oxindoles 384 using ethynyl-1,2-benziodoxol-3(1H)-one 385 as an alkynylating reagent.

\subsection{Alkoxylation and acetoxylation of heterocycles}

Kotagiri's group reported C-3 alkoxylation of simple oxindoles 388 via PIFA-mediated oxidative cross-coupling with different linear or branched alcohols 297 (Scheme 106). ${ }^{137}$ This reaction provides 3-alkoxyoxindoles 389 in $43-93 \%$ yields under mild conditions in shorter reaction time. Further using $\mathrm{PIFA} / \mathrm{I}_{2}$ system, in situ iodo- 
alkoxylation of oxindoles 388 resulted in the one-pot synthesis of 5-iodo-3-monoalkoxyoxindoles 390 or 5-iodo3,3-dialkoxyoxindoles 391 in appreciable yields.

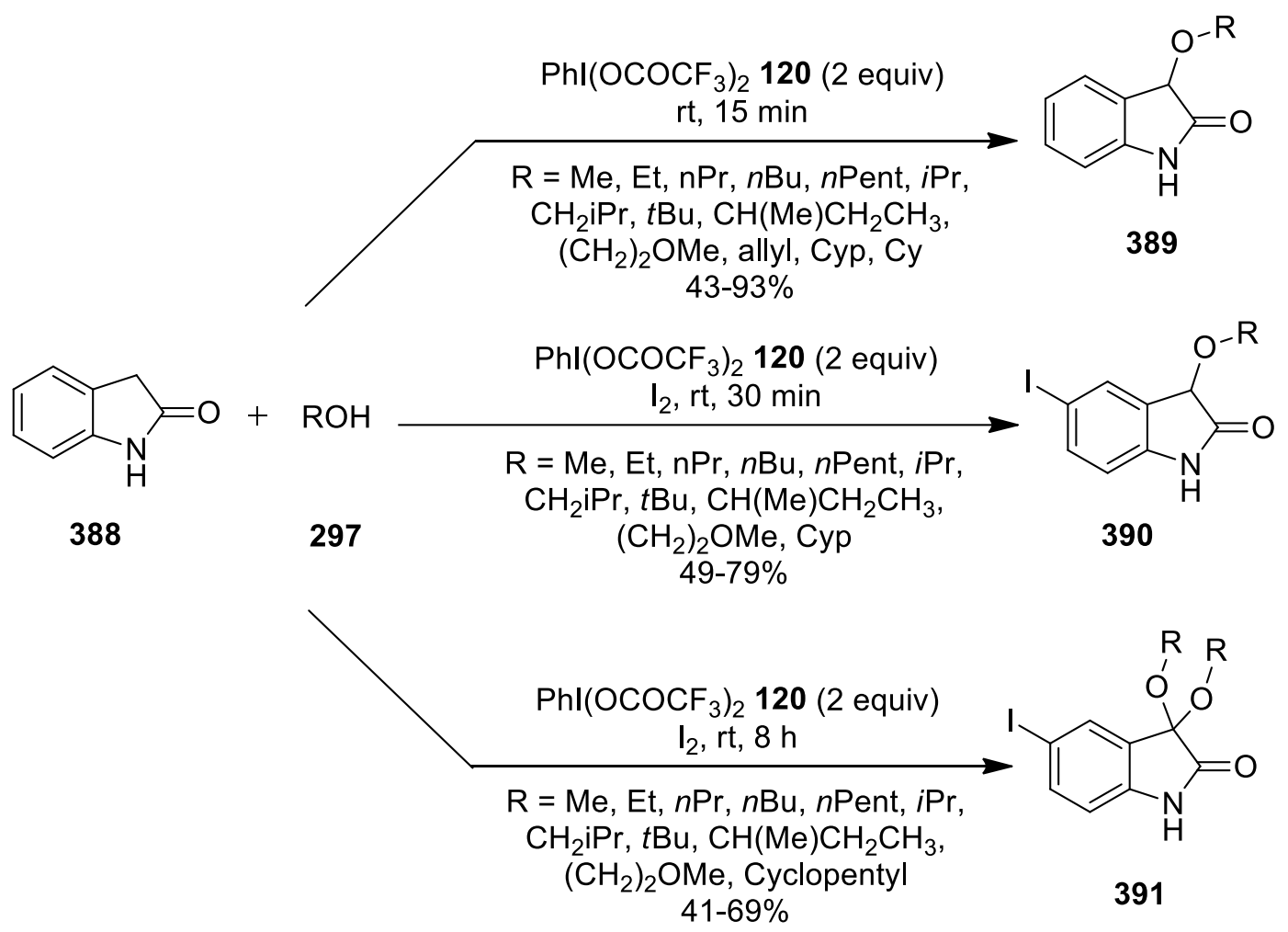

Scheme 106. PIFA-mediated oxidative cross-coupling of oxindoles 388 with alcohols 297.

Later, Majee's research group performed visible-light-promoted $\mathrm{C}\left(\mathrm{sp}^{3}\right)-\mathrm{H}$ acetoxylation of aryl-2H-azirines 392 using PIDA 6 as the reagent. ${ }^{138}$ Rose Bengal was used as the organophotoredox catalyst (Scheme 107). Reaction proceeds through radical pathway involving single electron transfer mechanism that requires presence of light irradiation. A library of acyloxylated azirines $\mathbf{3 9 3}$ was isolated in variable yields with excellent regioselectivity and functional group compatibility. Moreover, reaction occurs at room temperature under aerobic condition and applicable for gram scale synthesis.

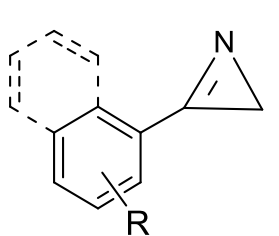

392

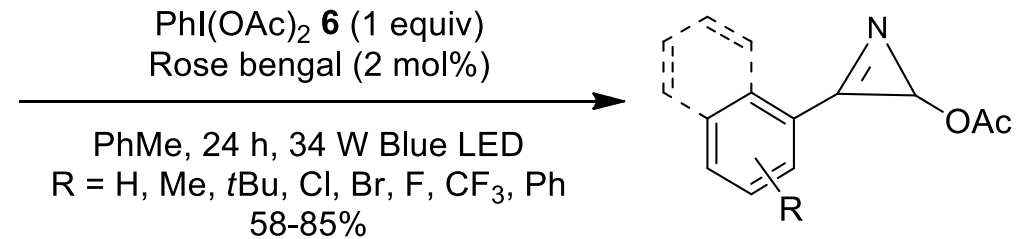

393

Scheme 107. PIDA-mediated $\mathrm{C}\left(s p^{3}\right)-\mathrm{H}$ acetoxylation of $2 \mathrm{H}$-azirines 392 using rose bengal as the organophotoredox catalyst.

Very recently, Kumar and co-workers transformed imidazo[1,2-a]pyridine 394 into the corresponding $N$ acetoxymethyl/alkoxymethyl-N-arylimidazo[1,2-a]pyridine-3-amines 396 via PIDA-induced [1,2]-ipso migration 
strategy (Scheme 108). ${ }^{139}$ This reaction was proposed to proceed via Wheland-type aziridine intermediate 395 which upon subsequent ring opening assisted by acetate/alkoxy nucleophile delivers rearranged products 396.

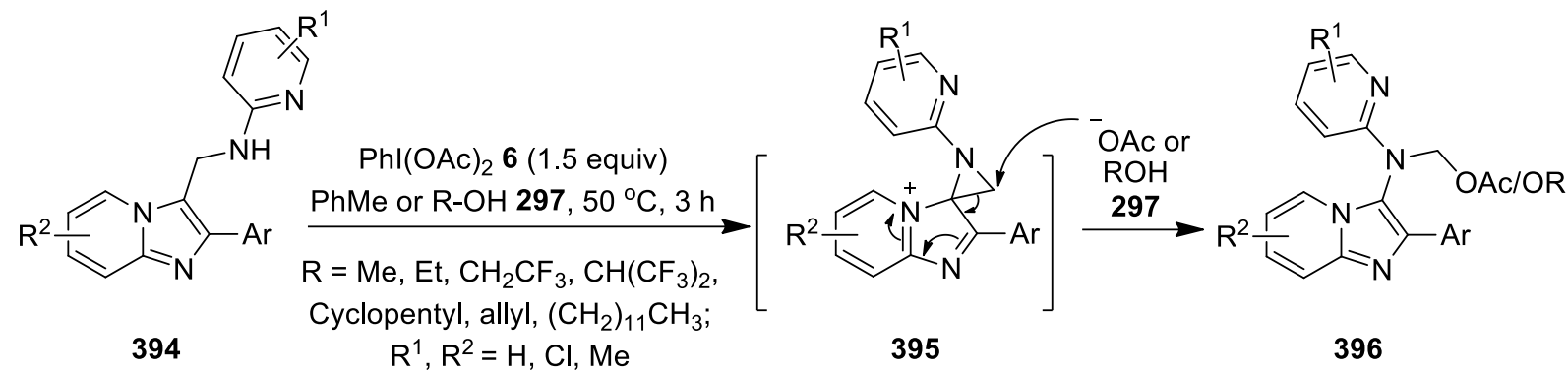

$35-93 \%$

Scheme 108. PIDA-promoted synthesis of $N$-acetoxymethyl-and $N$-alkoxymethyl- $N$-arylimidazo[1,2-a]pyridine3-amines 396.

\subsection{Halogenation/cyanation of heterocycles}

In 2018, a mild method for the selective $\mathrm{C}-\mathrm{H}$ halogenation of indoles 397 with $\mathrm{Phl}(\mathrm{OAc})_{2} 6 / \mathrm{NaX}$ system was developed by Rao and co-workers (Scheme 109). ${ }^{140}$ This method is applicable for the chlorination, bromination and iodination of functionally diverse indoles providing privileged scaffold, 3-haloindoles $\mathbf{3 9 8}$ in moderate to excellent yields. Reaction mechanism involves PIDA-mediated oxidation of $\mathrm{NaX}$ generating positive halogen species $\left(\mathrm{X}^{+}\right)$which is attacked by indole regioselectively at the C-3 position to form intermediate 399 and subsequent proton loss yields halo product 398.
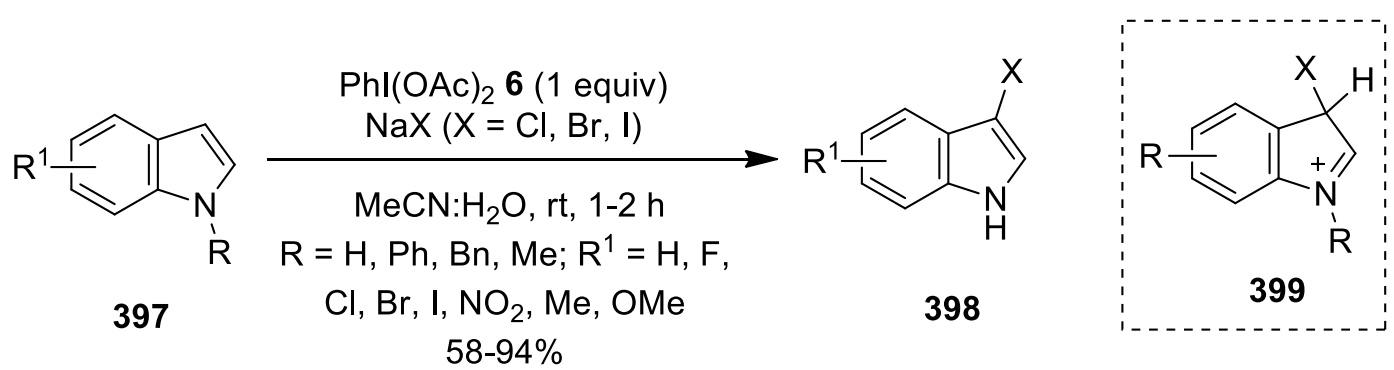

Scheme 109. PIDA-mediated $\mathrm{C}-\mathrm{H}$ halogenation of indoles 397 using NaX as the halide source.

Further, Indukuri et al. devised a regioselective protocol for the C-3 halogenation/thiocyanation of imidazo[1,2-a]pyridines/pyrimidine $\mathbf{4 0 0}$ by grinding with alkali metal/ammonium salts (M-X) mediated by PIDA 6 (Scheme 110). ${ }^{141}$ This method enabled greener synthesis of halogenated/thiocyanated imidazoheterocycles 401 under solvent-free conditions. Reaction mechanism possibly involves in situ formation of [acetoxy(halo/thiocyanato)iodo]benzene from PIDA 6 and $\mathrm{M}-\mathrm{X}$, which serve as source of $\mathrm{X}^{+}$species facilitating electrophilic substitution on electron-rich substrates 400. Additionally, in situ bromination protocol was developed by utilizing $\mathrm{HBr}$ generated as by-product during the synthesis of fused $N$-heterocycles 402 and 404 from the condensation reaction of heterocyclic amine with bromoketone. The desired brominated products 403 and $\mathbf{4 0 5}$ are obtained in good yields. 

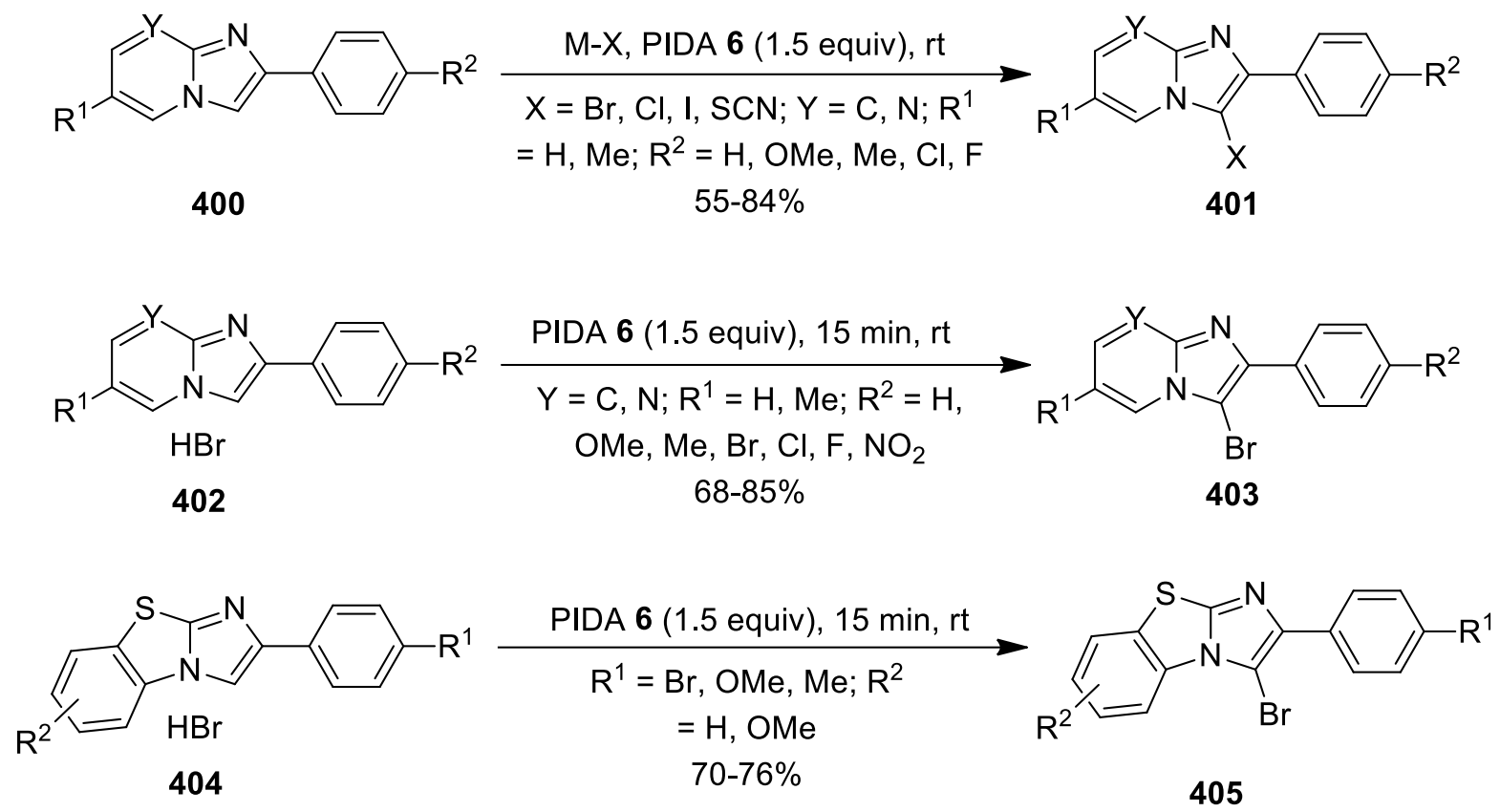

Scheme 110. PIDA-mediated C-3 halogenation/thiocyanation of fused N-heterocycles 400, 402 and 404.

In 2019, Sun and co-workers developed a regioselective C-2 cyanation of quinoline $\mathrm{N}$-oxides $\mathbf{3 7 5}$ by using trimethylsilyl cyanide 406 as an cyanating reagent with PIDA 6 as the oxidant (Scheme 111). ${ }^{142}$ Notably, PIDA activates the substrates and accelerates cleavage of $\mathrm{N}-\mathrm{O}$ bond. The present system showed remarkable compatibility for a wide range of substituents; particularly electron-rich substrates 375 produce 2cyanoquinolines $\mathbf{4 0 7}$ in better yields as compared to electron-deficient ones. Moreover, the scope of the reaction was extended towards pyridine $\mathrm{N}$-oxide and isoquinoline $\mathrm{N}$-oxide and desired products were obtained in useful yields.

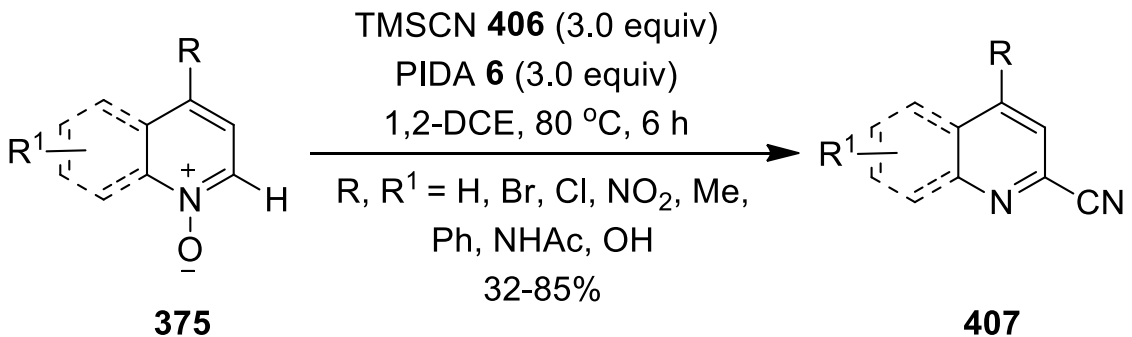

Scheme 111. PIDA-induced C-2 cyanation of quinoline N-oxides 375 by employing trimethylsilyl cyanide 406 as an cyanating reagent.

\subsection{Ring expansion of heterocycles}

In 2019, Murphy's research group realized fluorinative ring expansion of benzo-fused heterocycles $\mathbf{4 0 8}$ containing $\alpha$-exocyclic alkene using $p$-(difluoroiodo)toluene 19 as fluorinating reagent (Scheme 112). ${ }^{143}$ Anticipated ring expansion products $\mathbf{4 0 9}$ containing the $\beta, \beta$-difluoride moiety were isolated in valuable yields with shorter reaction time. Further fluorinative rearrangement of allene-based heterocycle 410 proceeded smoothly under similar conditions via 1,2-phenyl migration, to provide allylic gem-difluorides 411 in $29 \%$ yield. 
<smiles>[R]C1([R])CC(=C)c2ccccc2O1</smiles>

408

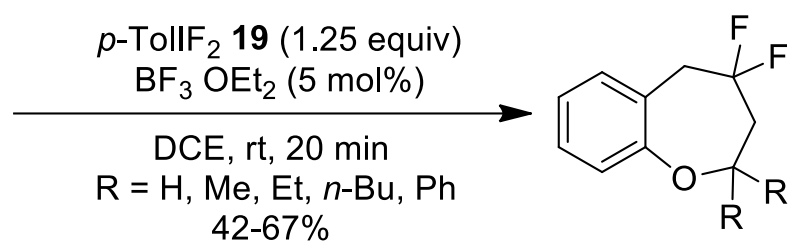

409

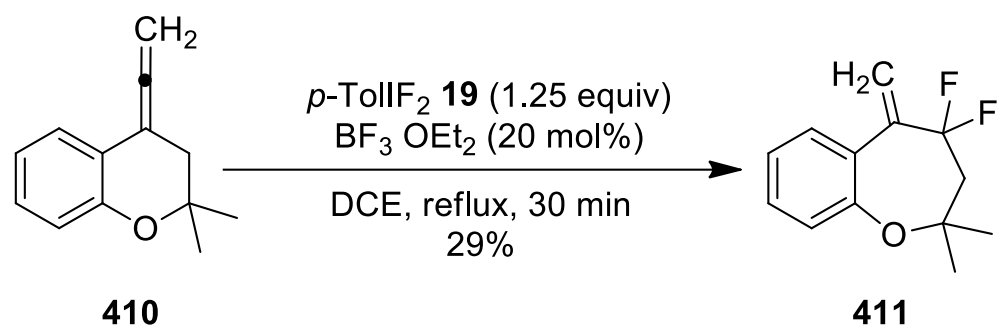

Scheme 112. Synthesis of benzo-fused heterocycles $\mathbf{4 0 9}$ and $\mathbf{4 1 1}$ through ring expansion of $\mathbf{4 0 8}$ and $\mathbf{4 1 0}$ using $p$-TollF 19 as fluorinating reagent.

\section{Conclusions}

Hypervalent iodine compounds are valuable reagents in organic synthesis due to their ready availability, easy handling, environment benign nature and low toxicity. Excellent electrophilic nature and versatile oxidizing ability of these reagents makes them promising alternate candidates for the heavy metal oxidants/catalysts. This review article summarizes the recent developments in the construction of heterocyclic scaffolds using hypervalent iodine reagents. Various stoichiometric or catalytic protocols have been developed to achieve synthesis of monocyclic, bicyclic, polycyclic and spirocyclic heterocycles under mild reaction conditions. More importantly, substantial work in the stereoselective synthesis of different heterocycles using chiral hypervalent reagents has been done with excellent enantioselectivities. Moreover, the application of hypervalent iodine reagents in the late-stage functionalization of heterocycles has been discussed briefly. Furthermore, development of new catalytic transformations that generates iodine(III) species in situ would be area of main focus in future. Apart from this, designing chiral hypervalent iodine-mediated enantioselective reactions still remains a great challenge because of the limited availability of chiral reagents, unsatisfactory enantioselectivity and limited substrate scope. Thus, development of novel asymmetric transformations promoted by chiral iodine(III) species provides an interesting field of research.

\section{Acknowledgements}

Fateh V. Singh is thankful to CSIR New Delhi [Grant No.: 02/(0330)/17-EMR-II] for the financial support. 


\section{References}

1. Yoshimura, A.; Zhdankin, V. V. Chem. Rev. 2016, 116, 3328.

https://doi.org/10.1021/acs.chemrev.5b00547

2. Silva, Jr.; L. F.; Olofsson, B. Nat. Prod. Rep. 2011, 28, 1722.

https://doi.org/10.1039/C1NP00028D

3. Yusubov, M. S.; Zhdankin, V. V. Curr. Org. Synth. 2012, 9, 247.

https://doi.org/10.2174/157017912799829021

4. Hypervalent lodine Chemistry: Modern Developments in Organic Synthesis, ed. T. Wirth, Topics in Current Chemistry, Springer-Verlag, 2016, vol. 373.

5. Patai's Chemistry of Functional Groups: The Chemistry of Hypervalent Halogen Compounds, ed. B. Olofsson, I. Marek and Z. Rappoport, Wiley, 2019.

6. Singh, F. V.; Wirth, T. Catalytic oxidation with hypervalent iodine In: Catalytic Oxidation in Organic Synthesis; Thieme; 2017, 1, 29. DOI 10.1055/sos-SD-225-23

7. Singh, F. V.; Wirth, T. Stereoselective reactions In Patai's Chemistry of Functional Groups, Eds. I.; John Wiley \& Sons, Ltd: Chichester, 2018.

8. Grelier, G.; Darses, B.; Dauban, P. Beilstein J. Org. Chem. 2018, 14, 1508. https://doi.org/10.3762/bjoc.14.128

9. Elsherbini, M.; Wirth, T. Chem. Eur. J. 2018, 24, 13399.

https://doi.org/10.1002/chem.201801232

10. Hyatt, I. F. D.; Dave, L.; David, N.; Kaur, K.; Medard, M.; Mowdawalla, C. Org. Biomol. Chem. 2019, 17, 7822.

https://doi.org/10.1039/C9OB01267B

11. Banerjee, S.; Bhoyare, V. W.; Patil, N. T. Chem. Commun. 2020, 56, 2677.

https://doi.org/10.1039/D0CC00106F

12. Tohma, H.; Kita, Y. Adv. Synth. Catal. 2004, 346, 111.

https://doi.org/10.1002/adsc.200303203

13. Mangaonkar, S. R.; Kole, P. B.; Singh, F. V. Synlett 2018, 18, 199.

https://doi.org/10.1055/s-0036-1588575

14. Zhdankin, V. V. ARKIVOC 2009, (i), 1.

https://doi.org/10.3998/ark.5550190.0010.101

15. Merritt, E. A.; Olofsson, B. Synthesis 2011, 4, 517.

https://doi.org/10.1055/s-0030-1258328

16. Dong, D.-Q.; Hao, S.-H.; Wang, Z.-L.; Chen, C. Org. Biomol. Chem. 2014, 12, 4278.

https://doi.org/10.1039/C4OB00318G

17. Singh, F.V.; Wirth, T. Synthesis, 2013, 45, 2499.

https://doi.org/10.1055/s-0033-1339679

18. Shetgaonkar, S. E.; Krishnan, M.; Singh, F. V. Mini Rev. Org. Chem. 2020, 17.

https://doi.org/10.2174/1570193X17999200727204349

19. Li, X.; Chen, P.; Liu, G. Beilstein J. Org. Chem. 2018, 14, 1813.

https://doi.org/10.3762/bjoc.14.154

20. Lee, J. H.; Choi, S.; Hong, K. B. Molecules 2019, 24, 2634.

https://doi.org/10.3390/molecules24142634 
21. Singh, F. V.; Wirth, T. Synthesis 2012, 44, 1171.

https://doi.org/10.1055/s-0031-1290588

22. Singh, F. V.; Wirth, T. Org. Lett. 2011, 13, 6504.

https://doi.org/10.1021/ol202800k

23. Singh, F. V.; Wirth, T. Chem. Asian J. 2014, 9, 950.

https://doi.org/10.1002/asia.201301582

24. Heterocycles in Natural Product Synthesis Editor(s): Prof. Krishna C. Majumdar Prof. Shital K.

Chattopadhyay

https://doi.org/10.1002/9783527634880

25. Martins, P.; Jesus, J.; Santos, S.; Raposo, L. R.; Roma-Rodrigues, C.; Baptista, P. V.; Fernandes, A. R.

Molecules 2015, 20, 16852 .

https://doi.org/10.3390/molecules200916852

26. Cabrele, C.; Reiser, O. J. Org. Chem. 2016, 81, 10109.

https://doi.org/10.1021/acs.joc.6b02034.

27. Smith, C. A.; Narouz, M. R.; Lummis, P. A.; Singh, I.; Nazemi, A.; Li, C.-H.; Crudden, C. M. Chem. Rev. 2019, $119,4986$.

https://doi.org/10.1021/acs.chemrev.8b00514

28. Gao, H.; Zhang, Q.; Shreeve, J. M. J. Mater. Chem. A 2020, 8, 4193.

https://doi.org/10.1039/C9TA12704F

29. Taylor, A. P.; Robinson, R. P.; Fobian, Y. M.; Blakemore, D. C.; Jones, L. H.; Fadeyi, O. Org. Biomol. Chem. 2016, 14, 6611.

https://doi.org/10.1039/C6OB00936K

30. Sun, J.; Zhang-Negrerie, D.; Du, Y.; Zhao, K. Rep. Org. Chem. 2016, 6, 25.

https://doi.org/10.2147/ROC.S84894

31. Kandimalla, S. R.; Parvathaneni, S. P.; Sabitha, G.; Reddy, B. V. S. Eur. J. Org. Chem. 2019, 1687. https://doi.org/10.1002/ejoc.201801469

32. Singh, F. V.; Kole, P. B.; Mangaonkar, S. R.; Shetgaonkar, S. E. Beilstein J. Org. Chem. 2018, 14, 1778. https://doi.org/10.3762/bjoc.14.152

33. Budhwan, R.; Yadav, S.; Murarka, S. Org. Biomol. Chem. 2019, 17, 6326.

https://doi.org/10.1039/С90B00694J

34. Mennie, K. M., Banik, S. M., Reichert, E. C.; Jacobsen, E. N. J. Am. Chem. Soc. 2018, 140, 4797.

https://doi.org/10.1021/jacs.8b02143

35. Glachet, T.; Marzag, H.; Rosa, N. S.; Colell, J. F. P.; Zhang, G.; Warren, W. S.; Franck, X.; Theis, T.; Reboul, V. J. Am. Chem. Soc. 2019, 141, 13689.

https://doi.org/10.1021/jacs.9b07035

36. Zhang, G.; Wang, Y.; Xu, J.; Sun, J.; Sun, F.; Zhang, Y.; Zhang, C.; Du, Y. Chem. Sci. 2020, $11,947$. https://doi.org/10.1039/c9sc05536c

37. Mangaonkar, S.; Singh, F. V. Synthesis 2019, 51, 4473.

https://doi.org/10.1055/s-0039-1690621

38. Singh, F. V.; Mangaonkar, S. R.; Kole, P. B. Synth. Commun. 2018, 48, 2169. https://doi.org/10.1080/00397911.2018.1479760

39. Asari, N., Takemoto, Y., Shinomoto, Y., Yagyu, T.; Yoshimura, A.; Zhdankin, V. V.; Saito, A. Asian J. Org. Chem. 2016, 5, 1314. 
https://doi.org/10.1002/ajoc.201600383

40. Okamura, Y.; Sato, D.; Yoshimura, A.; Zhdankin, V.V.; Saito, A. Adv. Synth. Catal. 2017, 359, 3243. http://dx.doi.org/10.1002/adsc.201700587

41. Yi, W., Liu, Q.-Y., Fang, X.-X., Lou, S.-C.; Liu, G.-Q. Org. Biomol. Chem. 2018, 16, 7012. https://doi.org/10.1039/C80B01474D

42. Yagyu, T., Takemoto, Y., Yoshimura, A., Zhdankin, V. V.; Saito, A. Org. Lett. 2017, 19, 2506. https://doi.org/10.1021/acs.orglett.7b00742

43. Xu, K.; Yang, S.; Ding, Z. Org. Chem. Front. 2020, 7, 69. https://doi.org/10.1039/c9qo01298b

44. Ranjith, J.; Rajesh, N.; Sridhar, B.; Krishna, P. R.; Org. Biomol. Chem. 2016, 14, 10074. https://doi.org/10.1039/c6ob01752e

45. Kamouka, S.; Moran, W. J. Beilstein J. Org. Chem. 2017, 13, 1823. https://doi.org/10.3762/bjoc.13.177

46. Alhalib, A.; Kamouka, S.; Moran, W. J. Org. Lett. 2015, 17, 1453. https://doi.org/10.1021/acs.orglett.5b00333

47. Liu, G.-Q.; Yang, C.-H.; Li, Y.-M. J. Org. Chem. 2015, 80, 11339. https://doi.org/10.1021/acs.joc.5b01832

48. Jeon, H.; Kima, D.; Lee, J. H.; Song, J.; Lee, W. S.; Kang, D. W.; Kang, S.; Lee, S. B.; Choi, S.; Hong, K. B. Adv. Synth. Catal. 2018, 360, 779. https://doi.org/10.1002/adsc.201701087

49. Scheidt, F.; Thiehoff, C.; Yilmaz, G.; Meyer, S.; Daniliuc, C. G.; Kehr, G.; Gilmour, R. Beilstein J. Org. Chem. 2018, 14, 1021.

https://doi.org/10.3762/bjoc.14.88

50. Carlucci, C.; Tota, A.; Colella, M.; Ronamazzi, G.; Clarkson, G. J.; Luisi, R.; Degennaro, L. Chem. Heterocycl. Com. 2018, 54, 428.

https://doi.org/10.1007/s10593-018-2288-9

51. Singhal, A.; Parumala, S. K. R.; Sharma, A.; Peddinti, R. K. Tetrahedron Lett. 2016, 57, 719. http://dx.doi.org/10.1016/i.tetlet.2015.10.038

52. Kobayashi, E.; Togo, H. Synthesis 2019, 51, 3723. http://dx.doi.org/10.1055/s-0039-1690102.

53. Waheed, M., Ahmed, N., Alsharif, M. A.; Alahmdi, M. I.; Mukhtar, S. ChemistrySelect 2019, 4, 1872. https://doi.org/10.1002/slct.201803927

54. Park, S. W.; Kim, S.-H.; Song, J.; Park, G. Y.; Kim, D.; Nam, T.-G.; Hong, K. B. Beilstein J. Org. Chem. 2018, 14, 1028.

https://doi.org/10.3762/bjoc.14.89

55. Yu, J.-M.; Cai, C. Org. Biomol. Chem. 2018, 16, 490.

https://doi.org/10.1039/C70B02892J

56. Yoshimura, A.; Nguyen, K. C.; Klasen, S. C.; Postnikov, P. S.; Yusubov, M. S.; Saito, A.; Nemykin, V. N.; Zhdankin, V. V. Asian J. Org. Chem. 2016, 5, 1128.

http://dx.doi.org/10.1002/ajoc.201600247

57. Yoshimura, A.; Nguyen, K. C.; Klasen, S. C.; Saito, A.; Nemykina, V. N.; Zhdankin, V. V. Chem. Commun. 2015, 51, 7835.

http://dx.doi.org/10.1039/c5cc02009c 
58. Chauhan, J.; Ravva, M. K.; Sen, S. Org. Lett. 2019, 21, 6562.

http://dx.doi.org/10.1021/acs.orglett.9b02542

59. Liu, B.; Shi, B.-F. Synlett 2016, 27, 2396.

http://dx.doi.org/10.1055/s-0035-1562508

60. Yakura, T.; Horiuchi, Y.; Nishimura, Y.; Yamada, A.; Nambu, H.; Fujiwara, T. Adv. Synth. Catal. 2016, 358, 869.

http://dx.doi.org/10.1002/adsc.201500795

61. Yakura, T.; Fujiwara, T.; Nishi, H.; Nishimura, Y.; Nambu, H. Synlett 2018, 29, 2316. http://dx.doi.org/10.1055/s-0037-1610657

62. Gelis, C., Dumoulin, A., Bekkaye, M., Neuville, L.; Masson, G. Org. Lett. 2017, 19, 278. http://dx.doi.org/10.1021/acs.orglett.6b03631

63. S.; Alazet, Vaillant, F. L.; Nicolai, S.; Courant, T.; Waser, J. Chem. Eur. J. 2017, $23,9501$. http://dx.doi.org/10.1002/chem.201702599

64. Kiyokawa, K.; Ito, R.; Takemoto, K.; Minakata, S. Chem. Commun. 2018, 54, 7609. http://dx.doi.org/10.1039/c8cc03735c

65. Kiyokawa, K.; Takemoto, K.; Yahata, S.; Kojima, T.; Minakata, S. Synthesis 2017, 49, 2907. http://dx.doi.org/10.1055/s-0036-1588987

66. Mizar, P.; Niebuhr, R.; Hutchings, M.; Farooq, U.; Wirth, T. Chem. Eur. J. 2016, 22, 1614. http://dx.doi.org/10.1002/chem.201504636

67. Kitamura, T.; Miyake, A.; Muta, K.; Oyamada, J. J. Org. Chem. 2017, 82, 11721. http://dx.doi.org/10.1021/acs.joc.7b01266

68. Huang, H.; Yang, Q.; Zhang, Q.; Wu, J.; Liu, Y.; Song, C.; Chang, J. Adv. Synth. Catal. 2016, 358, 1130. http://dx.doi.org/10.1002/adsc.201501071

69. Xu, H.; Liu, H.-W.; Chen, K.; Wang, G.-W. J. Org. Chem. 2018, 83, 6035. http://dx.doi.org/10.1021/acs.joc.8b00665.

70. Chen, H.; Kaga, A.; Chiba, S. Org. Biomol. Chem. 2016, 14, 5481. https://doi.org/10.1039/C5OB01854D

71. Ma, H.; Zhang, X.; Chen, L.; Yu, W. J. Org. Chem. 2017, 82, 11841. https://doi.org/10.1021/acs.joc.7b01361

72. Shen, K.; Wang, Q. Org. Chem. Front. 2016, 3, 222. https://doi.org/10.1039/C5Q000353A

73. Shen, K.; Wang, Q. J. Am. Chem. Soc. 2017, 139, 13110. https://doi.org/10.1021/jacs.7b06852

74. T.; Borelli, S.; Brenna, G.; Broggini, J.; Oble, Poli, G., Adv. Synth. Catal. 2017, 359, 623. https://doi.org/10.1002/adsc.201600813

75. Han, Y.; Sun, Y.; Abdukader, A.; Liu, B.; Wang, D. Catal. Lett. 2018, 148, 3486. https://doi.org/10.1007/s10562-018-2541-y

76. Broggini, G.; Beccalli, E. M.; Borelli, T.; Brusa, F.; Gazzola, S.; Mazza, A. Eur. J. Org. Chem. 2015, 4261. https://doi.org/10.1002/ejoc.201500386

77. Wang, H.; Frings, M.; Bolm, C. Org. Lett. 2016, 18, 2431. https://doi.org/10.1021/acs.orglett.6b00958

78. Walters, J. C.; Tierno, A. F.; Dubin, A. H.; Wengryniuk, S. E. Eur. J. Org. Chem. 2018, 1460. http://dx.doi.org/10.1002/ejoc.201800118 
79. McNally, A.; Haffemayer, B.; Collins, B. S. L.; Gaunt, M. J. Nature, 2014, 510, 129.

http://dx.doi.org/10.1038/nature13389

80. Nappi, M.; He, C.; Whitehurst, W. G.; Chappell, B. G. N.; Gaunt, M. J. Angew. Chem. 2018, 130, 3232. http://dx.doi.org/10.1002/anie.201800519

81. Tao, J.; Estrada, C. D.; Murphy, G. K. Chem. Commun. 2017, 53, 9004. https://doi.org/10.1039/C7CC04859A

82. Yoshimura, A.; Nguyen, K. C.; Rohde, G. T.; Saito, A.; Yusubov, M. S.; Zhdankin, V. V. Adv. Synth. Catal. 2016, 358, 2340.

http://dx.doi.org/10.1002/adsc.201600331

83. Yoshimura, A.; Nguyen, K. C.; Rohde, G. T.; Postnikov, P. S.; Yusubov, M. S.; Zhdankin, V. V. J. Org. Chem. 2017, 82, 11742.

http://dx.doi.org/10.1021/acs.joc.7b01462

84. Xu, T.; Wang, D.; Tong, X. Org. Lett. 2019, 21, 5368.

http://dx.doi.org/10.1021/acs.orglett.9b02096

85. Bedford, R. B.; J. G.; Bowen, Méndez-Gálvez, C. J. Org. Chem. 2017, 82, 1719. http://dx.doi.org/10.1021/acs.joc.6b02970

86. Li, H.; Li, X.; Yu, Y.; Li, J.; Liu, Y.; Li, H.; Wang, W. Org. Lett. 2017, 19, 2010. http://dx.doi.org/10.1021/acs.orglett.7b00566

87. Nakamura, A.; Tanaka, S.; Imamiya, A.; Takane, R.; Ohta, C.; Fujimura, K.; Maegawa, T.; Miki, Y. Org. Biomol. Chem. 2017, 15, 6702. https://doi.org/10.1039/C70B01536D

88. Xia, H.-D.; Zhang, Y.-D.; Wang, Y.-H.; Zhang, C. Org. Lett. 2018, 20, 4052. https://doi.org/10.1021/acs.orglett.8b01615

89. Bose, A.; Maiti, S.; Sau, S.; Mal, P. Chem. Commun. 2019, 55, 2066. https://doi.org/10.1039/c8cc09100e

90. Bose, A., Sau, S.; Mal, P. Eur. J. Org. Chem. 2019, 4105. https://doi.org/10.1002/ejoc.201900732

91. Singh, F. V.; Mangaonkar, S. R. Synthesis 2018, 50, 4940. https://doi.org/10.1055/s-0037-1610650

92. Mangaonkar, S. R.; Shetgaonkar, S. E.; Vernekar, A. A.; Singh. F. V. ChemistrySelect 2020, 5, 10754. https://doi.org/10.1002/slct.202002860

93. Zhang, H.; Shen, J.; Yang, Z.; Cui, X. RSC Adv. 2019, 9, 7718. https://doi.org/10.1039/c9ra01200a

94. Chen, T.; Chen, X.; Wei, J.; Lin, D.; Xie, Y.; Zeng, W. Org. Lett. 2016, 18, 2078. https://doi.org/10.1021/acs.orglett.6b00709

95. Ding, Q., He, H.; Cai, Q. Org. Lett. 2018, 20, 4554. https://doi.org/10.1021/acs.orglett.8b01849

96. Fan, H.; Pan, P.; Zhang, Y.; Wang, W. Org. Lett. 2018, 20, 7929. https://doi.org/10.1021/acs.orglett.8b03503

97. Woerly, E. M.; Banik, S. M.; Jacobsen, E. N. J. Am. Chem. Soc. 2016, 138, 13858. https://doi.org/10.1021/jacs.6b09499

98. Möckel, R., Babaoglu, E.; Hilt, G. Chem. Eur. J. 2018, 24, 15781. https://doi.org/10.1002/chem.201804152 
99. Zhang, H.; Shen, J.; Cheng, G.; Feng, Y.; Cui, X. Org. Lett. 2018, 20, 664.

https://doi.org/10.1021/acs.orglett.7b03804

100. Kelley, B. T.; Walters, J. C.; Wengryniuk, S. E. Org. Lett. 2016, 18, 1896.

https://doi.org/10.1021/acs.orglett.6b00672

101. Yamakoshi, W.; Arisawa, M.; Murai, K. Org. Lett. 2019, 21, 3023.

https://doi.org/10.1021/acs.orglett.9b00559

102. Maiti, S.; Mal, P. Org. Lett. 2017, 19, 2454.

https://doi.org/10.1021/10.1021/acs.orglett.7b01117

103. Maiti, S., Achar, T. K.; Mal, P. Org. Lett. 2017, 19, 2006.

https://doi.org/10.1021/acs.orglett.7b00562

104. Bal, A., Maiti, S., Mal, P. J. Org. Chem. 2018, 83, 11278.

https://doi.org/10.1021/acs.joc.8b01857

105. Murai, K.; Kobayashi, T.; Miyoshi, M.; Fujioka, H. Org. Lett. 2018, 20, 2333.

https://doi.org/10.1021/acs.joc.8b01857

106. Shimogaki, M.; Fujita, M.; Sugimura, T. Angew. Chem. Int. Ed. 2016, 55, 15797.

http://dx.doi.org/10.1002/anie.201609110

107. Waghmode, N. A.; Kalbandhe, A. H.; Thorat, P. B.; Karade N. N.; Tetrahedron Lett. 2016, 57, 680.

https://doi.org/10.1016/i.tetlet.2015.12.117

108. Hong, F.; Lu, N.; Lu, B.; Cheng, J. Adv. Synth. Catal. 2017, 359, 3299.

https://doi.org/10.1002/adsc.201700761

109. Chen, X.-Y.; Zhang, C.-Shen.; Yi, L.; Gao, Z.-H.; Wang, Z.-X.; Ye, S. CCS Chem. 2019, 1, 343.

http://dx.doi.org/10.31635/ccschem.019.20190020

110. Deng, Q.; Xia, W.; Hussain, M. I.; Zhang, X.; Hu, W.; Xiong, Y. J. Org. Chem. 2020, 85, 3125. https://dx.doi.org/10.1021/acs.joc.9b03012

111. Zhang, D.-Y.; Xu, L.; Wu, H.; Gong, L.-Z. Chem. Eur. J. 2015, 21, 10314.

http://dx.doi.org/10.1002/chem.201501583

112. Laevens, B. A.; Tao, J.; Murphy, G. K. J. Org. Chem. 2017, 82, 11903.

http://dx.doi.org/10.1021/acs.joc.7b01639

113. Uyanik, M.; Sasakura, N.; Mizuno, M.; Ishihara, K. ACS Catal. 2017, 7, 872.

https://doi.org/10.1021/acscatal.6b03380

114. Uyanik, M., Yasui, T.; Ishihara, K. J. Org. Chem. 2017, 82, 11946.

https://doi.org/10.1021/acs.joc.7b01941

115. Hempel, C., Maichle-mössmer, C.; Pericas, M. A.; Nachtsheim, B. J. Adv. Synth. Catal. 2017, $359,2931$. http://dx.doi.org/10.1002/adsc.201700246

116. Abazid, A. H.; Nachtsheim, B. J. Angew. Chem. Int. Ed. 2020, 59, 1479.

https://doi.org/10.1002/anie.201912023

117. Ogasawara, M.; Sasa, H.; Hu, H.; Amano, Y.; Nakajima, H.; Takenaga, N.; Nakajima, K.; Kita, Y.; Takahashi, T.; Dohi, T. Org. Lett. 2017, 19, 4102.

https://doi.org/10.1021/acs.orglett.7b01876

118. Imrich, M. R.; Ziegler, T. Tetrahedron Lett. 2019, 60, 150954.

https://doi.org/10.1016/i.tetlet.2019.150954

119. Antien, A. K., Pouysegu, L., Deffieux, D., Peixoto, P. A.; Quideau, S. Chem. Eur. J. 2019, 25, 2852.

https://doi.org/10.1002/chem.201805761 
120. Zhao, G.-H.; Li, B.-Q.; Wang, S.-S.; Liu, M.; Chen, Y.; Wang, B. J. Org. Chem. 2020, 85, 14, 9367. https://doi.org/10.1021/acs.joc.0c00971

121. Xing, Q.; Liang, H.; Bao, M.; Li, X.; Zhang, J.; Bi, T.; Zhang, Y.; Xu, J.; Du, Y.; Zhao, K. Adv. Synth. Catal. 2019, 361, 4669. https://doi.org/10.1002/adsc.201900652

122. Jain, N., Xu, S.; Ciufolini, M. A. Chem. A Eur. J. 2017, 23, 4542. https://doi.org/10.1002/chem.201700667

123. Deng, R.; Zhan, S.; Li, C.; Gu, Z. Angew. Chem. 2020, 59, 3093. https://doi.org/10.1002/ange.201913373

124. Tariq, M. U.; Moran, W. J. Eur. J. Org. Chem. 2020, 5153. https://doi.org/10.1002/ejoc.202000840

125. Odagi, M.; Okuda, K.; Ishizuka, H.; Adachi, K.; Nagasawa, K. Asian J. Org. Chem 2020, 9, 218. https://doi.org/10.1002/ajoc.201900726

126. Mondal, S.; Samanta, S.; Jana, S.; Hajra, A. J. Org. Chem. 2017, 82, 4504. https://doi.org/10.1021/acs.joc.7b00564

127. Sun, B.; Yan, Z.; Jin, C.; Su, W. Synlett 2018, 29, 2432. https://doi.org/10.1055/s-0037-1610293

128. Lu, N. ; Huang, L.; Xie, L.; Cheng, J. Eur. J. Org. Chem. 2018, 3437. http://dx.doi.org/10.1002/ejoc.201800416

129. Chen, W.-T.; Gao, L.-H.; Bao, W.-H.; Wei, W.-T. J. Org. Chem. 2018, 83, 11074. https://doi.org/10.1021/acs.joc.8b01678

130. Xu, J.; Qiao, L.; Ying, B.; Zhu, Xi.; C. Shen, Zhang, P. Org. Chem. Front. 2017, 4, 1116. https://doi.org/10.1039/c6qo00655h

131. Sakamoto, R.; Kashiwagi, H.; Maruoka, K. Org. Lett. 2017, 19, 5126. https://doi.org/10.1021/acs.orglett.7b02416

132. Sen, C.; Ghosh, S. C. Adv. Synth. Catal. 2018, 360, 905. https://doi.org/10.1002/adsc.201701330

133. Genovino, J.; Lian, Y.; Zhang, Y.; Hope, T. O.; Juneau, A.; Gagne', Y.; Ingle, G.; Frenette, M. Org. Lett. 2018, $20,3229$.

https://doi.org/10.1021/acs.orglett.8b01085

134. Zhang, X.-Y.; Weng, W.-Z.; Liang, H.; Yang, H.; Zhang, B. Org. Lett. 2018, 20, 4686. https://doi.org/10.1021/acs.orglett.8b02016

135. Li, G.-X.; Hu, X.; He G.; Chen, G. Chem. Sci. 2019, 10, 688. https://doi.org/10.1039/c8sc04134b

136. Roy, A.; Das, M. K.; Chaudhuri, S.; Bisai, A. J. Org. Chem. 2018, 83, 403. https://doi.org/10.1021/acs.joc.7b02797

137. Kotagiri, R.; Adepu, R. Eur. J. Org. Chem. 2018, 4556. https://doi.org/10.1002/ejoc.201800723

138. De, A.; Santra, S.; Hajra, A.; Zyryanov, G. V.; Majee, A. J. Org. Chem. 2019, 84, 11735. http://dx.doi.org/110.1021/acs.joc.9b01625

139. Patel, O. P. S.; Jaspal, S.; Shinde, V. N.; Nandwana, N. K.; Rangan, K.; Kumar A. J. Org. Chem. 2020, 85, 7309.

https://dx.doi.org/10.1021/acs.joc.0c00674 
140. Himabindu, V.; Parvathaneni, S. P.; Rao, V. J. New J. Chem. 2018, 42, 18889.

https://dx.doi.org/10.1039/c8nj03822h

141. Indukuri, D. R.; Potuganti, G. R.; Alla, M. Synlett 2019, 30, 1573.

https://dx.doi.org/10.1055/s-0037-1611856

142. Xua, F.; Li, Y.; Huang, X.; Fang, X.; Li, Z.; Jiang, H.; Qiao, J.; Chu, W.; Sun, Z. Adv. Synth. Catal. 2019, 361, 520.

https://doi.org/10.1002/adsc.201801185

143. Zhao, Z.; To, A. J.; Murphy, G. K. Chem. Commun. 2019, 55, 14821.

https://doi.org/10.1039/C9CC08310C

\section{Authors' Biographies}

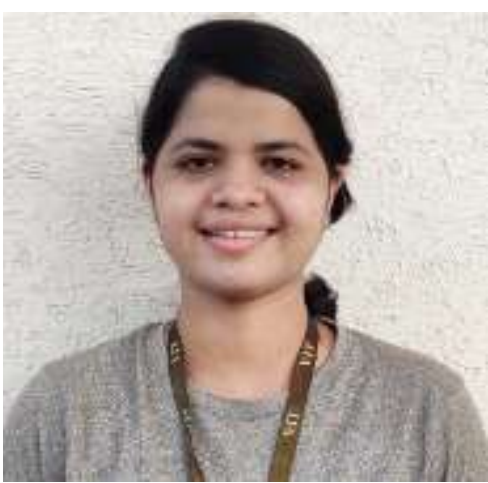

Samata E. Shetgaonkar was born in Morjim, Pernem, Goa, India in 1992. After completing her M.Sc. in Organic Chemistry from Goa University, Goa, India, in 2015, she is pursuing her PhD degree under the supervision of Dr. Fateh V Singh in the field of Hypervalent lodine Chemistry at VIT Chennai, Tamil Nadu, India. During her doctoral studies, she is involved in the synthesis of novel hypervalent reagents and their application in organic synthesis including asymmetric synthesis.

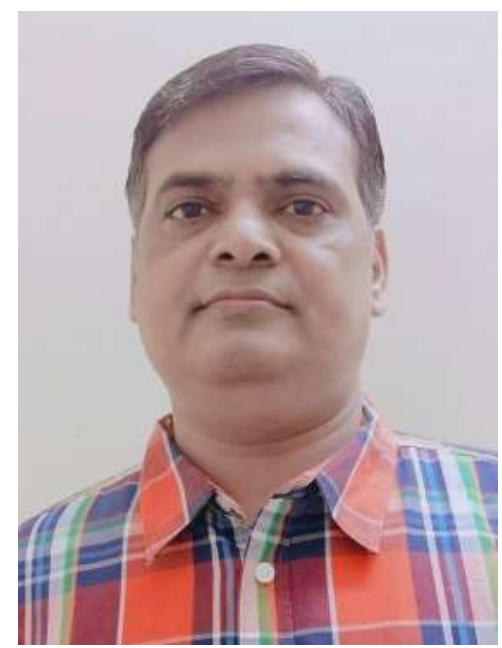

Fateh V Singh was born in Ravani Katiry, Bulandshahr, UP, India in 1976. He has completed his MSc in Chemistry from SSV College, Hapur, UP, India in 1998. He has persued his PhD in 2007 with Dr Atul Goel (CSIR-CDRI, Lucknow, India). After the completion of his doctoral studies, he started his first postdoctoral studies (FAPESP fellowship) with Prof. H A Stefani at USP, São Paulo, Brazil and worked with him for more than two years in the 
area organotrifluoroborate chemistry. In 2010, he joined as Marie Curie postdoctoral fellow with Prof. Thomas Wirth at Cardiff University, UK and worked two years in the area of organoselenium and hypervalent iodine chemistry. He received Dr D S Kothari fellowship in 2013 and worked with Prof. G Mugesh at IISc Bangalore, India for a short stay. In 2014, he started his independent career and joined VIT University, Chennai as an Assistant Professor. Mainly, his research group is interested in the findings of new organoselenium and hypervalent catalysts for organic synthesis. Moreover, his research group is also involved in the development of new organic fluorescent molecules for OLEDs and chemical sensors. Currently, he is having different research grants from Government of India. He has already published more than 50 research papers, several book chapters and review articles.

This paper is an open access article distributed under the terms of the Creative Commons Attribution (CC BY) license (http://creativecommons.org/licenses/by/4.0/) 